\title{
Improved Holographic QCD
}

\author{
Umut Gürsoy ${ }^{1}$, Elias Kiritsis ${ }^{2 *}$ Liuba Mazzanti $^{3}$, Georgios Michalogiorgakis ${ }^{4}$ and \\ Francesco $\mathrm{Nitti}^{5}$ \\ 1 Institute for Theoretical Physics, Utrecht University; Leuvenlaan 4, 3584 CE Utrecht, \\ The Netherlands. \\ 2 Crete Center for Theoretical Physics, Department of Physics, University of Crete \\ 71003 Heraklion, Greece
}

3 Departamento de Física de Partículas, Universidade de Santiago de Composteld

and

Instituto Galego de Física de Altas Enerxías (IGFAE)

E-15782, Santiago de Compostela, Spain

${ }^{4}$ Department of Physics, Purdue University, 525 Northwestern Avenue, West Lafayette, IN 47907-2036

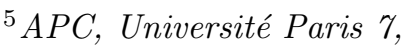

Bâtiment Condorcet, F-75205, Paris Cedex 13, France

(UMR du CNRS 7164).

ABSTRACT: We provide a review to holographic models based on Einstein-dilaton gravity with a potential in 5 dimensions. Such theories, for a judicious choice of potential are very close to the physics of large-N YM theory both at zero and finite temperature. The zero temperature glueball spectra as well as their finite temperature thermodynamic functions compare well with lattice data. The model can be used to calculate transport coefficients, like bulk viscosity, the drag force and jet quenching parameters, relevant for the physics of the Quark-Gluon Plasma.

KeYwords: Gauge-gravity correspondence, QCD, Quark Gluon Plasma, Holographid Gauge theory

Based on lectures given at the 5th Aegean Summer School (Milos, Greece), September 2009. To appear in the proceedings.

\footnotetext{
*On leave of absence from APC, Université Paris 7, (UMR du CNRS 7164).
} 


\section{Contents}

1. Introduction 2

2. The 5D model 7

3. Scheme dependence

4. The potential and the parameters of the model 13

5. Matching the thermodynamics of large- $N_{c}$ YM

5.1 Latent heat and equation of state 19

5.2 Glueball spectrum 21

5.3 Critical temperature 23

5.4 String tension 24

5.5 CP-odd sector 25

5.6 Coupling normalization 27

6. Bulk viscosity 27

6.1 The holographic computation 29

6.2 Holographic explanation for the rise of $\zeta / s$ near $T_{c}$ and the small black-hole branch 32

6.3 The adiabatic approximation 35

6.4 Buchel's bound 37

7. The drag force on strings and heavy quarks 39

7.1 The drag force 40

7.2 The relativistic asymptotics 42

7.3 The non-relativistic asymptotics

7.4 The diffusion time

7.5 Including the medium-induced correction to the quark mass 44

7.6 Temperature matching and diffusion time estimates

8. Jet quenching parameter 48

9. Discussion and outlook 54

Acknowledgements 58

References $\quad 59$ 


\section{Introduction}

The experimental efforts at RHIC, [1] have provided a novel window in the physics of the strong interactions. The consensus on the existing data is that shortly after the collision, a ball of quark-gluon plasma (QGP) forms that is at thermal equilibrium, and subsequently expands until its temperature falls below the QCD transition (or crossover) where it finally hadronizes. Relativistic hydrodynamics describes very well the QGP [2], with a shearviscosity to entropy density ratio close to that of $\mathcal{N}=4$ SYM, [3]. The QGP is at strong coupling, and it necessitates a treatment beyond perturbative QCD approaches, 幽. Moreover, although the shear viscosity from $\mathcal{N}=4$ seems to be close to that "measured" by experiment, lattice data indicate that in the relevant RHIC range $1 \leq \frac{T}{T_{c}} \leq 3$ the QGP seems not to be a fully conformal fluid. Therefore the bulk viscosity may play a role near the phase transition [5, 6]. The lattice techniques have been successfully used to study the thermal behavior of QCD, however they are not easily extended to the computation of hydrodynamic quantities. They can be used however, together with parametrizations of correlators in order to pin down parameters [6]. On the other hand, approaches based on holography have the potential to address directly the real-time strong coupling physics relevant for experiment.

In the bottom-up holographic model of AdS/QCD [7], the bulk viscosity is zero as conformal invariance is essentially not broken (the stress tensor is traceless). In the soft-wall model [8], no reliable calculation can be done for glue correlators and therefore transport coefficients are ill-defined. Similar remarks hold for other phenomenologically interesting observables as the drag force and the jet quenching parameter [9, 10, 11, 12].

Top-down holographic models of QCD displaying all relevant features of the theory have been difficult to obtain. Bottom-up models based on AdS slices [13] have given some insights mostly in the meson sector, [7] but necessarily lack many important holographic features of QCD. A hybrid approach has been advocated [14, 15] combining features of bottom-up and top-down models. An similar approach was proposed independently in [16]. Such an approach, called Improved Holographic QCD (or IHQCD for short) is essentially a five-dimensional dilaton-gravity system with a non-trivial dilaton potential. Flavor can be eventually added in the form of $N_{f}$ space-time filling $D 4-\overline{D 4}$ brane pairs, supporting $U\left(N_{f}\right)_{L} \times U\left(N_{f}\right)_{R}$ gauge fields and a bi-fundamental scalar 17. The UV asymptotics of the potential are fixed by QCD perturbation theory, while the IR asymptotics of the potential can be fixed by confinement and linear glueball asymptotics. An analysis of the

finite temperature behavior [18, 19] has shown that the phase structure is exactly what one would expect from YM. A potential with a single free parameter tuned to match the zero temperature glueball spectrum was able to agree with the thermodynamic behavior of glue to a good degree, 18]. Similar results, but with somewhat different potentials were also obtained in [16, 20]

In 18, 19, 16] it was shown that Einstein-dilaton gravity with a strictly monotonic dilaton potential that grows sufficiently fast, generically shares the same phase structure and thermodynamics of finite-temperature pure Yang-Mills theory at large $N_{c}$. There is a deconfinement phase transition (dual to a Hawking-Page phase transition between a black 
hole and thermal gas background on the gravity side), which is generically first order. The latent heat scales as $N_{c}^{2}$. In the deconfined gluon-plasma phase, the free energy slowly approaches that of a free gluon gas at high temperature, and the speed of sound starts from a small value at $T_{c}$ and approaches the conformal value $c_{s}^{2}=1 / 3$ as the temperature increases. The deviation from conformal invariance is strongest at $T_{c}$, and is signaled by the presence of a non-trivial gluon condensate, which on the gravity side emerges as a deviation of the scalar solution that behaves asymptotically as $r^{4}$ close to the UV boundary. In the CP-violating sector, the topological vacuum density $\operatorname{Tr} F \tilde{F}$ has zero expectation value in the deconfined phase, in agreement with lattice results [21] and large- $N_{c}$ expectations.

The analysis performed in [19] was completely general and did not rely on any specific form of the dilaton potential $V(\lambda)$. A detailed analysis of an explicit model in [22] shows that the thermodynamics matches quantitatively the thermodynamics of pure Yang-Mills theory. The (dimensionless) free energy, entropy density, latent heat and speed of sound, obtained on the gravity side by numerical integration of the $5 \mathrm{D}$ field equations, can be compared with the corresponding quantities, calculated on the lattice for pure Yang-Mills at finite- $T$, resulting in excellent agreement, for the temperature range that is accessible by lattice techniques. The same model also shows a good agreement with the lattice calculation of glueball mass ratios at zero temperature, and the value of the deconfining critical temperature (in units of the lowest glueball mass) is also in good agreement with the lattice results.

In short, the model we present gives a good phenomenological holographic description of most static properties ${ }^{1}$ (spectrum and equilibrium thermodynamics) of large- $N_{c}$ pure Yang-Mills, as computed on the lattice, for energies up to several times $T_{c}$. Thus it constitutes a good starting point for the computation of dynamical observables in a realistic holographic dual to QCD (as opposed to e.g. $\mathcal{N}=4$ SYM), such as transport coefficients and other hydrodynamic properties that are not easily accessible by lattice techniques, at energies and temperatures relevant for relativistic heavy-ion collision experiments. We will report on such a calculation in the near future.

The vacuum solution in this model is described in terms of two basic bulk fields, the metric and the dilaton. These are not the only bulk fields however, as the bulk theory is expected to have an a priori infinite number of fields, dual to all possible YM operators. In particular we know from the string theory side that there are a few other low mass fields, namely the RR axion (dual to the QCD $\theta$-angle) the NSNS and RR two forms $B_{2}$ and $C_{2}$ as well as other higher-level fields. With the exception of the RR axion, such fields are dual to higher-dimension and/or higher-spin operators of YM. Again, with the exception of the $\mathrm{RR}$ axion, they are not expected to play an important role into the structure of the vacuum and this is why we neglect them when we solve the equations of motion. However, they are going to generate several new towers of glueball states beyond those that we discuss in this paper (namely the $0^{++}$glueballs associated to dilaton fluctuations, $2^{++}$glueballs associated to graviton fluctuations and $0^{-+}$glueballs associated to RR axion fluctuations). Such fields can be included in the effective action and the associated glueball spectra

\footnotetext{
${ }^{1}$ There are very few observables also that are not in agreement with YM. They are discussed in detail in 115 .
} 
calculated. Since we do not know the detailed structure of the associated string theory, their effective action will depend on more semi-phenomenological functions like $Z(\lambda)$ in (5.9). These functions can again be determined in a way similar to $Z(\lambda)$. In particular including the $B_{2}$ and $C_{2}$ field will provide $1^{+-}$glueballs among others. Fields with spin greater than 2 are necessarily stringy in origin. We will not deal further with extra fields, like $B_{2}$ and $C_{2}$ and other as they are not particularly relevant for the purposes of this model, namely the study of finite temperature physics in the deconfined case. We will only consider the axion, as its physics is related to the CP-odd sector of YM with an obvious phenomenological importance.

It is well documented that string theory duals of YM must have strong curvatures in the UV regime. This has been explained in detail in [15] where it was also argued, that although the asymptotic AdS boundary geometry is due to the curvature non-linearities of the associated string theory, the inwards geometry is perturbative around AdS, with logarithmic corrections, generating the YM perturbation theory. The present model is constructed so that it takes the asymptotic AdS geometry for granted, by introducing the associated vacuum energy by hand, and simulates the perturbative YM expansion by an appropriate dilaton potential. In the IR, we do not expect strong curvatures in the string frame, and indeed the preferred backgrounds have this property. In this sense the model contains in itself the relevant expected effects that should arise from strong curvatures in all regimes. These issues have been explained in [14] and in more detail in [15].

A different and interesting direction is the use of such models to study the expansion of the plasma and the associated dynamics. Such a context is similar to what happens on cosmology, especially the one related to the Randall-Sundrum setup. Indeed in this case the expansion can be found by following the geodesic motion of probe branes in the relevant background, [23]-[26]. This generalizes to more complicated backgrounds, [26], like the ones studied here.

Once we have a holographic model we trust, we should calculate observables, like transport coefficients that are hard to calculate on the lattice. A first class of transport coefficients are viscosity coefficients. ${ }^{2}$ A general fluid is characterized by two viscosity coefficients, the shear $\eta$ and the bulk viscosity $\zeta$. The shear viscosity in strongly coupled theories described by gravity duals was shown to be universal, [3]. In particular, the ratio $\eta / s$, with $s$ the entropy density, is equal to $\frac{1}{4 \pi}$. This is correlated to the universality of lowenergy scattering of gravitons from black holes. It is also known that deviations from this value can only be generated by higher curvature terms that contain the Riemann tensor (as opposed to the Ricci tensor of the scalar curvature). In QCD, as the theory is strongly coupled in the temperature range $T_{c} \leq T \leq 3 T_{c}$, we would expect that $\eta / s \simeq \frac{1}{4 \pi}$. Recent lattice calculations, [28] agree with this expectations although potential systematic errors in lattice calculations of transport coefficients can be large.

Conformal invariance forces the bulk viscosity to vanish. Therefore the $\mathcal{N}=4 \mathrm{SYM}$ plasma, being a conformal fluid, has vanishing bulk viscosity. QCD on the other hand is not

\footnotetext{
${ }^{2}$ These are the leading transport coefficients in the derivative expansion. There are subleading coefficients that have been calculated recently for $\mathcal{N}=4 \mathrm{SYM}$, 27]. However, at the present level of accuracy, they cannot affect substantially the comparison to experimental data, [2].
} 
a conformal theory. The classical theory is however conformally invariant and asymptotic freedom implies that conformal invariance is a good approximation in the UV. This would suggest that the bulk viscosity to entropy ratio is negligible at large temperatures. However it is not expected to be so in the IR: as mentioned earlier lattice data indicate that in the relevant RHIC range $1 \leq \frac{T}{T_{c}} \leq 3$ the QGP seems not to be a fully conformal fluid. Therefore the bulk viscosity may play a role near the phase transition.

So far there have been two approaches that have calculated the bulk viscosity in YM/QCD, 229, 30, 31, 32 and have both indicated that the bulk viscosity rises near the phase transition as naive expectation would suggest. The first used the method of sum rules in conjunction with input from Lattice thermodynamics, [29, 30, 31]. It suggested a dramatic rise of the bulk viscosity near $T_{c}$ although the absolute normalization of the result is uncertain. The reason is that this method relies on an ansatz for the density associated with stress-tensor two point functions that are otherwise unknown.

The second method 32] relies on a direct computation of the density at low frequency of the appropriate stress-tensor two-point function. As this computation is necessarily Euclidean, an analytic continuation is necessary. The values at a finite number of discrete Matsubara frequencies are not enough to analytically continue. An ansatz for the continuous density is also used here, which presents again a potentially large systematic uncertainty.

Calculations in IHQCD support a rise of the bulk viscosity near $T_{c}$, but the values are much smaller than previously expected. Studies of how this affects hydrodynamics at RHIC, [33] suggest that this implies a fall in radial and elliptic flow.

Another class of interesting experimental observables is associated with quarks, and comes under the label of "jet quenching". Central to this is the expectation that an energetic quark will loose energy very fast in the quark-gluon plasma because of strong coupling. This has as a side effect that back-to back jets are suppressed. Moreover if a pair of energetic quarks is generated near the plasma boundary then one will exit fast the plasma and register as an energetic jet, while the other will thermalize and its identity will disappear. This has been clearly observed at RHIC and used to study the energy loss of quarks in the quark-gluon plasma.

Heavy quarks are of extra importance, as their mass masks some low-energy strong interaction effects, and can be therefore cleaner probes of plasma energy loss. There are important electron observables at RHIC, 34] that can probe heavy-quark energy loss in the strongly coupled quark-gluon plasma. Such observables are also expected to play an important role in LHC [35].

A perturbative QCD approach to calculate the energy loss of a heavy quark in the plasma has been pursued by calculating radiative energy loss, [36]. However its application to the RHIC plasma has recently raised problems, based on comparison with data. A phenomenological coefficient used in such cases is known as the jet quenching coefficient $\hat{q}$, and is defined as the rate of change of the average value of transverse momentum square of a probe. Current fits, [34, 37], indicate that a value of order $10 \mathrm{GeV}^{2} / \mathrm{fm}$ or more is needed to describe the data while perturbative approaches are trustworthy at much lower values. 
Several attempts were made to compute quark energy loss in the holographic context, relevant for $\mathcal{N}=4 \mathrm{SYM}^{3}$. In some of them [39, 40] the jet-quenching coefficient $\hat{q}$ was calculated via its relationship to a light-like Wilson loop. Holography was then used to calculate the appropriate Wilson loop. The $\hat{q}$ obtained scales as $\sqrt{\lambda}$ and as the third power of the temperature,

$$
\hat{q}_{\text {conformal }}=\frac{\Gamma\left[\frac{3}{4}\right]}{\Gamma\left[\frac{5}{4}\right]} \sqrt{2 \lambda} \pi^{\frac{3}{2}} T^{3}
$$

A different approach chooses to compute the drag force acting a string whose UV endpoint (representing an infinitely heavy quark) is forced to move with constant velocity $v$, [9, 11, 12], in the context of $\mathcal{N}=4 \mathrm{SYM}$ plasma. The result for the drag force is

$$
F_{\text {conformal }}=\frac{\pi}{2} \sqrt{\lambda} T^{2} \frac{v}{\sqrt{1-v^{2}}}
$$

and is calculated by first studying the equilibrium configuration of the appropriate string world-sheet string and then calculating the momentum flowing down the string. This can be the starting point of a Langevin evolution system, as the process of energy loss has a stochastic character, as was first pointed out in [41] and more recently pursued in [42]- 48.

Such a system involves a classical force, that in this case is the drag force, and a stochastic noise that is taken to be Gaussian and which is characterized by a diffusion coefficient. There are two ingredients here that are novel. The first is that the Langevin evolution must be relativistic, as the quarks can be very energetic. Such relativistic systems have been described in the mathematical physics literature, 49] and have been used in phenomenological analyses of heavy-ion data, [37]. They are known however to have peculiar behavior, since demanding an equilibrium relativistic Boltzmann distribution, provides an Einstein relation that is pathological at large temperatures. Second, the transverse and longitudinal diffusion coefficients are not the same, [45]. A first derivation of such Langevin dynamics from holography was given in [45]. This has been extended in [48] where the thermal-like noise was associated and interpreted in terms of the world-sheet horizon that develops on the probe string.

Most of the transport properties mentioned above have been successfully computed in $\mathcal{N}=4 \mathrm{SYM}$ and a lot of debate is still waged as to how they can be applied to QCD in the appropriate temperature range, [11], [50], [51]. A holographic description of QCD has been elusive, and the best we have so far have been simple bottom up models.

In the simplest bottom-up holographic model known as AdS/QCD [7], the bulk viscosity is zero as conformal invariance is essentially not broken (the stress tensor is traceless), and the drag force and jet quenching essentially retain their conformal values.

In the soft-wall model [8], no reliable calculation can be done for glue correlators and therefore transport coefficients are ill-defined, as bulk equations of motion are not respected. Similar remarks hold for other phenomenologically interesting observables as the drag force and the jet quenching parameter.

The shear viscosity of IHQCD is the same as that of $\mathcal{N}=4$ SYM, as the model is a two derivative model. Although this is not a good approximation in the UV of QCD,

\footnotetext{
${ }^{3}$ Most are reviewed in [38].
} 
it is expected to be a good approximation in the energy range $T_{c} \leq T \leq 5 T_{c}$. The bulk viscosity in IHQCD rises near the phase transition but ultimately stays slightly below the shear viscosity. There is a general holographic argument that any (large-N) gauge theory that confines color at zero temperature should have an increase in the bulk viscosity-toentropy density ratio close to $T_{c}$.

The drag force on heavy quarks, and the associated diffusion times, can be calculated and found to be momentum depended as anticipated from asymptotic freedom. Numerical values of diffusion times are in the region dictated by phenomenological analysis of heavyion data. The medium-induced corrections to the quark mass (needed for the diffusion time calculation) can be calculated, and they result in a mildly decreasing effective quark mass as a function of temperature. This is consistent with lattice results. Finally, the jet-quenching parameter can be calculated and found to be comparable at $T_{c}$ to the one obtained by extrapolation from $\mathcal{N}=4 \mathrm{SYM}$. Its temperature dependence is however different and again reflects the effects of asymptotic freedom.

\section{The 5D model}

The holographic dual of large $N_{c}$ Yang Mills theory, proposed in [14], is based on a fivedimensional Einstein-dilaton model, with the action ${ }^{4}$ :

$$
S_{5}=-M_{p}^{3} N_{c}^{2} \int d^{5} x \sqrt{g}\left[R-\frac{4}{3}(\partial \Phi)^{2}+V(\Phi)\right]+2 M_{p}^{3} N_{c}^{2} \int_{\partial M} d^{4} x \sqrt{h} K .
$$

Here, $M_{p}$ is the five-dimensional Planck scale and $N_{c}$ is the number of colors. The last term is the Gibbons-Hawking term, with $K$ being the extrinsic curvature of the boundary. The effective five-dimensional Newton constant is $G_{5}=1 /\left(16 \pi M_{p}^{3} N_{c}^{2}\right)$, and it is small in the large- $N_{c}$ limit.

Of the $5 \mathrm{D}$ coordinates $\left\{x_{i}, r\right\}_{i=0 \ldots 3}, x_{i}$ are identified with the $4 \mathrm{D}$ space-time coordinates, whereas the radial coordinate $r$ roughly corresponds to the $4 \mathrm{D}$ RG scale. We identify $\lambda \equiv e^{\Phi}$ with the running 't Hooft coupling $\lambda_{t} \equiv N_{c} g_{Y M}^{2}$, up to an a priori unknown multiplicative factor ${ }^{5}, \lambda=\kappa \lambda_{t}$.

The dynamics is encoded in the dilaton potential ${ }^{6}, V(\lambda)$. The small- $\lambda$ and large$\lambda$ asymptotics of $V(\lambda)$ determine the solution in the UV and the IR of the geometry respectively. For a detailed but concise description of the UV and IR properties of the solutions the reader is referred to Section 2 of [19]. Here we will only mention the most relevant information:

\footnotetext{
${ }^{4}$ Similar models of Einstein-dilaton gravity were proposed independently in 16 to describe the finite temperature physics of large $N_{c}$ YM. They differ in the UV as the dilaton corresponds to a relevant operator instead of the marginal case we study here. The gauge coupling $e^{\Phi}$ also asymptotes to a constant instead of zero in such models.

${ }^{5}$ This relation is well motivated in the UV, although it may be modified at strong coupling (see Section 3). The quantities we will calculate do not depend on the explicit relation between $\lambda$ and $\lambda_{t}$.

${ }^{6}$ With a slight abuse of notation we will denote $V(\lambda)$ the function $V(\Phi)$ expressed as a function of $\lambda \equiv e^{\Phi}$.
} 
1. For small $\lambda, V(\lambda)$ is required to have a power-law expansion of the form:

$$
V(\lambda) \sim \frac{12}{\ell^{2}}\left(1+v_{0} \lambda+v_{1} \lambda^{2}+\ldots\right), \quad \lambda \rightarrow 0 .
$$

The value at $\lambda=0$ is constrained to be finite and positive, and sets the UV AdS scale $\ell$. The coefficients of the other terms in the expansion fix the $\beta$-function coefficients for the running coupling $\lambda(E)$. If we identify the energy scale with the metric scale factor in the Einstein frame, as in [14], we obtain:

$$
\begin{aligned}
& \beta(\lambda) \equiv \frac{d \lambda}{d \log E}=-b_{0} \lambda^{2}-b_{1} \lambda^{3}+\ldots \\
& b_{0}=\frac{9}{8} v_{0}, \quad b_{1}=\frac{9}{4} v_{1}-\frac{207}{256} v_{0}^{2} .
\end{aligned}
$$

2. For large $\lambda$, confinement and the absence of bad singularities ${ }^{7}$ require:

$$
V(\lambda) \sim \lambda^{2 Q}(\log \lambda)^{P} \quad \lambda \rightarrow \infty, \quad\left\{\begin{array}{l}
2 / 3<Q<2 \sqrt{2} / 3, \quad P \text { arbitrary } \\
Q=2 / 3, \quad P \geq 0
\end{array}\right.
$$

In particular, the values $Q=2 / 3, P=1 / 2$ reproduce an asymptotically-linear glueball spectrum, $m_{n}^{2} \sim n$, besides confinement. We will restrict ourselves to this case in what follows.

In the large $N_{c}$ limit, the canonical ensemble partition function of the model just described, can be approximated by a sum over saddle points, each given by a classical solution of the Einstein-dilaton field equations:

$$
\mathcal{Z}(\beta) \simeq e^{-\mathcal{S}_{1}(\beta)}+e^{-\mathcal{S}_{2}(\beta)}+\ldots
$$

where $\mathcal{S}_{i}$ are the euclidean actions evaluated on each classical solution with a fixed temperature $T=1 / \beta$, i.e. with euclidean time compactified on a circle of length $\beta$. There are two possible types of Euclidean solutions which preserve 3-dimensional rotational invariance. In conformal coordinates these are:

\section{Thermal gas solution,}

$$
d s^{2}=b_{o}^{2}(r)\left(d r^{2}+d t^{2}+d x_{m} d x^{m}\right), \quad \Phi=\Phi_{o}(r),
$$

with $r \in(0, \infty)$ for the values of $P$ and $Q$ we are using;

\section{Black-hole solutions,}

$$
d s^{2}=b(r)^{2}\left[\frac{d r^{2}}{f(r)}+f(r) d t^{2}+d x_{m} d x^{m}\right], \quad \Phi=\Phi(r),
$$

with $r \in\left(0, r_{h}\right)$, such that $f(0)=1$, and $f\left(r_{h}\right)=0$.

\footnotetext{
${ }^{7}$ We call "bad singularities" those that do not have a well defined spectral problem for the fluctuations without imposing extra boundary conditions.
} 
In both cases Euclidean time is periodic with period $\beta_{o}$ and $\beta$ respectively for the thermal gas and black-hole solution, and 3 -space is taken to be a torus with volume $V_{3 o}$ and $V_{3}$ respectively, so that the black-hole mass and entropy are finite ${ }^{8}$.

The black holes are dual to a deconfined phase, since the string tension vanishes at the horizon, and the Polyakov loop has non-vanishing expectation value ([52, 53]). On the other hand, the thermal gas background is confining.

The thermodynamics of the deconfined phase is dual to the 5D black-hole thermodynamics. The free energy, defined as

$$
\mathcal{F}=E-T S,
$$

is identified with the black-hole on-shell action; as usual, the energy $E$ and entropy $S$ are identified with the black-hole mass, and one fourth of the horizon area in Planck units, respectively.

The thermal gas and black-hole solutions with the same temperature differ at $O\left(r^{4}\right)$ :

$$
b(r)=b_{o}(r)\left[1+\mathcal{G} \frac{r^{4}}{\ell^{3}}+\ldots\right], \quad f(r)=1-\frac{C}{4} \frac{r^{4}}{\ell^{3}}+\ldots \quad r \rightarrow 0,
$$

where $\mathcal{G}$ and $C$ are constants with units of energy. As shown in [19] they are related to the enthalpy $T S$ and the gluon condensate $\left\langle\operatorname{Tr} F^{2}\right\rangle$ :

$$
C=\frac{T S}{M_{p}^{3} N_{c}^{2} V_{3}}, \quad \mathcal{G}=\frac{22}{3(4 \pi)^{2}} \frac{\left\langle\operatorname{Tr} F^{2}\right\rangle_{T}-\left\langle\operatorname{Tr} F^{2}\right\rangle_{o}}{240 M_{p}^{3} N_{c}^{2}} .
$$

Although they appear as coefficients in the UV expansion, $C$ and $\mathcal{G}$ are determined by regularity at the black-hole horizon. For $T$ and $S$ the relation is the usual one,

$$
T=-\frac{\dot{f}\left(r_{h}\right)}{4 \pi}, \quad S=\frac{\text { Area }}{4 G_{5}}=4 \pi\left(M_{p}^{3} N_{c}^{2} V_{3}\right) b^{3}\left(r_{h}\right) .
$$

For $\mathcal{G}$ the relation with the horizon quantities is more complicated and cannot be put in a simple analytic form. However, as discussed in [19], for each temperature there exist only specific values of $\mathcal{G}$ (each corresponding to a different black hole) such that the horizon is regular.

At any given temperature there can be one or more solutions: the thermal gas is always present, and there can be different black holes with the same temperature. The solution that dominates the partition function at a certain $T$ is the one with smallest free energy. The free energy difference between the black hole and thermal gas was calculated in 19. to be:

$$
\frac{\mathcal{F}}{M_{p}^{3} N_{c}^{2} V_{3}}=\frac{\mathcal{F}_{B H}-\mathcal{F}_{t h}}{M_{p}^{3} N_{c}^{2} V_{3}}=15 \mathcal{G}-\frac{C}{4} .
$$

For a dilaton potential corresponding to a confining theory, like the one we will assume, the phase structure is the following [19]:

\footnotetext{
${ }^{8}$ The periods and 3 -space volumes of the thermal gas solution are related to the black-hole solution values by requiring that the geometry of the two solutions are the same on the (regulated) boundary. See [19] for details.
} 
1. There exists a minimum temperature $T_{\min }$ below which the only solution is the thermal gas.

2. Two branches of black holes ("large" and "small") appear for $T \geq T_{\min }$, but the ensemble is still dominated by the confined phase up to a temperature $T_{c}>T_{\min }$

3. At $T=T_{c}$ there is a first order phase transition to the large black-hole phase. The system remains in the black-hole (deconfined) phase for all $T>T_{c}$.

In principle there could be more than two black-hole branches, but this will not happen with the specific potential we will use.

\section{Scheme dependence}

There are several sources of scheme dependence in any attempt to solve a QFT. Different parametrizations of the coupling constant (here $\lambda$ ) give different descriptions. However, physical statements must be invariant under such a change. In our case, reparametrizations of the coupling constant are equivalent to radial diffeomorphisms as we could use $\lambda$ as the radial coordinate.

In the holographic context, scheme dependence related to coupling redefinitions translates into field redefinitions for the bulk fields. As the bulk theory is on-shell, all on-shell observables (that are evaluated at the single boundary of space-time) are independent of the field redefinitions showing that scheme-independence is expected. Invariance under radial reparametrizations of scalar bulk invariants is equivalent to $\mathrm{RG}$ invariance. Because of renormalization effects, the boundary is typically shifted and in this case field redefinitions must be combined with appropriate radial diffeomorphisms that amount to RG-transformations.

Another source of scheme dependence in our setup comes from the choice of the energy function. Again we may also consider this as a radial coordinate and therefore it is subject to coordinate transformations. A relation between $\lambda$ and $E$ is the $\beta$-function,

$$
\frac{d \lambda}{d \log E}=\beta(\lambda)
$$

$\beta$ by definition transforms as a vector under $\lambda$ reparametrizations and as a form under $E$ reparametrizations. $\beta(\lambda)$ can therefore be thought of as a vector field implementing the change of coordinates from $\lambda$ to $E$ and vice-versa.

Physical quantities should be independent of scheme. They are quantities that are fully diffeomorphism invariant. If the gravitational theory had no boundary there would be no diffeomorphism invariant quantities, except for possible topological invariants. Since we have a boundary, diffeomorphism invariant quantities are defined at the boundary.

Note that scalar quantities are not invariant. To be invariant they must be scalar and constant. We therefore need to construct scalar functions that are invariant under changes of radial coordinates. 
We can fix this reparametrization invariance by picking a very special frame. For example choosing the (string) metric in the conformal frame

$$
d s^{2}=e^{2 A}\left[d r^{2}+d x^{\mu} d x_{\mu}\right] \quad, \quad \lambda(r)
$$

or in the domain-wall frame

$$
d s^{2}=d u^{2}+e^{2 A} d x^{\mu} d x_{\mu} \quad, \quad \lambda(u)
$$

fixes the radial reparametrizations almost completely. In conformal frame, common scalings of $r, x^{\mu}$ are allowed, corresponding to constant shifts of $A(r)$.

Eventually we are led to calculate and compare our results to other ways of calculating (like the lattice). Some outputs are easier to compare (for example correlators). Others are much harder as they are not invariant (like the value of the coupling at a given energy scale).

In the UV such questions are well understood. The asymptotic energy scale is fixed by comparison to conformal field theory examples. This is possible because the space is asymptotically $\mathrm{AdS}_{5}{ }^{9}$.

The coupling constant is also fixed to leading order from the coupling of the dilaton to $D_{3}$ branes (up to an overall multiplicative factor). Subleading (in perturbation theory) redefinitions of the coupling constant and the energy lead to changes in the $\beta$-function beyond two loops.

More in detail, as it has been described in [14, 15], the general form of the kinetic term for the gauge fields on a $D_{3}$ brane is expected to be:

$$
S_{F^{2}}=e^{-\Phi} Z(R, \xi) \operatorname{Tr}\left[F^{2}\right] \quad, \quad \xi \equiv-e^{2 \Phi} \frac{F_{5}^{2}}{5 !}
$$

where $Z(R, \xi)$ is an (unknown) function of curvature $R$ and the five-form field strength, $\xi$. At weak background fields, $Z \simeq-\frac{1}{4}+\cdots$. In the UV regime, expanding near the boundary in powers of the coupling $\lambda \equiv N_{c} e^{\Phi}$ we obtain, 15

$$
S_{F^{2}}=N_{c} \operatorname{Tr}\left[F^{2}\right] \frac{1}{\lambda}\left[Z\left(R_{*}, \xi_{*}\right)-\frac{Z_{\xi}\left(R_{*}, \xi_{*}\right)}{F_{\xi \xi}\left(R_{*}, \xi_{*}\right) \sqrt{\xi_{*}}} \frac{\lambda}{\ell}+\mathcal{O}\left(\lambda^{2}\right)\right]
$$

where $F(R, \xi)$ is the bulk effective action and $R_{*}, \xi_{*}$ are the boundary values for these parameters. Therefore the true 't Hooft coupling of QCD is

$$
\lambda_{\text {'t Hooft }}=-\frac{\lambda}{Z\left(R_{*}, \xi_{*}\right)}\left[1+\frac{Z_{\xi}\left(R_{*}, \xi_{*}\right)}{Z\left(R_{*}, \xi_{*}\right) F_{\xi \xi}\left(R_{*}, \xi_{*}\right) \sqrt{\xi_{*}}} \frac{\lambda}{\ell}+\mathcal{O}\left(\lambda^{2}\right)\right] .
$$

In the IR, more important changes can appear between our $\lambda$ and other definitions as for example in lattice calculations.

\footnotetext{
${ }^{9}$ As the dilaton is now not constant there is a non-trivial question: in which frame is the metric AdS. In 15] it was argued that this should be the case in the string frame. The difference of course between the string and Einstein frame is subleading in the UV as the coupling constant vanishes logarithmically. But this may not be the case in the IR where we have very few criteria to check. In the model we are using we impose that the space is asymptotically AdS in the Einstein frame as this is the only choice consistent with the whole framework.
} 
In the region of strong coupling we know much less in order to be guided concerning the correct definition of the energy. We can obtain some hints however by comparing with lattice results. ${ }^{10}$ In particular, based on lattice calculations using the Schröndiger functional approach [54], it is argued that at long distance $L$ the 't Hooft coupling constant scales as

$$
\lambda_{\text {lat }} \sim e^{m L} \quad, \quad m \simeq \frac{3}{4} m_{0^{++}} .
$$

This was based on a specific definition of the coupling constant, and length scale on the lattice as well as on numerical data, and some general expectations on the fall-off of correlations in a massive theory. This suggests an IR $\beta$ function of the form

$$
L \frac{d \lambda}{d L}=\lambda \log \frac{\lambda}{\lambda_{0}} \quad, \quad \lambda=\lambda_{0} e^{m L} .
$$

On the other hand our $\beta$-function at strong coupling uses the UV definition of energy, $\log E=A_{E}$ (the scale factor in the Einstein frame), $E \sim 1 / L$ and is

$$
L \frac{d \lambda}{d L}=\frac{3}{2} \lambda\left[1+\frac{3}{4} \frac{a-1}{a} \frac{1}{\log \lambda}+\cdots\right] \quad, \quad \lambda \simeq\left(\frac{L}{L_{0}}\right)^{\frac{3}{2}} .
$$

where $a$ is a parameter in the IR asymptotics of the potential. The case we consider as best fitting YM is $a=2$ as then the asymptotic glueball trajectories are linear..

Consider now taking as length scale the string scale factor $e^{A_{s}}$ in the IR. ${ }^{11}$ Since it increases, it is consistent to consider it as a monotonic function of length. From its relation to the Einstein scale factor $A_{s}=A_{E}+\frac{2}{3} \log \lambda$ and (3.9) we obtain

$$
\frac{d \lambda}{d A_{s}}=\frac{2 a}{a-1} \lambda \log \lambda+\cdots
$$

Therefore if we define as length scale in the IR

$$
\log L=\frac{2 a}{a-1} A_{s} \quad \rightarrow \quad L=\left(e^{A_{s}}\right)^{\frac{2 a}{a-1}}
$$

we obtain a running of the coupling compatible with the given lattice scheme. Note however that $L=\left(e^{A_{s}}\right)^{\frac{2 a}{a-1}}$ cannot be a global choice but should be only valid in the IR. The reason is that this function is not globally monotonic.

We conclude this section by restating that physical observables are independent of scheme. But observables like the 't Hooft coupling constant do depend on schemes, and it is obvious that our scheme is very different from lattice schemes in the IR.

\footnotetext{
${ }^{10}$ We would like to thank K. Kajantie for asking the question, suggesting to compare with lattice data, and providing the appropriate references.

${ }^{11}$ The string scale factor is not a monotonic function on the whole manifold, 14] and this is the reason that it was not taken as a global energy scale. In particular in the UV, $e^{A_{s}}$ decreases until it reaches a minimum. The existence of the minimum is crucial for confinement. After this minimum $e^{A_{s}}$ increases and diverges at the IR singularity.
} 


\section{The potential and the parameters of the model}

We will make the following ansatz for the potential ${ }^{12}$,

$$
V(\lambda)=\frac{12}{\ell^{2}}\left\{1+V_{0} \lambda+V_{1} \lambda^{4 / 3}\left[\log \left(1+V_{2} \lambda^{4 / 3}+V_{3} \lambda^{2}\right)\right]^{1 / 2}\right\},
$$

which interpolates between the two asymptotic behaviors (2.2) for small $\lambda$ and (2.4) for large $\lambda$, with $Q=2 / 3$ and $P=1 / 2$. Not all the parameters entering this potential have physical relevance. Below we will discuss the independent parameters of the model, and their physical meaning.

The normalization of the coupling constant $\lambda$. As discussed in the previous section, the relation between the bulk field $\lambda(r)$ and the physical QCD 't Hooft coupling $\lambda_{t}=$ $g_{Y M}^{2} N_{c}$ is a priori unknown. In the UV, the identification of the $D 3$-brane coupling to the dilaton implies that the relation is linear, and depends on an a priori unknown coefficient $\kappa$, defined as:

$$
\lambda=\kappa \lambda_{t} .
$$

The coefficient $\kappa$ can in principle be identified by relating the perturbative UV expansion of the Yang-Mills $\beta$-function, to the holographic $\beta$-function for the bulk field $\lambda$ :

$$
\begin{array}{ll}
\beta\left(\lambda_{t}\right)=-\beta_{0} \lambda_{t}^{2}-\beta_{1} \lambda_{t}^{3}+\ldots & \beta_{0}=\frac{22}{3(4 \pi)^{2}}, \beta_{1}=\frac{51}{121} \beta_{0}^{2}, \ldots \\
\beta(\lambda)=-b_{0} \lambda^{2}-b_{1} \lambda^{3}+\ldots, & b_{0}=\frac{9}{8} v_{0}, b_{1}=\frac{9}{4} v_{1}-\frac{207}{256} v_{0}^{2} \ldots .
\end{array}
$$

The two expressions (4.3) and (4.4) are consistent with a linear relation as in (4.2), and expanding the identity $\kappa \beta_{t}\left(\lambda_{t}\right)=\beta\left(\kappa \lambda_{t}\right)$ to lowest order leads to:

$$
\kappa=\beta_{0} / b_{0}
$$

Therefore, to relate the bulk field $\lambda$ to the true coupling $\lambda_{t}$ one looks at the linear term in the expansion of the potential. More generally, the other $\beta$ function coefficients are related by $\beta_{n}=\kappa^{n+1} b_{n}$, and the combinations $b_{n} / b_{0}^{n+1}=\beta_{n} / \beta_{0}^{n+1}$ are $\kappa$-independent (however they are scheme-dependent for $n \geq 2$ ).

As discussed in Section 3, the introduction of the coefficient $\kappa$ amounts to a field redefinition and therefore its precise value does not affect physical (scheme-independent) quantities. In this sense, $\kappa$ is not a parameter that can be fixed by matching some observable computed in the theory. Assuming the validity of the relation (4.2), we could eventually fix $\kappa$ by matching a RG-invariant (but scheme-dependent) quantity, e.g. $\lambda$ at a given energy scale.

However, as we discuss later in this section, rescaling $\lambda$ in the potential (thus changing $\kappa)$ affects other parameters in the models, that are defined in the string frame, e.g. the fundamental string length $\ell_{s}$ : if we hold the physical QCD string tension fixed, the ratio $\left(\ell_{s} / \ell\right)$ scales with degree $-2 / 3$ under a rescaling of $\kappa$.

\footnotetext{
${ }^{12}$ Further studies of IHQCD with different potentials can be found in 55.
} 
An important point to keep in mind, is that the simple linear relation (4.2) may be modified at strong coupling, but again this does not have any effect on physical observables. As long as we compute $R G$-invariant and scheme-independent quantities, knowledge of the exact relationship $\lambda=F\left(\lambda_{t}\right)$ is unnecessary.

The AdS scale $\ell$. This is set by the overall normalization of the potential, and its choice is equivalent to fixing the unit of energy. It does not enter dimensionless physical quantities. As usual the AdS length at large $N_{c}$ is much larger than the Planck length $\left(\ell_{p} \sim 1 /\left(M_{p} N_{c}^{2 / 3}\right)\right.$, independently of the 't Hooft coupling.

The UV expansion coefficients of $V(\lambda)$. They can be fixed order by order by matching the Yang-Mills $\beta$-function. We impose this matching up to two-loops in the perturbative expansion, i.e. $O\left(\lambda^{3}\right)$ in $\beta(\lambda)$. One could go to higher orders by adding additional powers of $\lambda$ inside the logarithm, but since our purpose is not to give an accurate description of the theory in the UV, we choose not to introduce extra parameters ${ }^{13}$.

Identifying the energy scale with the Einstein frame scale factor, $\log E \equiv \log b(r)$, we have the relation (4.4) between the $\beta$-function coefficients and the expansion parameters of $V(\lambda)$, with

$$
v_{0}=V_{0}, \quad v_{1}=V_{1} \sqrt{V_{2}} .
$$

The term proportional to $V_{2}$ in eq. (4.1) is needed to reproduce the correct value of the quantity $b_{1} / b_{0}^{2}=\beta_{1} / \beta_{0}^{2}=51 / 121$, which is invariant under rescaling of $\lambda$. Thus, $V_{2}$ is not a free parameter, but is fixed in terms of $V_{0}$ and $V_{1}$ by:

$$
V_{2}=b_{0}^{4}\left(\frac{23+36 b_{1} / b_{0}^{2}}{81 V_{1}}\right)^{2}, \quad b_{0}=\frac{9}{8} V_{0}, \quad \frac{b_{1}}{b_{0}^{2}}=\frac{51}{121} .
$$

As explained earlier in this section, when discussing the normalization of the coupling, fixing the coefficient $V_{0}$ is the same as fixing the normalization $\kappa$ through eq. (4.5). As we argued, the actual value of $\kappa$ should not have any physical consequences, so it is tempting to set $V_{0}=1$ by a field redefinition, $\lambda \rightarrow \lambda / V_{0}$ and eliminate this parameter altogether.

In fact, most of the quantities we will compute are not sensitive to the value of $V_{0}$, but for certain quantities, such as the string tension, some extra care is needed. In general, we can ask whether two models of the same form (2.1), but with different potentials $V(\lambda)$ and $\tilde{V}(\lambda)$, such that $\tilde{V}(\lambda)=V(\alpha \lambda)$ for some constant $\alpha$, lead to different physical predictions. As we can change from one model to the other simply by a field redefinition $\lambda \rightarrow \alpha \lambda$ ( this has no effect on the other terms in the action in the Einstein frame, eq. (2.1) ), clearly the two potentials lead to the same result for any physical quantity that can be computed unambiguously from the Einstein frame action, e.g. dimensionless ratios between glueball masses, critical temperature, latent heat etc.

However a rescaling of $\lambda$ does affect the string frame metric, since the latter explicitly contains factors of $\lambda: b_{s}(r)=b(r) \lambda^{2 / 3}$ 14 thus, under the rescaling $\lambda \rightarrow \alpha \lambda, b_{s}(r) \rightarrow$ $\alpha^{2 / 3} b_{s}(r)$. This means that any dimensionless ratio of two quantities, such that one of them remains fixed in the string frame and the other in the Einstein frame, will depend on

\footnotetext{
${ }^{13}$ Moreover, higher order $\beta$-function coefficients are known to be scheme-dependent.
} 
$\alpha$. An example of this is the ratio $\ell_{s} / \ell$, where $\ell_{s}$ is the string length, that we will discuss shortly.

Therefore, we can safely perform a field redefinition and set $V_{0}$ to a given value, as long as we are careful when computing quantities that depend explicitly on the fundamental string length.

Bearing this caveat in mind, we will choose a normalization such that $b_{0}=\beta_{0}$, i.e.

$$
V_{0}=\frac{8}{9} \beta_{0}
$$

so that the normalization of $\lambda$ in the UV matches the physical Yang-Mills coupling. With this choice, out of the four free parameters $V_{i}$ appearing in 4.1) only $V_{1}$ and $V_{3}$ play a non-trivial role ( $V_{2}$ being fixed by eq. (4.7)).

The 5D Planck scale $M_{p} . \quad M_{p}$ appears in the overall normalization of the $5 \mathrm{D}$ action (2.1). Therefore it enters the overall scale of quantities derived by evaluating the on-shell action, e.g. the free energy and the black-hole mass. It also sets the conversion factor between the entropy and horizon area. $M_{p}$ cannot be fixed directly as we lack a detailed underlining string theory for YM. To obtain quantitative predictions, $M_{p}$ must be fixed in terms of the other dimension-full quantity of the model, namely the AdS scale $\ell$. As shown in [19] this can be done by imposing that the high-temperature limit of the black-hole free energy be that of a free gluon gas with the correct number of degrees of freedom ${ }^{14}$. This requires:

$$
\left(M_{p} \ell\right)^{3}=\frac{1}{45 \pi^{2}} .
$$

The string length. In the non-critical approach the relation between the string length $\ell_{s}$ and the 5D Planck length (or the AdS length $\ell$ ) is not known from first principles. The string length does not appear explicitly in the 2-derivative action (2.1), but it enters quantities like the static quark-antiquark potential. The ratio $\ell_{s} / \ell$ can be fixed phenomenologically to match the lattice results for the confining string tension.

More in detail, the relation between the fundamental and the confining string tensions $T_{f}$ and $\sigma$ is given by:

$$
\sigma=T_{f} b^{2}\left(r_{*}\right) \lambda^{4 / 3}\left(r_{*}\right),
$$

where $r_{*}$ is the point where the string frame scale factor, $b_{s}(r) \equiv b(r) \lambda^{2 / 3}(r)$, has its minimum. Fixing the confining string tension by comparison with the lattice result we can find $T_{f}$ (more precisely, the dimensionless quantity $T_{f} \ell^{2}$, since the overall scale of the metric depends on $\ell$ ). The string length is in turn given by $\ell_{s} / \ell=1 / \sqrt{2 \pi T_{f} \ell^{2}}$.

As is clear from eq. (4.10), rescaling $\lambda \rightarrow \alpha \lambda$, keeping the value of the QCD string tension $\sigma$ and of the AdS scale $\ell$ fixed, affects the fundamental string length in AdS units as $\ell_{s} / \ell \rightarrow \alpha^{-2 / 3}\left(\ell_{s} / \ell\right)$. Therefore two models $a$ and $b$, defined in the Einstein frame by

\footnotetext{
${ }^{14}$ Note that this is conceptually different from the $\mathcal{N}=4$ case. There, near the boundary, the theory is strongly coupled and this number must be calculated in string theory. It is different by a factor of $3 / 4$ from the free sYM answer. Here near the boundary the theory is free. Therefore the number of degrees of freedom can be directly inferred.
} 
eq. (2.1), but with potentials related by $V_{b}(\lambda)=V_{a}(\alpha \lambda)$, must have different fundamental string tensions in order to reproduce the same result for the QCD string tension. The quantity $\ell_{s} / \ell$ therefore depends on the value of $V_{0}$.

Integration constants. Besides the parameters appearing directly in the gravitational action, there are also other physically relevant quantities that label different solutions to the 5 -th order system of field equations. Any solution is characterized by a scale $\Lambda$, the temperature $T$ and a value for the gluon condensate $\mathcal{G}$, that correspond to three of the five independent integration constants. ${ }^{15}$

Regularity at the horizon fixes $\mathcal{G}$ as a function of $T$, so that effectively the gluon condensate is a temperature-dependent quantity.

The quantity $\Lambda$ controls the asymptotic form of the solution, as it enters the dilaton running in the $\mathrm{UV}: \lambda \simeq-\left(b_{0} \log r \Lambda\right)^{-1}$. It can be defined in a reparametrization invariant way as:

$$
\Lambda=\ell^{-1} \lim _{\lambda \rightarrow 0}\left\{b(\lambda) \frac{\exp \left[-\frac{1}{b_{0} \lambda}\right]}{\lambda^{b_{1} / b_{0}^{2}}}\right\},
$$

and it is fixed once we specify the value of the scale factor $b(\lambda)$ at a given $\lambda_{0}$.

Every choice of $\Lambda$ corresponds to an inequivalent class of solutions, that differ by UV boundary conditions. Each class is thermodynamically isolated, since solutions with different $\Lambda$ 's have infinite action difference. Thus, in the canonical partition sum we need to consider only solutions with a fixed value of $\Lambda$. However, this choice is merely a choice of scale, as solutions with different $\Lambda$ 's will give the same predictions for any dimensionless quantity. In short, $\Lambda$ is the holographic dual to the QCD strong coupling scale: it is defined by the initial condition to the holographic RG equations, and does not affect dimensionless quantities such as mass ratios, etc. Therefore, as long as all solutions we consider obey the same UV asymptotics, the actual value of $\Lambda$ is immaterial, since the physical units of the system can always be set by fixing $\ell$.

To summarize, the only nontrivial phenomenological parameters we have at our disposal are $V_{1}$ and $V_{3}$ appearing in (4.1). The other quantities that enter our model are either fixed by the arguments presented in this section, or they only affect trivially (e.g. by overall rescaling that can be absorbed in the definition of the fundamental string scale) the physical quantities.

In the next section we present a numerical analysis of the solutions and thermodynamics of the model defined by eq. (4.1), and show that for an appropriate choice of the parameters it reproduces the lattice results for the Yang-Mills deconfinement transition and high-temperature phase as well as the zero temperature glueball data.

\footnotetext{
${ }^{15}$ The remaining two are the value $f(0)$ which should be set to one for the solution (2.7) to obey the right UV asymptotics, and an unphysical degree of freedom in the reparametrization of the radial coordinate.
} 


\section{Matching the thermodynamics of large- $N_{c}$ YM}

Assuming a potential of the form (4.1), we look for values of the parameters such that the thermodynamics of the $5 \mathrm{D}$ model match the lattice results for the thermodynamics of $4 \mathrm{D}$ YM. As explained in Section 3, we set $V_{0}$ and $V_{2}$ as in eqs. (4.8) and (4.7), respectively, with $b_{0}=\beta_{0}=22 / 3(4 \pi)^{-2}$.

We then vary $V_{1}$ and $V_{3}$ only. We fix these parameters by looking at thermodynamic quantities corresponding to the latent heat per unit volume, and the pressure at one value of the temperature above the transition, which we take as $2 T_{c}$.

It is worth remarking that $V_{1}$ and $V_{3}$ are phenomenological parameters that we use to fit dimensionless QCD quantities. The single (dimension-full) parameter of pure YangMills, the strong coupling scale, is an extra input that fixes the overall energy scale of our solution.

Using the numerical method explained in [22], for each set of parameters $\left(V_{1}, V_{3}\right)$ we numerically generate black-hole solutions for a range of values of $\lambda_{h}$, then from the metric at the horizon and its derivative we extract the temperature and entropy functions $T\left(\lambda_{h}\right)$ and $S\left(\lambda_{h}\right)$, and the function $\mathcal{F}\left(\lambda_{h}\right)$ from the integrated form of the first law,

$$
\mathcal{F}\left(\lambda_{h}\right)=\int_{\lambda_{h}}^{+\infty} \mathrm{d} \bar{\lambda}_{h} S\left(\bar{\lambda}_{h}\right) \frac{\mathrm{d} T\left(\bar{\lambda}_{h}\right)}{\mathrm{d} \bar{\lambda}_{h}}
$$

Here $S\left(\lambda_{h}\right)$ is given by (2.11) and both the large black hole and small black-hole branches are needed in order the get the full result for the free energy. This is because the integral in (5.1) extends to $+\infty$, entering deeply in the small black-hole branch.

The behavior of the thermodynamic functions is shown in Figures 2 , 3 and 4 , for the best fit parameter values that we discuss below. One can see the existence of a minimal temperature $T_{\min }=T\left(\lambda_{\text {min }}\right)$, and a critical value $\lambda_{c}$ where $\mathcal{F}$ changes sign. The resulting function $\mathcal{F}(T)$ is shown in Figure 1 .

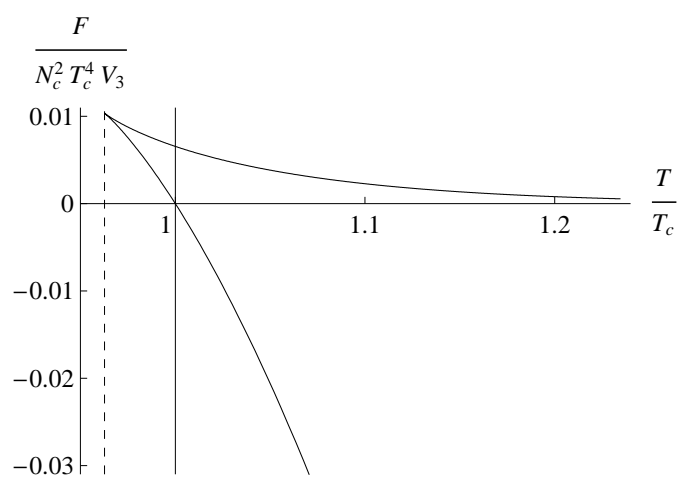

Figure 1: The Free energy density (in units of $T_{c}$ ) as a function of $T / T_{c}$, for $V_{1}=14$ and $V_{3}=170$. The vertical lines correspond to the critical temperature (solid) and the minimum black-hole temperature (dashed).

The phase transition is first order, and the latent heat per unit volume $L_{h}$, normalized by $N_{c}^{2} T_{c}^{4}$, is given by the derivative of the curve in Fig. 11 at $T / T_{c}=1$. Equivalently, $L_{h}$ 
is proportional to the jump in the entropy density $s=S / V_{3}$ at the phase transition from the thermal gas (whose entropy is of $O(1)$, in the limit $N_{c} \rightarrow \infty$ ) to the black hole (whose entropy scales as $N_{c}^{2}$ in the same limit): thus, in the large $N_{c}$ limit,

$$
L_{h} \equiv T \Delta s \simeq T_{c} s\left(\lambda_{c}\right)
$$

up to terms of $O\left(1 / N_{c}^{2}\right)$.

To fix $V_{1}$ and $V_{3}$ we compare our results to the data of G. Boyd et al. [56]. The relevant quantities to compare are the dimensionless ratios $p(T) / T^{4}, e(T) / T^{4}$ and $s(T) / T^{3}$, where $p=\mathcal{F} / V_{3}$ is the pressure, and $e=p+T s$ is the energy density. Lattice results for these functions are available in the range $T=T_{c} \sim 5 T_{c}$, and can be seen in Figure 7 of [56]. The analysis of [56] correspond to $N_{c}=3$, but one expects that the thermodynamic functions do not change to much for large $N_{c}{ }^{16}$.

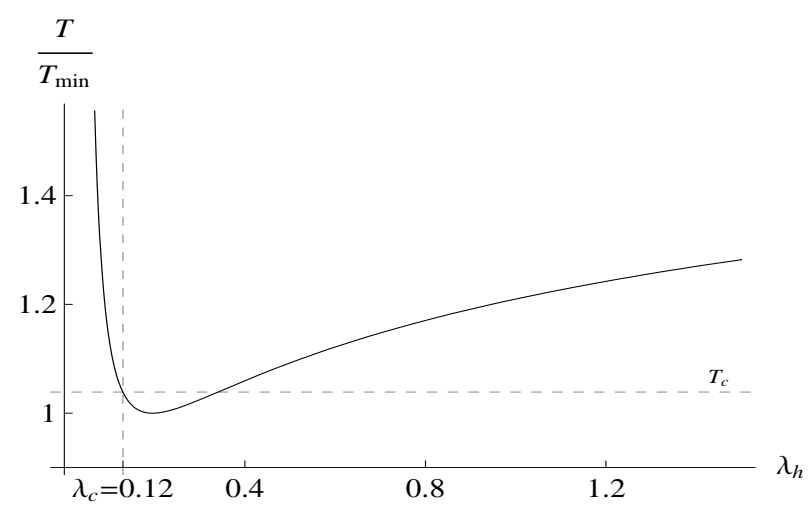

Figure 2: Temperature in units of $T_{\min }$, as a function of $\lambda_{h}$, for $V_{1}=14$ and $V_{3}=170$. The dashed horizontal and vertical lines indicate the critical temperature and the critical value of the dilaton field at at the horizon

An additional quantity of relevance is the value for the "dimensionless" latent heat per unit volume, $L_{h} / T_{c}^{4}$ which for large $N_{c}$ was found in [59] to be $\left(L_{h} / T_{c}^{4}\right)_{l a t}=0.31 N_{c}^{2}$. The result for $N_{c}^{2}=3$ is slightly lower $\left(\simeq 0.28 N_{c}^{2}\right)$.

As already noted in 18, 19, the qualitative features of the thermodynamic functions are generically reproduced in our setup: the curves $3 p(T) / T^{4}, e(T) / T^{4}$ and $3 s(T) / 4 T^{3}$ increase starting at $T_{c}$, then (very slowly) approach the constant free field value $\pi^{2} N_{c}^{2} / 15$ (given by the Stefan-Boltzmann law) as $T$ increases. By computing the thermodynamic functions for various sets of values of $V_{1}$ and $V_{3}$ we obtain that:

1) $V_{1}$ roughly controls the height reached by the curves $p(T) / T^{4}, e(T) / T^{4}$ and $s(T) / T^{3}$ at large $T / T_{c}(\sim$ a few $)$ : for larger $V_{1}$ the curves approach the free field limit faster;

2) $V_{3}$ does not affect much the height of the curves at large $T / T_{c}$, but on the other hand it changes the latent heat, which is increasing as $V_{3}$ decreases.

The best fit corresponds to the values

\footnotetext{
${ }^{16}$ See e.g. [57], in which results for $N_{c}=8$ do not different significantly from those for $N_{c}=3$ as well as the recent high-precision data by Panero, [58].
} 


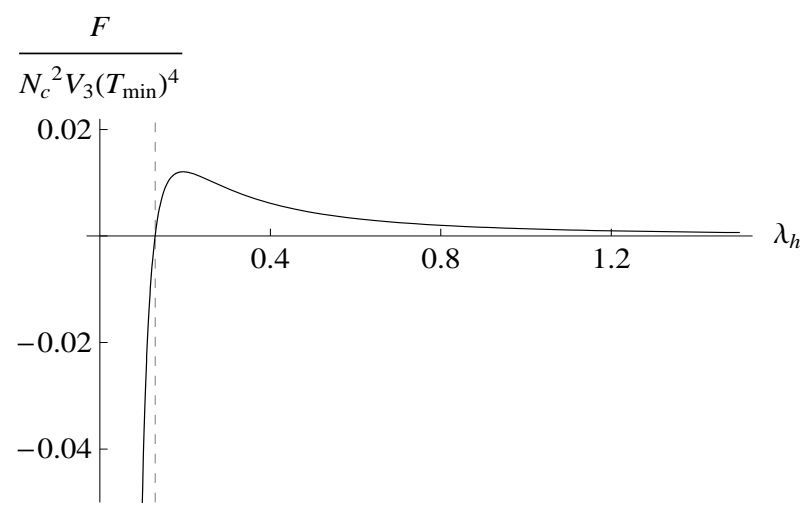

Figure 3: The free energy density in units of $T_{m i n}$, as a function of $\lambda_{h}$

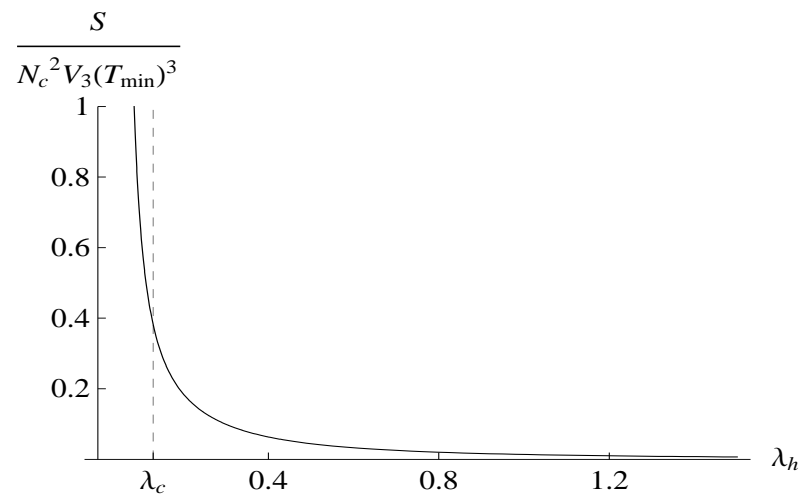

Figure 4: Entropy density in units of $T_{\min }$, as a function of $\lambda_{h}$

$$
V_{1}=14 \quad V_{3}=170 .
$$

Below we discuss the values of various physical quantities ( both related to thermodynamics, and to zero-temperature properties) obtained with this choice of parameters.

\subsection{Latent heat and equation of state}

The comparison between the curves $p(T) / T^{4}, e(T) / T^{4}$ and $s(T) / T^{3}$ obtained in our models with (5.3), and the lattice results [56] is shown in Figure 5. The match is remarkably good for $T_{c}<T<2 T_{c}$, and deviates slightly from the lattice data in the range up to $5 T_{c}$.

The latent heat we obtain is:

$$
L_{h} / T_{c}^{4}=0.31 N_{c}^{2},
$$

which matches the lattice result for $N_{c} \rightarrow \infty$ [59.

An interesting quantity is the trace anomaly $(e-3 p) / T^{4}$, (also known as interaction measure), that indicates the deviation from conformality, and it is proportional to the thermal gluon condensate. The trace anomaly in our setup is shown, together with the 


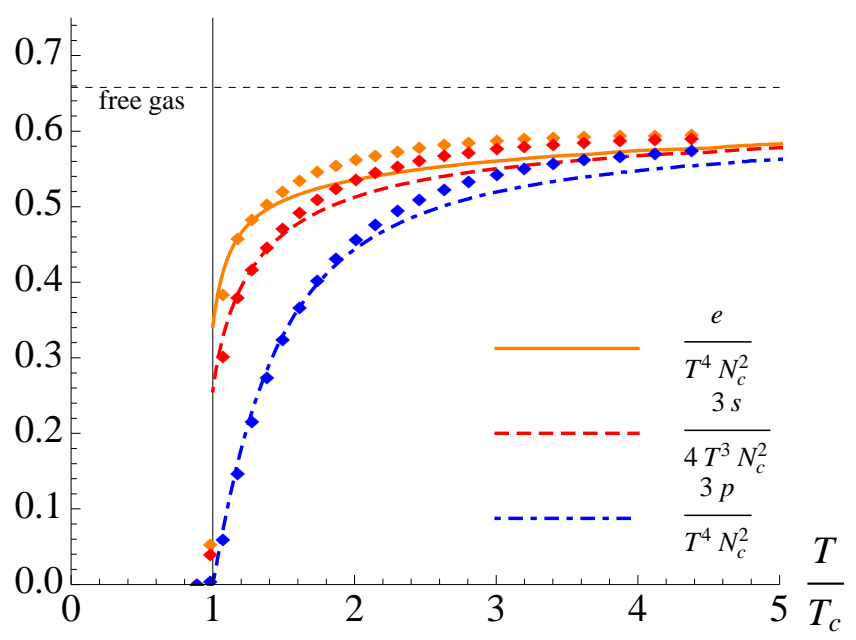

Figure 5: Temperature dependence of the dimensionless thermodynamic densities $s / T^{3}$ (light blue), $p / T^{4}$ (dark blue) and $e / T^{4}$ (green), normalized such that they reach the common limiting value $\pi^{2} / 15$ (dashed horizontal line) as $T \rightarrow \infty$. The dots correspond to the lattice data for $N_{c}=3$ [56.

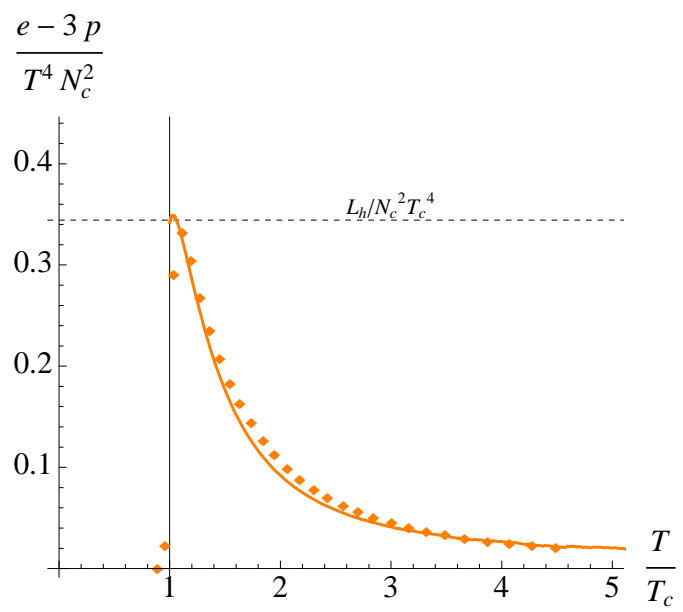

Figure 6: The trace anomaly as a function of temperature in the deconfined phase of the holographic model (solid line) and the corresponding lattice data [56 for $N_{c}=3$ (dots). The peak in the lattice data slightly above $T_{c}$ is expected to be an artifact of the finite lattice volume. In the infinite volume limit the maximum value of the curve is at $T_{c}$, and it equals $L_{h} / N_{c}^{2} T_{c}^{4}$.

corresponding lattice data, in figure (6), and the agreement is again very good. Our results agree even better with recent high-precision lattice calculations of the thermodynamics functions done by Panero at different values of $N_{c}$ up to $N_{c}=8$, [58]. In figure 0 a comparison (taken from [58]) of the normalized interaction measure with lattice results for different $N_{c}$ is shown.

We also compute the specific heat per unit volume $c_{v}$, and the speed of sound $c_{s}$ in 
Trace of the energy-momentum tensor

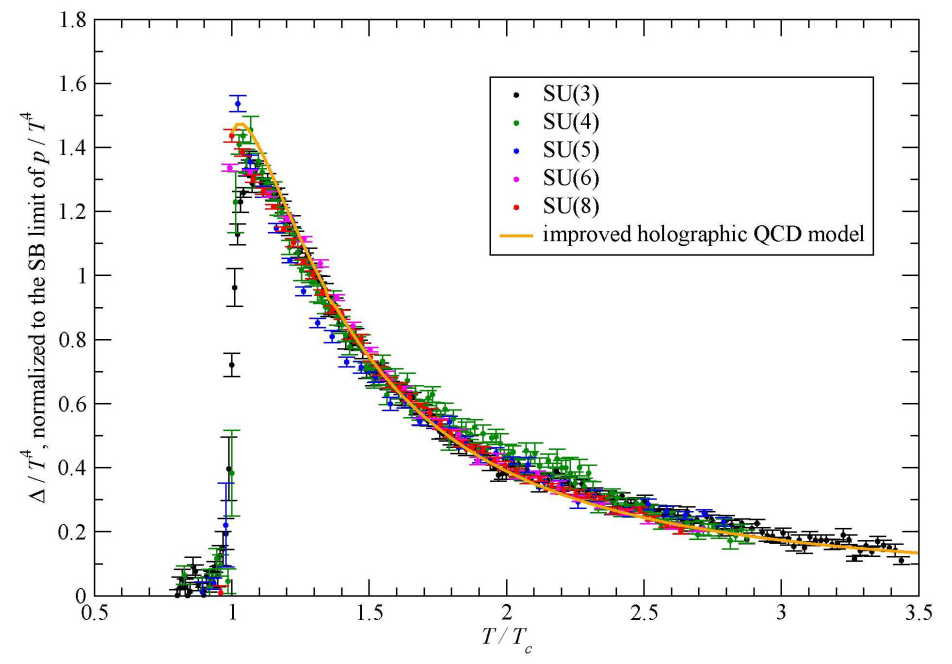

Figure 7: The rescaled trace anomaly (so that it is $N_{c}$-independent) as a function of temperature in the deconfined phase of the holographic model (solid line) and the corresponding recent high precision lattice data taken from 58 for different $N_{c}$. The errors shown are statistical only.

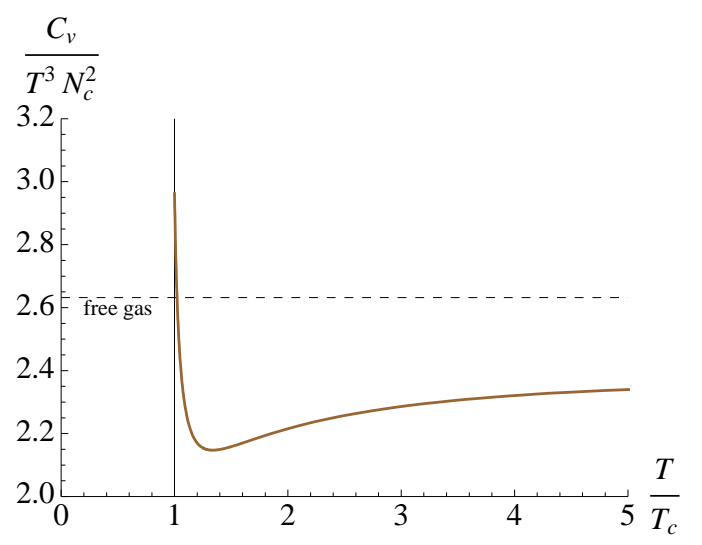

Figure 8: The specific heat (divided by $T^{3}$ ), as a function of temperature, in the deconfined phase of the holographic model.

the deconfined phase, by the relations

$$
c_{v}=-T \frac{\partial^{2} F}{\partial T^{2}}, \quad c_{s}^{2}=\frac{s}{c_{v}} .
$$

These are shown in Figures 8 and 9 respectively. The speed of sound is shown together with the lattice data, and the agreement is remarkable.

\subsection{Glueball spectrum}

In [14], the single phenomenological parameters of the potential was fixed by looking at the 


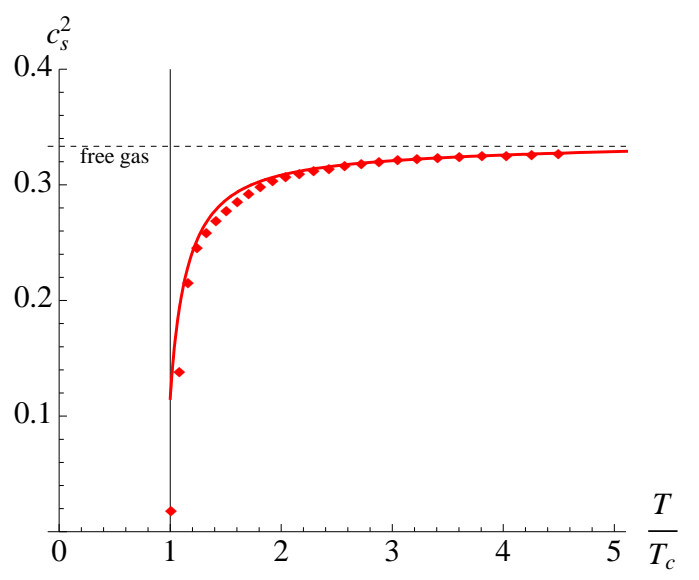

Figure 9: The speed of sound in the deconfined phase, as a function of temperature, for the holographic model (solid line) and the corresponding lattice data [56 for $N_{c}=3$ (dots). The dashed horizontal line indicates the conformal limit $c_{s}^{2}=1 / 3$.

zero-temperature spectrum, i.e. by computing various glueball mass ratios and comparing them to the corresponding lattice results. The masses are computed by deriving the effective action for the quadratic fluctuations around the background, [60] and subsequently reducing the dynamics to four dimensions.

The associated thermodynamics for this potential was studied in [18] which was in qualitative agreement with lattice QCD results, but not in full quantitative agreement. This is due to the fact that the thermodynamics depends more on the details of the potential than the glueball spectrum for the main Regge trajectories. Here we use the potential (4.1), but with the two phenomenological parameters $V_{1}$ and $V_{3}$ already determined by the thermodynamics (5.3).

The glueball spectrum is obtained holographically as the spectrum of normalizable fluctuations around the zero-temperature background. As explained in the introduction, and motivated in [14, 15], here we consider explicitly the 5D metric, one scalar field (the dilaton), and one pseudoscalar field (the axion). As a consequence, the only normalizable fluctuations above the vacuum correspond to spin 0 and spin 2 glueballs ${ }^{17}$ (more precisely, states with $J^{P C}=0^{++}, 0^{-+}, 2^{++}$), each species containing an infinite discrete tower of excited states.

In 4D YM there are many more operators generating glueballs, corresponding to different values of $J^{P C}$, that are not considered here. These are expected to correspond holographically to other fields in the noncritical string spectrum (e.g. form fields, which may yield spin 1 and CP-odd spin 2 states) and to higher string states that provide higherspin glueballs. As the main focus is in reproducing the YM thermodynamics in detail rather than the entire glueball spectrum, we choose not to include these states $^{18}$. Therefore we only compare the mass spectrum obtained in our model to the lattice results for

\footnotetext{
${ }^{17}$ Spin 1 excitations of the metric can be shown to be non-normalizable.

${ }^{18} \mathrm{~A}$ further reason is that, unlike the scalar and (to some extent) the pseudoscalar sector that we are considering, the action governing the higher Regge slopes is less and less universal as one goes to higher
} 
the lowest $0^{++}, 0^{-+}, 2^{++}$glueballs and their available excited states. These are limited to one for each spin 0 species, and none for the spin 2, in the study of [61], which is the one we use for our comparison. This provides two mass ratios in the CP-even sector and two in the CP-odd sector.

The glueball masses are computed by first solving numerically the zero-temperature Einstein's equations, by setting $f(r)=1$, and using the resulting metric and dilaton to setup an analogous Schrödinger problem for the fluctuations, [14]. The results for the parity-conserving sector are shown in Table 1, and are in good agreement with those reported by [61] for $N_{c}=3$, whereas the results reported by [62] for large $N_{c}$ are somewhat larger. The CP-violating sector (axial glueballs) will be discussed separately.

Table 1: Glueball Masses

\begin{tabular}{|c|c|c|c|}
\hline & HQCD & $N_{c}=3[61]$ & $N_{c}=\infty[62]$ \\
\hline \hline$m_{0^{*++}} / m_{0^{++}}$ & 1.61 & $1.56(11)$ & $1.90(17)$ \\
\hline$m_{2^{++}} / m_{0^{++}}$ & 1.36 & $1.40(4)$ & $1.46(11)$ \\
\hline \hline
\end{tabular}

We should add that there are other lattice studies (see e.g. [63]) that report additional excited states. Our mass ratios offer a somewhat worse fit of the mass ratios found in 63 (whose results are not entirely compatible with those of [61] for the states the two studies have in common). We should stress however that reproducing the detailed glueball spectrum is secondary here since the main focus is thermodynamics. However, the comparison of our spectrum to the existing lattice results shows that our model provides a good global fit to $4 \mathrm{D} \mathrm{YM}$ also with respect to quantities beyond thermodynamics.

Unlike the various mass ratios, the value of any given mass in AdS-length units (e.g. $m_{0++} \ell$ ) does depend on the choice of integration constants in the UV, i.e. on the value of $b_{U V}$ and $\lambda_{U V}$. Therefore its numerical value does not have an intrinsic meaning. However it can be used as a benchmark against which all other dimension-full quantities can be measured (provided one always uses the same UV b.c. ). On the other hand, given a fixed set of initial conditions, asking that $m_{0++}$ matches the physical value (in $\mathrm{MeV}$ ) obtained on the lattice, fixes the value of $\ell$ hence the energy unit.

\subsection{Critical temperature}

The thermodynamic quantities we have discussed so far, are dimensionless ratios, in units of the critical temperature. To compute $T_{c}$, we need an extra dimension-full quantity which can be used independently to set the unit of energy. In lattice studies this is typically the confining string tension $\sigma$ in the $T=0$ vacuum, with a value of around $(440 \mathrm{MeV})^{2}$, and results are given in terms of the dimensionless ratio $T_{c} / \sqrt{\sigma}$. In our case we cannot compute $\sigma$ directly, since it depends on the fundamental string tension, which is a priori unknown. Instead, we take the mass $m_{0++}$ of the lowest-lying glueball state as a reference.

masses. Only a precise knowledge of the underline string theory is expected to provide detailed information for such states. 
We compute $m_{0++}$ with the potential (4.1), with $V_{1}$ and $V_{3}$ fixed as in (5.3), then compare $T_{c} / m_{0++}$ to the same quantity obtained on the lattice. For the lattice result, we take the large $N_{c}$ result of [59], $T_{c} / \sqrt{\sigma}=0.5970(38)$, and combine it with the large $N_{c}$ result for the lowest-lying glueball mass [62], $m_{0++} / \sqrt{\sigma}=3.37(15)$. The two results are in fair agreement, without need to adjust any extra parameter:

$$
\left(\frac{T_{c}}{m_{0}}\right)_{h Q C D}=0.167, \quad\left(\frac{T_{c}}{m_{0}}\right)_{\text {lattice }}=0.177(7) .
$$

In physical units, the critical temperature we obtain is given by

$$
T_{c}=0.56 \sqrt{\sigma}=247 \mathrm{MeV}
$$

\subsection{String tension}

The fundamental string tension $T_{f}=\frac{1}{2 \pi \ell_{s}^{2}}$ cannot be computed from first principles in our model, but can be obtained using as extra input the lattice value of the confining string tension $\sigma$, at $T=0$. The fundamental and confining string tensions are related by eq. (4.10).

As for the critical temperature, we can relate $T_{f}$ to the value of the lowest-lying glueball mass, by using the lattice relation $\sqrt{\sigma}=\frac{m_{0++}}{3.37}$ [62]. Since what we actually compute numerically is $m_{0++} \ell$, this allows us to obtain the string tension $T_{f}$ (and fundamental string length $\ell_{s}=1 / \sqrt{2 \pi T_{f}}$ in AdS units:

$$
T_{f} \ell^{2}=0.19, \quad \ell_{s} / \ell=0.15
$$

This shows that the fundamental string length in our model is about an order of magnitude smaller than the AdS length. The meaning of this fact is a little more complicated conceptually, as the discussion in 15] indicates. Also, we should stress that, as discussed in Section 4 , this result depends on our choice of the overall normalization of $\lambda$ : changing the potential by $\lambda \rightarrow \kappa \lambda$ will yield different numerical values in (5.8) without affecting the other physical quantities.

Another related observable is the spatial string tension. It is calculated from the expectation value of the rectangular Wilson loop which stretches in spatial dimensions only. This has been calculated on the lattice [64], as well as using the high-temperature (resumed) perturbative expansion plus a zero-temperature calculation of the string tension in three-dimensional YM theory, 65]. The two calculation agree reasonably well.

The spatial string tension at finite temperature can be calculated in IHQCD, [66] by calculating the relevant Wilson loop. Very good agreement was found with the lattice calculations, especially at temperatures not far from the phase transition.

Finally, several calculations of quark-antiquark potentials exist. At zero temperature the long distance asymptotics of the quark potential was calculated in [14] and used to classify the dilaton potentials as a function of the confinement property. The full quark potential including the short distance behavior was computed in [67]. There a comparison to the Cornell potential was done as well as with quarkonium spectra finding excellent 
agreement with data. The issue of quarkonium potentials from IHQCD-like theories was also recently discussed in [68].

Finally the Polyakov loop was recently computed [69] in similar Einstein dilaton models that were studied first in [16].

\subsection{CP-odd sector}

The CP-odd sector of pure Yang-Mills is described holographically by the addition of a bulk pseudoscalar field $a(r)$ (the axion) with action: ${ }^{19}$

$$
S_{\text {axion }}=\frac{M_{p}^{3}}{2} \int d^{5} x Z(\lambda) \sqrt{-g}\left(\partial^{\mu} a\right)\left(\partial_{\mu} a\right)
$$

The field $a(r)$ is dual to the topological density operator $\operatorname{Tr} F \tilde{F}$. The prefactor $Z(\lambda)$ is a dilaton-dependent normalization. The axion action is suppressed by a factor $1 / N_{c}^{2}$ with respect to the action (2.1) for the dilaton and the metric, meaning that in the large- $N_{c}$ limit one can neglect the back-reaction of the axion on the background.

As shown in [14], requiring the correct scaling of $a(r)$ in the UV, and phenomenologically consistent axial glueball masses, constrain the asymptotics of $Z(\lambda)$ as follows:

$$
Z(\lambda) \sim Z_{0}, \lambda \rightarrow 0 ; \quad Z(\lambda) \sim \lambda^{4}, \lambda \rightarrow \infty
$$

where $Z_{0}$ is a constant. As a simple interpolating function between these large- and small$\lambda$ asymptotics we can take the following:

$$
Z(\lambda)=Z_{0}\left(1+c_{a} \lambda^{4}\right)
$$

The parameter $Z_{0}$ can be fixed by matching the topological susceptibility of pure YangMills theory, whereas $c_{a}$ can be fixed by looking at the axial glueball mass spectrum.

Axial glueballs. As in [14], we can fix $c_{a}$ by matching to the lattice results the mass ratio $m_{0-+} / m_{0++}$ between the lowest-lying axial and scalar glueball states. This is independent of the overall coefficient $Z_{0}$ in (5.11). The lattice value $m_{0-+} / m_{0++}=1.49$ [61] is obtained for:

$$
c_{a}=0.26 \text {. }
$$

With this choice, the mass of the first excited axial glueball state is in good agreement with the corresponding lattice result 61]:

$$
\left(\frac{m_{0-+*}}{m_{0++}}\right)_{h Q C D}=2.10 \quad\left(\frac{m_{0-+*}}{m_{0++}}\right)_{\text {lattice }}=2.12(10) .
$$

\footnotetext{
${ }^{19}$ This action was justified in $\sqrt{14}, \sqrt{15}$. The dilaton dependent coefficient $Z(\lambda)$ is encoding both the dilaton dependence as well as the UV curvature dependence of the axion kinetic terms in the associated string theory. We cannot determine it directly from the string theory, but we pin it down by a combination of first principles and lattice input, as we explain further below.
} 
Topological Susceptibility. In pure Yang-Mills, the topological $\chi$ susceptibility is defined by:

$$
E(\theta)=\frac{1}{2} \chi \theta^{2}
$$

where $E(\theta)$ is the vacuum energy density in presence of a $\theta$-parameter. $E(\theta)$ can be computed holographically by solving for the axion profile $a(r)$ on a given background, and evaluating the action (5.9) on-shell.

In the deconfined phase, the axion profile is trivial, implying a vanishing topological susceptibility [19]. This is in agreement with large- $N_{c}$ arguments and lattice results [21].

In the low-temperature phase, the axion acquires a non-trivial profile,

$$
a(r)=a_{U V} \frac{F(r)}{F(0)}, \quad F(r) \equiv \int_{r}^{\infty} \frac{d r}{Z(\lambda(r)) e^{3 A(r)}} .
$$

This profile is shown, for the case at hand, in Figure 10, where the axion is normalized to its UV value.

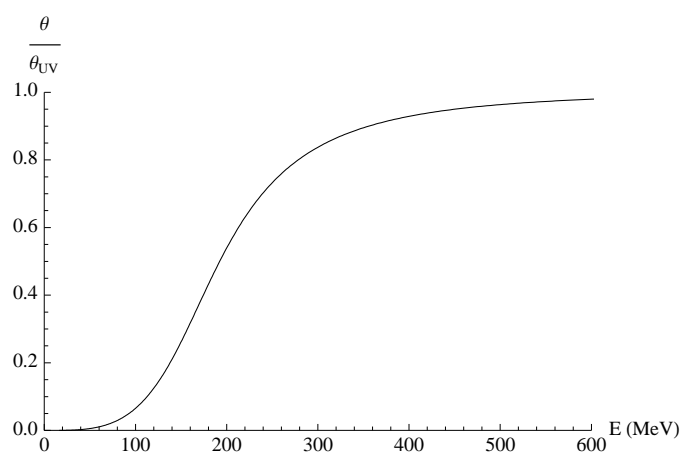

Figure 10: Axion profile in the radial direction. The $x$-axis is taken to be the energy scale, $E(r)=E_{0} b(r)$, where the unit $E_{0}$ is fixed to match the lowest glueball mass.

The topological susceptibility is given by [14]:

$$
\chi=M_{p}^{3} F(0)^{-1}=M_{p}^{3}\left[\int_{0}^{\infty} \frac{d r}{e^{3 A(r)} Z(r)}\right]^{-1},
$$

where $Z(r) \equiv Z(\lambda(r))$. Evaluating this expression numerically with $Z(\lambda)$ as in (5.11), and $c_{a}=0.26$ (to match the axial glueball spectrum ), we can determine the coefficient $Z_{0}$ by looking at the lattice result for $\chi$. For $N_{c}=3$, 70 obtained $\chi=(191 \mathrm{MeV})^{4}$, which requires $Z_{0}=133$.

In Table 4 we present a summary of the various physical quantities discussed in this section, as obtained in our holographic model, and their comparison with the lattice results for large $N_{c}$ (when available) and for $N_{c}=3$. The quantities shown in the upper half of the table are the ones that were used to fix the free parameters (reported in the last column) of the holographic model. 


\subsection{Coupling normalization}

Finally, we can relate the field $\lambda(r)$ to the running 't Hooft coupling. All other quantities we have discussed so far are scheme-independent and RG-invariant. This is not the case for the identification of the physical YM 't Hooft coupling, which is scheme dependent.

In the black-hole phase we can take $\lambda_{h} \equiv \lambda\left(r_{h}\right)$ as a measure of the temperaturedependent coupling. In figure 11 we show $\lambda_{h}$ as a function of the temperature in the range $T_{c}$ to $5 T_{c}$.

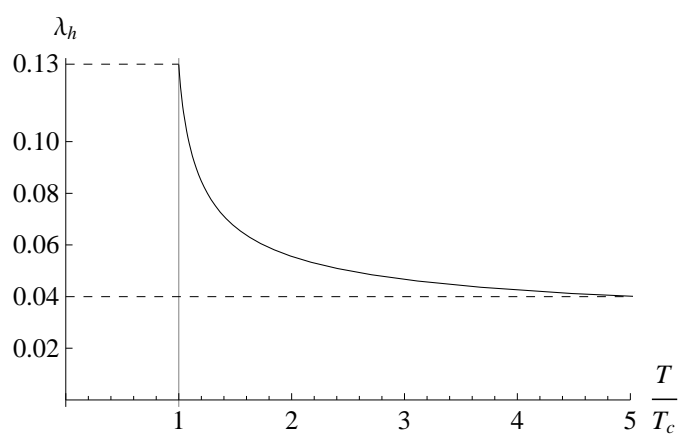

Figure 11: The coupling at the horizon as a function of temperature in the range $T_{c}-5 T_{c}$.

As a reference, we may take the result of [56], that found $g^{2}\left(5 T_{c}\right) \simeq 1.5$ for $N_{c}=3$, which translates to $\lambda_{t}\left(5 T_{c}\right) \simeq 5$. On the other hand, if we make the assumption that the identification $\lambda=\lambda_{t}$ is valid at all scales (not only in the UV), we find in our model $\lambda_{t}\left(5 T_{c}\right) \simeq 0.04$ (see Figure 11), i.e. a factor of 100 smaller than the lattice result.

This discrepancy is almost certainly due to the identification (4.2) being very different from lattice at strong coupling.

\section{Bulk viscosity}

The bulk viscosity $\zeta$ is an important probe of the quark-gluon plasma. Its profile as a function of $\mathrm{T}$ reveals information regarding the dynamics of the phase transition. In particular, both from the low-energy theorems and lattice studies [29, 30, 32], there is evidence that $\zeta$ increases near $T_{c}$.

For a viscous fluid the shear $\eta$ and bulk $\zeta$ viscosities are defined via the rate of entropy production as

$$
\frac{\partial s}{\partial t}=\frac{\eta}{T}\left[\partial_{i} v_{j}+\partial_{j} v_{i}-\frac{2}{3}(\partial \cdot v) \delta_{i j}\right]^{2}+\frac{\zeta}{T}(\partial \cdot v)^{2}
$$

Therefore, in a holographic setup, the bulk viscosity can be defined as the response of the diagonal spatial components of the stress-energy tensor to a small fluctuation of the metric. It can be directly related to the retarded Green's function of the stress-energy tensor by Kubo's linear response theory:

$$
\zeta=-\frac{1}{9} \lim _{\omega \rightarrow 0} \frac{1}{\omega} \operatorname{Im} G_{R}(\omega, 0),
$$


Table 2: Collected in this table is the complete set of physical quantities that we computed in our model and compared with data. The upper half of the table contains the quantities that we used as input (shown in boldface) for the holographic QCD model (HQCD). Each quantity can be roughly associated to one parameter of the model (last column). The lower half of the table contains our "postdictions" (i.e. quantities that we computed after all the parameters were fixed) and the comparison with the corresponding lattice results. The value we find for the critical temperature corresponds to $T_{c}=247 \mathrm{MeV}$.

\begin{tabular}{|c|c|c|c|c|}
\hline & HQCD & lattice $N_{c}=3$ & lattice $N_{c} \rightarrow \infty$ & Parameter \\
\hline$\left[p /\left(N_{c}^{2} T^{4}\right)\right]_{T=2 T_{c}}$ & 1.2 & 1.2 & - & $V 1=14$ \\
\hline$L_{h} /\left(N_{c}^{2} T_{c}^{4}\right)$ & 0.31 & 0.2856 & 0.3159 & $V 3=170$ \\
\hline$\left[p /\left(N_{c}^{2} T^{4}\right)\right]_{T \rightarrow+\infty}$ & $\pi^{2} / 45$ & $\pi^{2} / 45$ & $\pi^{2} / 45$ & $M_{p} \ell=\left[45 \pi^{2}\right]^{-1 / 3}$ \\
\hline$m_{0^{++}} / \sqrt{\sigma}$ & 3.37 & 3.5661 & 3.3762 & $\ell_{s} / \ell=0.15$ \\
\hline$m_{0^{-+}} / m_{0^{++}}$ & 1.49 & 1.4961 & - & $c_{a}=0.26$ \\
\hline$\chi$ & $(191 M e V)^{4}$ & $(191 M e V)^{4} 70$ & - & $Z_{0}=133$ \\
\hline$T_{c} / m_{0^{++}}$ & 0.167 & - & $0.177(7)$ & \\
\hline$m_{0^{*++}} / m_{0^{++}}$ & 1.61 & $1.56(11)$ & $1.90(17)$ & \\
\hline$m_{2^{++}} / m_{0^{++}}$ & 1.36 & $1.40(4)$ & $1.46(11)$ & \\
\hline$m_{0^{*-+}} / m_{0^{++}}$ & 2.10 & $2.12(10)$ & - & \\
\hline
\end{tabular}

where $G_{R}(w, \vec{p})$ is the Fourier transform of retarded Green's function of the stress-energy tensor:

$$
G_{R}(w, \vec{p})=-i \int d^{3} x d t e^{i \omega t-i \vec{p} \cdot \vec{x}} \theta(t) \sum_{i, j=1}^{3}\left\langle\left[T_{i i}(t, \vec{x}), T_{j j}(0,0)\right]\right\rangle
$$

A direct computation of the RHS on the lattice is non-trivial as it requires analytic continuation to Lorentzian space-time. In refs. [29], 30] the low energy theorems of QCD, as well 
as (equilibrium) lattice data at finite temperature were used in order to evaluate a particular moment of the spectral density of the relevant correlator. using a parametrization of the spectral density via two time-dependent constants, one of which is the bulk viscosity a relation for their product was obtained as a function of temperature. This can be converted to a relation for $\zeta$, assuming the other constant varies slowly with temperature.

The conclusion was that $\zeta / s$ increases near $T_{c}$. Another conclusion is that the fermionic contributions to $\zeta$ are small compared to the glue contributions.

The weak point of the approach of [30], is that it requires an ansatz on the spectrum of energy fluctuations, and further assumptions on the other parameters. which are not derived from first principles.

A direct lattice study of the bulk viscosity was also made in [32]. Here, the result is also qualitatively similar 12 . However, the systematic errors in this computation are large especially near $T_{c}$, mostly due to the analytic continuation that one has to perform after computing the Euclidean correlator on the lattice.

The results of references [29], 30] and the assumptions of the lattice calculation have been recently challenged in [71].

\subsection{The holographic computation}

The holographic approach offers a new way of computing the bulk viscosity. In the holographic set-up, $\zeta$ is obtained from (6.2). Using the standard AdS/CFT prescription, the two point-function of the energy-momentum tensor can be read off from the asymptotic behavior of the metric perturbations $\delta g_{\mu \nu}$. This is similar in spirit to the holographic computation of the shear viscosity [72], but it is technically more involved. A recent treatment of the fluctuation equation governing the scalar mode of a general Einstein-Dilaton system can be found in [73]. Here, we follow the method proposed by [74].

As explained in [74], one only needs to examine the equations of motion in the gauge $r=\Phi$, where the radial coordinate is equal to the dilaton. In our type of metrics, the applicability of this method requires some clarifications, that we provide in [76. Using $S O(3)$ invariance and the five remaining gauge degrees of freedom the metric perturbations can be diagonalized as

$$
\delta g=\operatorname{diag}\left(g_{00}, g_{11}, g_{11}, g_{11}, g_{55}\right)
$$

where

$$
\begin{gathered}
g_{00}=-e^{2 A} f\left[1+h_{00}(\varphi) e^{-i \varnothing t}\right], \quad g_{11}=e^{2 A}\left[1+h_{11}(\varphi) e^{-i \varnothing t}\right], \quad, \\
g_{55}=\frac{e^{2 B}}{f}\left[1+h_{55}(\varphi) e^{-i \varnothing t}\right],
\end{gathered}
$$

where the functions $A$ and $B$ emerge from the metric

$$
d s^{2}=e^{2 A(\varphi)}\left(-f d t^{2}+d x_{m} d x^{m}\right)+e^{2 B(\varphi)} \frac{d \varphi^{2}}{f} .
$$

Here, the fluctuations are taken to be harmonic functions of $t$ while having an arbitrary dependence on $\varphi$. 
The bulk viscosity depends only on the correlator of the diagonal components of the metric and so it suffices to look for the asymptotics of $h_{11}$. Interestingly, in the $r=\Phi$ gauge this decouples from the other components of the metric and satisfies the following equation $^{20}$

$$
h_{11}^{\prime \prime}-\left(-\frac{8}{9 A^{\prime}}-4 A^{\prime}+3 B^{\prime}-\frac{f^{\prime}}{f}\right) h_{11}^{\prime}-\left(-\frac{e^{2 B-2 A}}{f^{2}} \omega^{2}+\frac{4 f^{\prime}}{9 f A^{\prime}}-\frac{f^{\prime} B^{\prime}}{f}\right) h_{11}=0 .
$$

One needs to impose two boundary conditions. First, we require that only the infalling condition survives at the horizon:

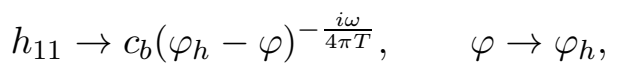

where $c_{b}$ is a normalization factor. The second boundary condition is that $h_{11}$ has unit normalization on the boundary:

$$
h_{11} \rightarrow 1, \quad \varphi \rightarrow-\infty
$$

Having solved for $h_{11}(\varphi)$, Kubo's formula (6.2) and a wise use of the AdS/CFT prescription to compute the stress-energy correlation function [74] determines the ratio of bulk viscosity as follows.

The AdS/CFT prescription relates the imaginary part of the retarded $T_{i i}$ Green's function to the number flux of the $h_{11}$ gravitons $\mathcal{F}$ [74]:

$$
\operatorname{Im} G_{R}(\omega, 0)=-\frac{\mathcal{F}}{16 \pi G_{5}}
$$

where the flux can be calculated as the Noether current associated to the $U(1)$ symmetry $h_{11} \rightarrow e^{i \theta} h_{11}$ in the gravitational action for fluctuations. One finds,

$$
\mathcal{F}=i \frac{e^{4 A-B} f}{3 A^{\prime 2}}\left[h_{11}^{*} h_{11}^{\prime}-h_{11} h_{11}^{*^{\prime}}\right]
$$

As $\mathcal{F}$ is independent of the radial variable, one can compute it at any $\varphi$, most easily near the horizon, where $h_{11}$ takes the form (6.8). Using also the fact that $(d A / d \varphi)\left(\varphi_{h}\right)=$ $-8 V\left(\varphi_{h}\right) / 9 V^{\prime}\left(\varphi_{h}\right)$, one finds

$$
\mathcal{F}(\varnothing)=\frac{27}{32} \varnothing\left|c_{b}(\varnothing)\right|^{2} e^{3 A\left(\varphi_{h}\right)} \frac{V^{\prime}\left(\varphi_{h}\right)^{2}}{V\left(\varphi_{h}\right)} .
$$

Then, (6.2) and (6.10) determine the ratio of bulk viscosity and the entropy density as,

$$
\frac{\zeta}{s}=\frac{3}{32 \pi}\left(\frac{V^{\prime}\left(\varphi_{h}\right)}{V\left(\varphi_{h}\right)}\right)^{2}\left|c_{b}\right|^{2} .
$$

In the derivation we use the Bekenstein-Hawking formula for the entropy density, $s=$ $\exp 3 A\left(\varphi_{h}\right) / 4 G_{5}$.

To find $\zeta$ we need to find $c_{b}$ only in the limit $\omega \rightarrow 0$. The computation is performed by numerically solving equation (6.7) with the appropriate boundary conditions. There are two separate methods that one can employ to determine the quantity $c_{b}$ :

\footnotetext{
${ }^{20}$ Difference in the various numerical factors in this equation w.r.t 74 is due to our different normalization of the dilaton kinetic term.
} 


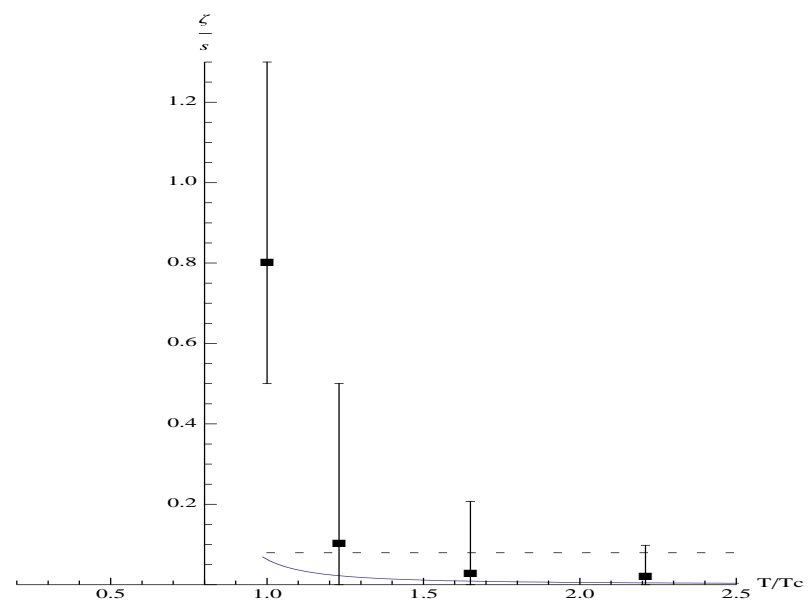

Figure 12: Plot of $\zeta / s$ (continuous line) calculated in Improved Holographic QCD model. This is compared with the lattice data of 32 that are shown as boxes. The horizontal dashed line is indicating the (universal) value of $\frac{\eta}{s}$ for comparison.

1. One can solve (6.7) numerically with a fixed $\omega / T$, but small enough so that $c_{b}$ reaches a fixed value. The method is valid also for finite values of $\omega$. From a practical point of view, it is easier to solve (6.7) with the boundary condition (6.8) with a unit normalization factor, read off the value on the boundary $h_{11}(-\infty)$ from the solution and finally use the symmetry of (6.7) under constant scalings of $h_{11}$ to determine $\left|c_{b}\right|=1 /\left|h_{11}(-\infty)\right|$.

2. An alternative method of computation that directly extracts the information at $\omega=0$ follows from the following trick [74. Instead of solving (6.7) for small but finite $\omega$, one can instead solve it for $\omega=0$. This is a simpler equation, yet complicated enough to still evade analytic solution. Let us call this solution $h_{11}^{0}$. One numerically solves it by fixing the boundary conditions on the boundary: $h_{11}^{0}(-\infty)=1$ and the derivative $d h_{11}^{0} / d \varphi(-\infty)$ is chosen such that $h_{11}$ is regular at the horizon. Matching this solution to the expansion of (6.8) for small $\omega$ than yields $\left|c_{b}\right|=h_{11}^{0}\left(\varphi_{h}\right)$.

Both methods were used to obtain $\zeta / s$ as a function of $\mathrm{T}$ and checked that they yield the same result. As explained in [19], most of the thermodynamic observables are easily computed using the method of scalar variables, [19, 76].

The results are presented in figure 12. This figure gives a comparison of the curve obtained by the holographic calculation sketched above by solving (6.7) and the lattice data of [32]. We also show $\eta / s=1 / 4 \pi$ in this figure for comparison. The result is qualitatively similar to the lattice result where $\zeta / s$ increases as $T$ approaches $T_{c}$, however the rate of increase is slower than the lattice. As a result, we obtain a value $\zeta / s\left(T_{c}\right) \approx 0.06$ that is an order of magnitude smaller than the lattice result 32 which is 0.8. Note however that the error bars in the lattice evaluation are large near $T_{c}$ and do not include all possible systematic errors from the analytic continuation. 
We should note the fact that the holographic calculation gives a smaller value for the bulk viscosity near $T_{c}$ than the lattice calculation is generic and has been found for other potentials with similar IR asymptotics, [74]. The fact that the value of $\zeta / s$ near $T_{c}$ is correlated with the IR asymptotics of the potential will be shown further below.

Another fact that one observes from figure 12 is that $\zeta / s$ vanishes in the high $T$ limit. This reflects the conformal invariance in the UV and can be shown analytically as follows. $\zeta / s$ is determined by formula (6.13). In the high $\mathrm{T}$ limit, (corresponding to $\lambda_{h} \rightarrow 0$, near the boundary), the fluctuation coefficient $\left|c_{b}\right| \rightarrow 1$. This is because of the boundary condition $h_{11}(\lambda=0)=1$. We use the relation between $\mathrm{T}$ and $\lambda_{h}$ in the high $\mathrm{T}$ limit [19],

$$
\lambda_{h} \rightarrow\left(\beta_{0} \log (\pi T / \Lambda)\right)^{-1} .
$$

Substitution in (6.13) leads to the result,

$$
\left.\frac{\zeta}{s}\right|_{\text {large }} \rightarrow \frac{2}{27 \pi} \frac{1}{\log ^{2}(\pi T / \Lambda)}, \quad \text { as } T \rightarrow \infty .
$$

As $s$ itself diverges as $T^{3}$ in this limit - it corresponds to an ideal gas - we learn that $\zeta$ also diverges as $T^{3} / \log ^{2}(T)$. Divergence at high $\mathrm{T}$ is expected from the bulk-viscosity of an ideal gas. We do not expect however the details of the asymptotic result to match with the pQCD result, for the same reasons that the shear-viscosity-to-entropy ratio does not, 15. We note however, that the asymptotic T-dependence is very similar to the pQCD result, 75 :

$$
\zeta / s \propto \log ^{-2}(\pi T / \Lambda) \log ^{-1} \log (\pi T / \Lambda) .
$$

\subsection{Holographic explanation for the rise of $\zeta / s$ near $T_{c}$ and the small black-hole branch}

With the same numerical methods, one can also compute the ratio $\zeta / s$ on the small blackhole branch. As this solution has a smaller value of the action than the large black-hole solution, it is a subleading saddle point in the phase space of the theory, hence bears no direct significance for an holographic investigation of the quark-gluon plasma. However, as we show below, the existence of this branch provides a holographic explanation for the peak in $\zeta / s$ in the quark-gluon plasma, near $T_{c}$.

From the practical point of view, we find the second numerical method above (solving the fluctuation equation at $\omega=0$ ) easier in the range of $\lambda_{h}$ that corresponds to the small black hole. The result is shown in figure 13 (a). The presence of two branches for $T>T_{\min }$, is made clear in this figure. See also fig 13 (b) for the respective ranges of $\lambda_{h}$ that correspond to small and large BHs. In fig 13 (a), $\zeta / s$ on the large BH branch is depicted with a solid curve and the small $\mathrm{BH}$ branch is depicted with a dashed curve. We observe that $\zeta / s$ keeps increasing on the large- $\mathrm{BH}$ branch as $\mathrm{T}$ is lowered, up to the temperature $T_{\min }$ where the small and large $\mathrm{BH}$ branches merge ${ }^{21}$. On the other hand, on the small $\mathrm{BH}$ branch $\zeta / s$ keeps increasing as the $\mathrm{T}$ is increased, up to a certain $T_{\max }$ that lies between $T_{\min }$ and $T_{c}$, see figure 14. From this point onwards, $\zeta / s$ decreases with increasing T.

\footnotetext{
${ }^{21} \mathrm{As}$ far as the thermodynamics of the gluon plasma is concerned, the temperatures below $T_{c}$ (on the large $\mathrm{BH}$ branch) has little importance, because for $T<T_{c}$ the plasma is in the confined phase.
} 


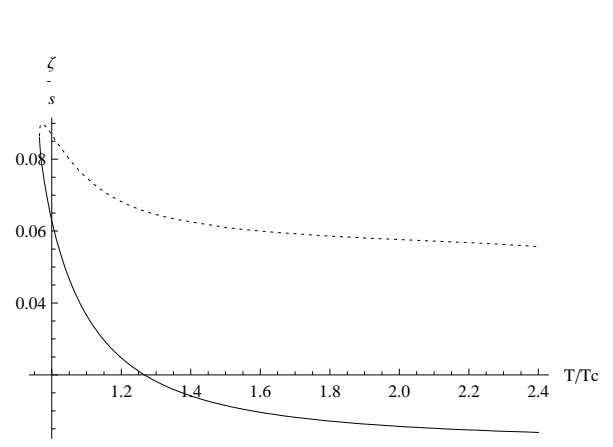

(a)

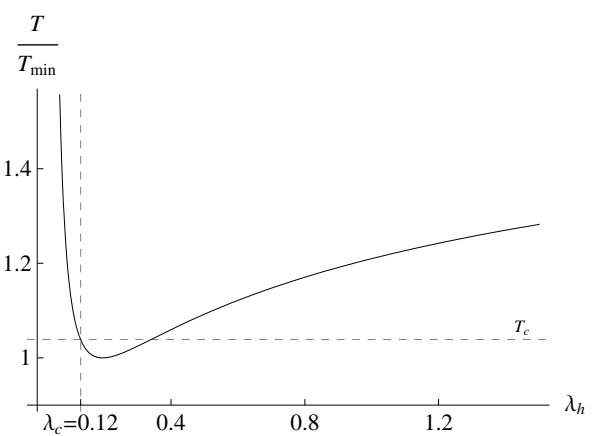

(b)

Figure 13: (a) Numerical evaluation of $\zeta / \eta$ both on the large-BH branch (the solid curve) and on the small $\mathrm{BH}$ branch (the dashed curve). $T_{m}$ denotes $T_{\min }$. (b) The two branches of black-hole solutions, that correspond to different ranges of $\lambda_{h}$. The large $\mathrm{BH}$ corresponds to $\lambda_{h}<\lambda_{\min }$ and the small BH corresponds to $\lambda_{h}>\lambda_{\text {min }}$.

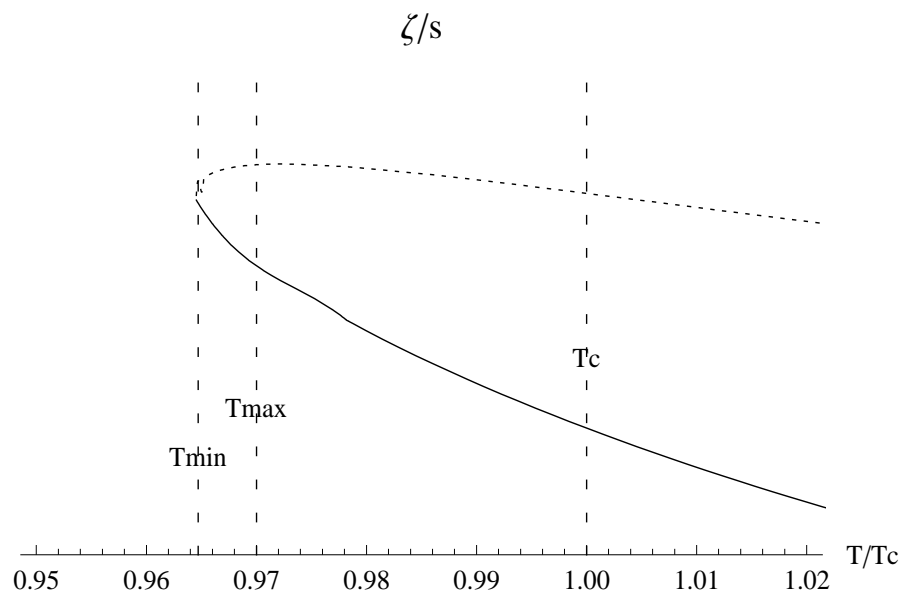

Figure 14: An inset from the figure 13 around the maximum of $\zeta / s$.

A simple fact that can be proved analytically is that the derivative of $\zeta / s$ diverges at $T_{\text {min }}$. This is also clear from figure 14. This is shown by inspecting equation (6.13). The $\mathrm{T}$ derivative is determined as $d / d T=\left(d T / d \lambda_{h}\right) d / d \lambda_{h}$. Whereas the derivative w.r.t $\lambda_{h}$ is everywhere smooth ${ }^{22}$, the factor $d T / d \lambda_{h}$ diverges at $T_{\text {min }}$ by definition, see figure 13 (b).

Therefore, the presence of a $T_{\min }$ where the large and the small black holes meet, in other words, the presence of a small black-hole branch is responsible for the increase of $\zeta / s$ near $T_{\text {min }}$. As in most of the holographic constructions that we analyzed, and specifically in the example we present here, $T_{c}$ and $T_{\min }$ are close to one another, this fact implies a rise in the bulk viscosity near $T_{c}$. This proposal, combined with the fact that the existence of a small BH branch and color confinement in the dual gauge theory at zero $T$ are in one-

\footnotetext{
${ }^{22}$ Note that $c_{b}$ is also a function of $\lambda_{h}$. As both the fluctuation equation (6.7) and the boundary conditions are smooth at $\lambda_{h}=\lambda_{\min }$, one concludes that $c_{b}$ also is smooth at this point.
} 
to-one correspondence [19], suggests that in confining large- $N$ gauge theories, there will be a peak in the ratio $\zeta / s$ close to $T_{c}$.

Another fact that can be shown analytically is that $\zeta / s$ asymptotes to a finite value as $T \rightarrow \infty$ in the small black-hole branch ${ }^{23}$. We find that,

$$
\left.\frac{\zeta}{s}\right|_{\text {small }} \rightarrow \frac{1}{6 \pi}, \quad \text { as } T \rightarrow \infty
$$

As the entropy density vanishes in this limit [19], we conclude that $\zeta$ should vanish with the same rate.

For a general potential with strong coupling asymptotics

$$
V(\lambda) \sim \lambda^{Q} \quad \text { as } \quad \lambda \rightarrow \infty
$$

taking into account (6.13), equation (6.17) is modified to

$$
\left.\frac{\zeta}{s}\right|_{\text {small }} \rightarrow \frac{3 Q^{2}}{32 \pi}, \quad \text { as } r_{h} \rightarrow r_{0} .
$$

where $r_{0}$ is the position of the singularity in the zero temperature solution.

For confining theories, the limit $r_{h} \rightarrow r_{0}$ corresponds to $T \rightarrow \infty$ on the small $\mathrm{BH}$ branch. However, one can show that the result $(6.19)$ holds quite generally, regardless of whether the zero $\mathrm{T}$ theory confines or not. In particular, for the non-confining theoriesthat is either when $Q<4 / 3$ or when $Q=4 / 3$ but the subleading term in the potential vanishes at the singularity - there is only the large black-hole branch and the limit $r_{h} \rightarrow r_{0}$ corresponds to the zero $\mathrm{T}$ limit of this $\mathrm{BH}$. Thus, we also learn that there exist holographic models that correspond to non-confining gauge theories whose zero $\mathrm{T}$ limit yield a constant $\zeta / s$. This constant approaches zero as $Q \rightarrow 0$, i.e. in the limiting AdS case.

We also see that the asymptotic value of $\zeta / s$ in the small $\mathrm{BH}$ branch is close to the value of $\zeta / s$ near $T_{c}$. We shall give an explanation of this fact in the next subsection. Using the asymptotic formula (6.19), the fact that $Q>\frac{4}{3}$ for confinement and $Q \leq \frac{4 \sqrt{2}}{3}$ for the IR singularity to be good and repulsive we may obtain a range of values where we expect $\zeta / s$ to vary, namely

$$
\frac{1}{6 \pi} \leq\left.\frac{\zeta}{s}\right|_{\text {small,asymptotic }} \leq \frac{1}{3 \pi}
$$

A final observation concerns the coefficient $c_{b}\left(\lambda_{h}\right)$ in (6.13). This part is the only input from the solution of the fluctuation equation, the rest of $(6.13)$ is fixed by the dilaton potential entirely. We plot the numerical result for $c_{b}$ in fig 15 as a function of the coupling at the horizon $\lambda_{h}$.

First of all, Figure 15 provides a check that, the approximate bound of [74] $\left|c_{b}\right| \geq 1$, is satisfied in the entire range. One also observes $c_{b}$ approaches to 1 in the IR and UV asymptotics. These facts can be understood analytically: In the UV (near the boundary) it is because of the boundary condition $c_{b}=1$. In the IR, it is more subtle, and it is explained in appendix B of [76].

\footnotetext{
${ }^{23}$ See the discussion at appendix $\mathrm{B}$ of $[76$.
} 


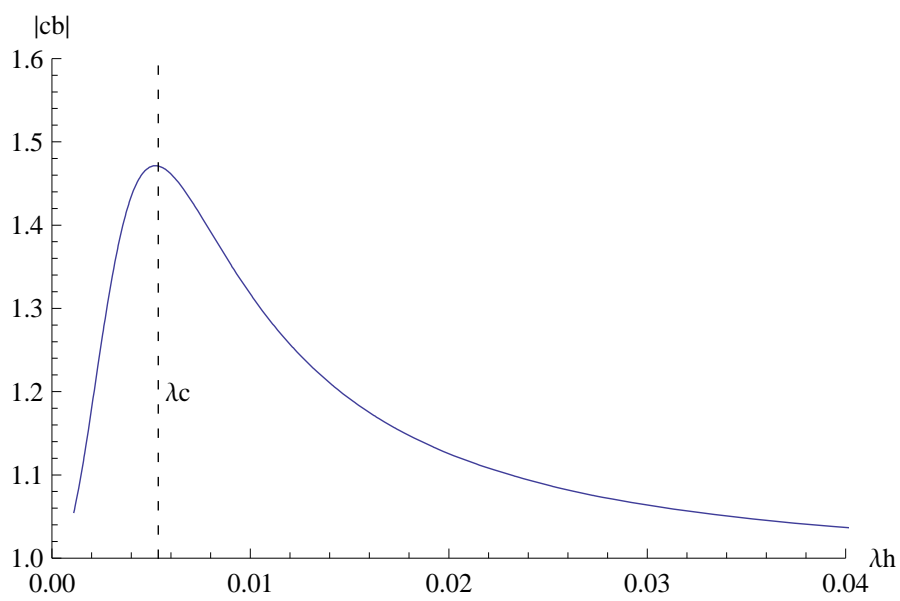

Figure 15: The coefficient $\left|c_{b}\right|$ of equation (6.13) as a function of $\lambda_{h}$.

Finally, we observe that the deviation of $c_{b}$ from the asymptotic value 1 is maximum around the phase transition point $\lambda_{c}$. In fact, we numerically observed that the top of the curve in figure 15 coincides with $\lambda_{c}$ to a very high accuracy. Whether this is just a coincidence or not, remains to be clarified.

\subsection{The adiabatic approximation}

Motivated by the Chamblin-Reall solutions [77], Gubser et al. [16] proposed an approximate adiabatic formula for the speed of sound. In the case when $V^{\prime} / V$ is a slowly varying function of $\varphi$, [16] proposes the following formulae for the entropy density and the temperature:

$$
\begin{aligned}
\log s & =-\frac{8}{3} \int^{\varphi_{h}} d \varphi \frac{V}{V^{\prime}}+\cdots, \\
\log T & =\int^{\varphi_{h}} d \varphi\left(\frac{1}{2} \frac{V^{\prime}}{V}-\frac{8}{9} \frac{V}{V^{\prime}}\right) \cdots,
\end{aligned}
$$

where the ellipsis denote contributions slowly varying in $\varphi_{h}{ }^{24}$.

It is very useful to reformulate this approximation using the method of scalar variables, which in turn allows us to extract the general $\mathrm{T}$ dependence of most of the thermodynamic observables in an approximate form. Here, we apply this formalism to the computation of $\zeta / s$. The method of scalar variables and the details of the adiabatic approximation in the scalar variables are given in [76].

For the scalar variable $X=\frac{\Phi^{\prime}}{3 A^{\prime}}$ the adiabatic approximation means

$$
X(\varphi) \approx-\frac{3}{8} \frac{V^{\prime}(\varphi)}{V(\varphi)}
$$

The fluctuation equation (6.7) greatly simplifies with (6.23). In fact, as shown in [76], the solution becomes independent of $\varphi$. With unit normalization on boundary, the adiabatic

\footnotetext{
${ }^{24}$ Various coefficients in these equations differ from [16] due to our different normalization of the dilaton kinetic term.
} 


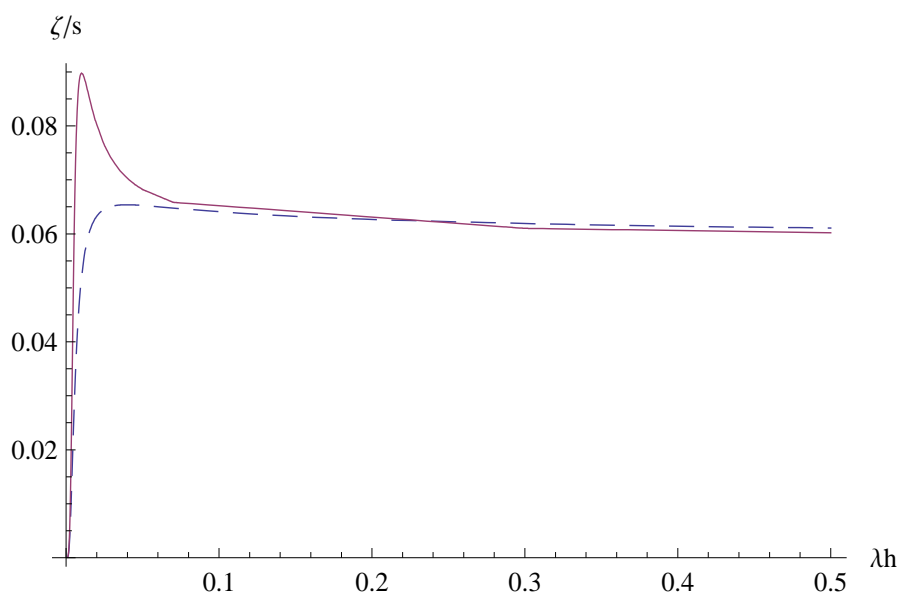

Figure 16: Comparison of the exact $\zeta / s$ with the adiabatic approximation in the variable $\lambda_{h}$. Solid(red) curve is the full numerical result and the dashed(blue) curve follows from (6.24).

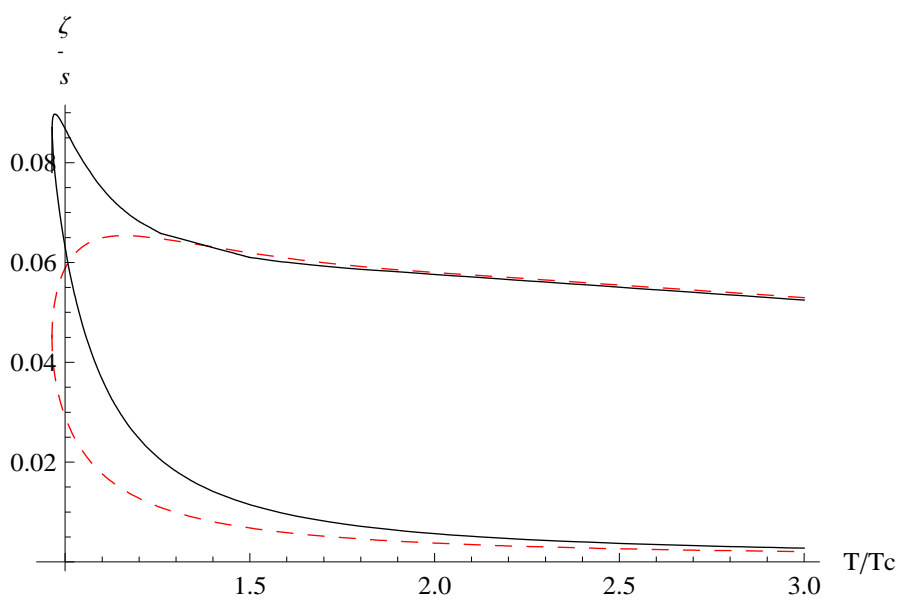

Figure 17: Comparison of the exact $\zeta / s$ with the adiabatic approximation in variable T. Solid(blue) curve is the full numerical result and the dashed(red) curve follows from (6.24).

solution in the entire range of $\varphi \in\left(-\infty, \varphi_{h}\right)$ becomes $h_{a d b}(\varphi)=1$. Consequently, the coefficient $c_{b}$ in $(6.13)$ becomes unity, hence:

$$
\left.\frac{\zeta}{s}\right|_{a d b}=\frac{3}{32 \pi}\left(\frac{V^{\prime}\left(\varphi_{h}\right)}{V\left(\varphi_{h}\right)}\right)^{2} .
$$

We plot this function in $\lambda_{h}$ in figure 16, where we also provide the exact numerical result for comparison. Note that in figure 16 the whole large black-hole branch has been compressed at the left of the figure for $\lambda_{h} \lesssim 0.04$ The same functions in the variable $T / T_{c}$ are plotted in figure 17 .

The validity of the adiabatic approximation equation $(6.23)$, is determined by the rate at which $V^{\prime} / V$ varies with $\Phi$. In particular, the approximation becomes exact in the limits 
where $V^{\prime} / V$ becomes constant. This happens for a constant potential or a potential that is a single power of $\lambda$ (exponential in $\Phi)$. This is the case in the UV $(\varphi \rightarrow-\infty$, where the potential becomes a constant) and the IR $(\varphi \rightarrow+\infty$ where the potential becomes a power law.) . Therefore equation (6.24) allows us to extract the analytic behavior of $\zeta / s$ in the limits $\Phi_{h} \rightarrow \pm \infty$.

The numerical values one obtains from (6.24) in the intermediate region may differ from the exact result (6.13) considerably, especially near $T_{c}$. However, we expect that the general shape will be similar.

Finally, the adiabatic approximation hints at why, in the particular background that we study, $\zeta / s$ at $T_{c}$ is close to the limit value (6.17): In order to see this we rewrite (6.24) as

$$
\left.\frac{\zeta}{s}\right|_{a d b}=\frac{2}{3 \pi} X^{2} \text {. }
$$

In the limit (6.17) we have $X \rightarrow-1 / 2$. The only other point where $X=-1 / 2$, is at the minimum of the string frame scale factor $\varphi_{*}$. This is the point where the confining string saturates [14]. On the other hand, we expect on general physical grounds that the de-confinement phase transition happens near this point, i.e. $\varphi_{c} \approx \varphi_{*}$. Thus, the adiabatic formula predicts that $\zeta / s\left(\varphi_{c}\right)$ be close to the limit value $1 / 6 \pi .^{25}$

\subsection{Buchel's bound}

In [78], Buchel proposed a bound for the ratio of the bulk and shear viscosities, motivated by certain well-understood holographic examples. In 4 space-time dimensions the Buchel bound reads,

$$
\frac{\zeta}{\eta} \geq 2\left(\frac{1}{3}-c_{s}^{2}\right)
$$

We note that the bound is proposed to hold in the entire range of temperature from $T_{c}$ to $\infty$. This bound is trivially satisfied for exact conformal theories such as $\mathcal{N}=4 \mathrm{YM}$, and saturated in theories on $D p$ branes [78, 79]. With the numerical evaluation at hand, we can check (6.26) in our case. In figure 18 (a) we plot the LHS and RHS of the bound ${ }^{26}$. We clearly see that the bound is satisfied for all temperatures. As expected, both the LHS and the RHS of (6.26) vanishes in the high $\mathrm{T}$ conformal limit.

A clear picture of Buchel's bound is obtained by defining the function:

$$
C(T)=\frac{\zeta / \eta}{2\left(1 / 3-c_{s}^{2}\right)}
$$

in terms of which the bound is simply $C>1$. In Figure 18 (b) we show the function $C(T)$ obtained numerically in our IHQCD model, between $T_{c}$ and $5 T_{c}$. The values of this function are mildly dependent on temperature, and are between 1.5 and 2 , the same range of values that were recently considered in the hydrodynamic codes by Heinz and Song [80].

\footnotetext{
${ }^{25}$ This argument may break down for two (dependent) reasons: First of all the adiabatic approximation becomes lees good near $\varphi_{c}$. This is because, in this region $V^{\prime} / V$ varies relatively more rapidly as a function of $\varphi$. Secondly, precisely because of this, even though $\varphi_{c}$ is not far away from $\varphi_{*}$ the difference can result in a considerable change in the value of $\zeta / s$ through (6.24).

${ }^{26}$ Since this theory contains two derivatives only, $\frac{\eta}{s}$ has the universal value $1 / 4 \pi$.
} 


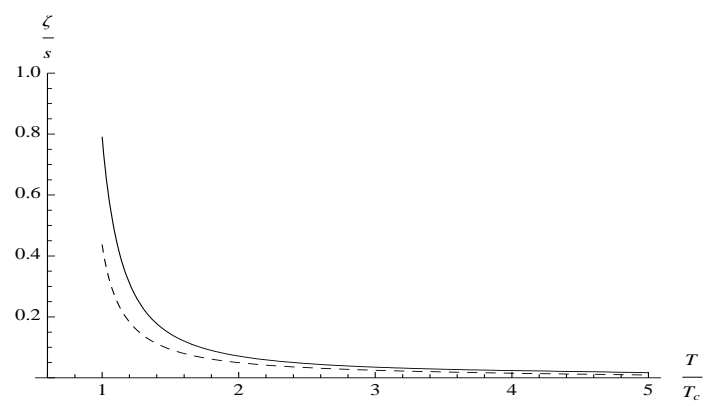

(a)

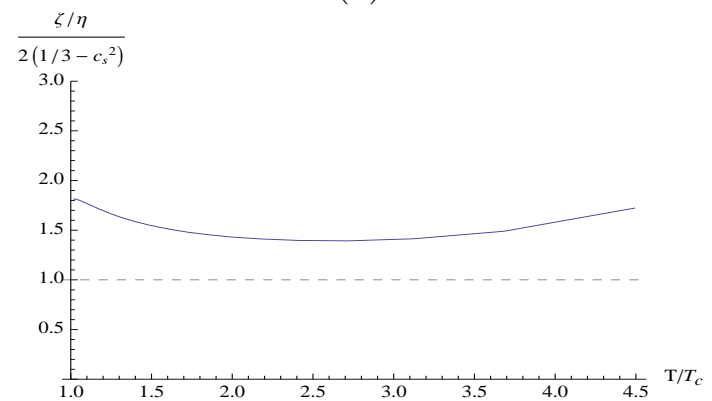

(b)

Figure 18: (a) Comparison of $\zeta / \eta$ (solid line) and the RHS of (6.26) (dashed line), obtained using the speed of sound of the IHQCD model 22]. (b) Plot of the function $C(T)$ defined in equation (6.27) as a function of temperature. The horizontal dashed line indicates where Buchel's bound is saturated. We see that the bound is satisfied in the entire range of temperatures.

We may also investigate the fate of the bound at large T. using the asymptotics of $\zeta / s$ in 6.15

$$
\left.\frac{\zeta}{s}\right|_{\text {large }}=\frac{2}{27 \pi} \frac{1}{\log ^{2}(\pi T / \Lambda)}+\cdots, \quad \text { as } T \rightarrow \infty .
$$

and

$$
\frac{1}{c_{s}^{2}}-3=\frac{4}{3} \frac{1}{\log ^{2}\left(\frac{T}{T_{c}}\right)}+\frac{32 b}{9} \frac{\log \left(\log \left(\frac{T}{T_{c}}\right)\right)}{\log ^{3}\left(\frac{T}{T_{c}}\right)}+\cdots
$$

from [19], that can be rewritten as

$$
\frac{1}{3}-c_{s}^{2}=\frac{4}{27} \frac{1}{\log ^{2}\left(\frac{T}{T_{c}}\right)}+\frac{32 b}{81} \frac{\log \left(\log \left(\frac{T}{T_{c}}\right)\right)}{\log ^{3}\left(\frac{T}{T_{c}}\right)}+\cdots
$$

where $b=\frac{b_{1}}{b_{0}^{2}}=\frac{3 \cdot 34}{2 \cdot 121}$ is the ratio of the two-loop to the one-loop squared $\beta$-function coefficients in large- $N_{c}$ YM.

Since in this class of models $\eta / s=1 / 4 \pi$ exactly we obtain

$$
\lim _{T \rightarrow \infty} \frac{\zeta / \eta}{2\left(1 / 3-c_{s}^{2}\right)}=1
$$


in agreement with a recently derived general formula, in Einstein dilaton gravity, [81,

$$
\lim _{T \rightarrow \infty} \frac{\zeta / \eta}{2\left(1 / 3-c_{s}^{2}\right)}=2 \pi \frac{4-\Delta}{4-2 \Delta} \cot \left(\frac{\pi \Delta}{4}\right)
$$

where $\Delta$ is the scaling dimensions of the scalar operator in the UV, that is marginal in our case.

It has also been suggested recently, [82, 83], that the speed of sound squared, in Einstein dilaton gravity asymptotes to $1 / 3$ at high temperatures from below. This is evident in our asymptotic formula $(6.30)$, although the formulae in [82, 83] fail to capture correctly the marginal case that is relevant here.

\section{The drag force on strings and heavy quarks}

We will now consider an (external) heavy quark moving through an infinite volume of gluon plasma with a fixed velocity $v$ at a finite temperature $\mathrm{T}$ [9, 11]. The quark feels a drag force coming from its interaction with the plasma and an external force has to be applied in order for it to keep a constant velocity. In a more realistic set up one would like to describe the deceleration caused by the drag.

The heavy external quark can be described by a string whose endpoint is at the boundary. One can accommodate flavor by introducing D-branes, but we will not do this here. A first step is to describe the classical string "trailing" the quark.

We consider the Nambu-Goto action on the world-sheet of the string.

$$
S_{N G}=-\frac{1}{2 \pi \ell_{s}^{2}} \int d \sigma d \tau \sqrt{\operatorname{det}\left(-g_{M N} \partial_{\alpha} X^{M} \partial_{\beta} X^{N}\right)},
$$

where the metric is the string frame metric. The ansatz we are going to use to describe the trailing string is, 11,

$$
X^{1}=v t+\xi(r), \quad X^{2}=X^{3}=0,
$$

along with the gauge choice

$$
\sigma=r, \quad \tau=t
$$

where $r$ is the (radial) holographic coordinate. The string is moving in the $X^{1}$ direction.

This is a "steady-state" description of the moving quark as acceleration and deceleration are not taken into account. For a generic background the action of the string becomes

$$
S=-\frac{1}{2 \pi \ell_{s}^{2}} \int d t d r \sqrt{-g_{00} g_{r r}-g_{00} g_{11} \xi^{\prime 2}-g_{11} g_{r r} v^{2}} .
$$

Note that $g_{00}$ is negative, and we should check whether our solution produces a real action. For example a straight string stretching from the quark to the horizon is a solution to the equations of motion but has imaginary action.

We note that the action does not depend on $\xi$ but only its derivative, therefore the corresponding "momentum" is conserved

$$
\pi_{\xi}=-\frac{1}{2 \pi \ell_{s}^{2}} \frac{g_{00} g_{11} \xi^{\prime}}{\sqrt{-g_{00} g_{r r}-g_{00} g_{11} \xi^{\prime 2}-g_{11} g_{r r} v^{2}}} .
$$


We solve for $\xi^{\prime}$ to obtain

$$
\xi^{\prime}=\frac{\sqrt{-g_{00} g_{r r}-g_{11} g_{r r} v^{2}}}{\sqrt{g_{00} g_{11}\left(1+g_{00} g_{11} /\left(2 \pi \ell_{s}^{2} \pi_{\xi}\right)^{2}\right)}} .
$$

The numerator changes sign at some finite value of the fifth coordinate $r_{s}$. For the solution to be real, the denominator has to change sign at the same point. We therefore determine $r_{s}$ via the equation

$$
g_{00}\left(r_{s}\right)+g_{11}\left(r_{s}\right) v^{2}=0
$$

and the constant momentum

$$
\pi_{\xi}^{2}=-\frac{g_{00}\left(r_{s}\right) g_{11}\left(r_{s}\right)}{\left(2 \pi \ell_{s}^{2}\right)^{2}}
$$

Writing the string-frame metric as

$$
d s^{2}=e^{2 A_{s}}\left[\frac{d r^{2}}{f}-f d t^{2}+d x \cdot d x\right]
$$

(7.7) becomes

$$
v^{2}=f\left(r_{s}\right)
$$

The induced world-sheet metric is therefore

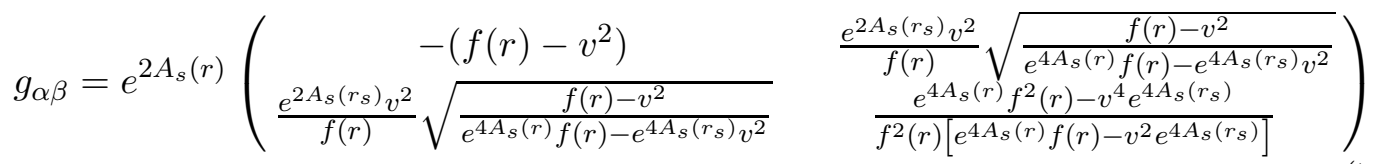

We can change the time coordinate to obtain a diagonal induced metric $t=\tau+\zeta(r)$ with

$$
\zeta^{\prime}=\frac{e^{2 A_{s}\left(r_{s}\right)} v^{2}}{f(r) \sqrt{\left(f(r)-v^{2}\right)\left(e^{4 A_{s}(r)} f(r)-e^{4 A_{s}\left(r_{s}\right)} v^{2}\right)}}
$$

The new metric is

$$
d s^{2}=e^{2 A_{s}(r)}\left[-\left(f(r)-v^{2}\right) d \tau^{2}+\frac{e^{4 A_{s}(r)}}{\left(e^{4 A_{s}(r)} f(r)-e^{4 A_{s}\left(r_{s}\right)} v^{2}\right)} d r^{2}\right]
$$

and near $r=r_{s}$ it has the expansion

$d s^{2}=\left[-f^{\prime}\left(r_{s}\right) e^{2 A_{s}\left(r_{s}\right)}\left(r-r_{s}\right)+\mathcal{O}\left(\left(r-r_{s}\right)^{2}\right)\right] d \tau^{2}+\left[\frac{e^{2 A_{s}\left(r_{s}\right)}}{\left(4 v^{2} A_{s}^{\prime}\left(r_{s}\right)+f^{\prime}\left(r_{s}\right)\right)\left(r-r_{s}\right)}+\mathcal{O}(1)\right] d r^{2}$

This is a world-sheet black-hole metric with horizon at the turning point $r=r_{s}$.

\subsection{The drag force}

The drag force on the quark can be determined by calculating the momentum that is lost by flowing along the string into the horizon:

$$
F_{\text {drag }}=\frac{d p_{1}}{d t}=-\frac{1}{2 \pi \ell_{s}^{2}} \frac{g_{00} g_{11} \xi^{\prime}}{\sqrt{-g}}=\pi_{\xi} .
$$


This can be obtained by considering the world-sheet Noether currents $\Pi_{M}^{\alpha}$ and expressing the loss of momentum as $\Delta P_{x_{1}}^{z}=\int \Pi_{1}^{r}$. This may be evaluated at any value of $\mathrm{r}$, but it is more convenient to evaluate it at $r=r_{s}$.

We finally find that

$$
F_{\text {drag }}=-\frac{1}{2 \pi \ell_{s}^{2}} \sqrt{-g_{00}\left(r_{s}\right) g_{11}\left(r_{s}\right)}
$$

Using the form (7.9) of our finite-temperature metric in the string frame we finally obtain

$$
F_{\text {drag }}=-\frac{e^{2 A_{s}\left(r_{s}\right)} \sqrt{f\left(r_{s}\right)}}{2 \pi \ell_{s}^{2}}=-\frac{e^{2 A\left(r_{s}\right)} \sqrt{f\left(r_{s}\right)} \lambda\left(r_{s}\right)^{4 / 3}}{2 \pi \ell_{s}^{2}},
$$

where in the second equality we expressed the force in terms of the Einstein-frame scale factor and the "running" dilaton. Substituting from (7.10) we obtain

$$
F_{\text {drag }}=-\frac{v e^{2 A_{s}\left(r_{s}\right)}}{2 \pi \ell_{s}^{2}}=-\frac{v e^{2 A\left(r_{s}\right)} \lambda\left(r_{s}\right)^{4 / 3}}{2 \pi \ell_{s}^{2}},
$$

Before proceeding further, we will evaluate the drag force for the conformal case of $\mathcal{N}=4$ SYM where

$$
e^{A_{s}}=\frac{\ell}{r} \quad, \quad v^{2}=f\left(r_{s}\right)=1-\left(\pi T r_{s}\right)^{4} \quad, \quad \frac{\ell^{2}}{\ell_{s}^{2}}=\sqrt{\lambda}
$$

Substituting in (7.17) we obtain, [9]- 11],

$$
F_{\text {conf }}=\frac{\pi}{2} \sqrt{\lambda} T^{2} \frac{v}{\sqrt{1-v^{2}}}
$$

Moving on to YM, to compute the drag force from equation (7.17) we must first determine $\ell_{s}$ in the IHQCD model. In this setup there is no analog of the $\mathcal{N}=4 \mathrm{SYM}$ relation (7.18) between $\ell, \ell_{s}$ and $\lambda$. Rather, the fundamental string length $\ell_{s}$ is determined in a bottom-up fashion, by matching the effective string tension to the QCD string tension $\sigma_{c}$ derived from the lattice calculations. We obtain

$$
\sigma_{c}=\frac{1}{2 \pi \ell_{s}^{2}} e^{2 A_{s, o}\left(r_{*}\right)}=\frac{1}{2 \pi \ell_{s}^{2}} e^{2 A_{o}\left(r_{*}\right)} \lambda_{o}\left(r_{*}\right)^{4 / 3}
$$

where $r_{*}$ is the point where the zero-temperature string scale factor (at $\left.\mathrm{T}=0\right) A_{s, o}(r$ ) has a minimum. For a typical value of $\sigma_{c} \sim(440 \mathrm{MeV})^{2}$ [61] we find

$$
\ell_{s}=6.4 \ell
$$

where $\ell$ is the radius of the asymptotic AdS space.

On the other hand, unlike in $\mathcal{N}=4 \mathrm{SYM}$, in the IHQCD model the value of the coupling $\lambda\left(r_{s}\right)$ in equation (7.17) is not an extra parameter to be fixed by hand, but rather it is determined dynamically together with the background metric. 

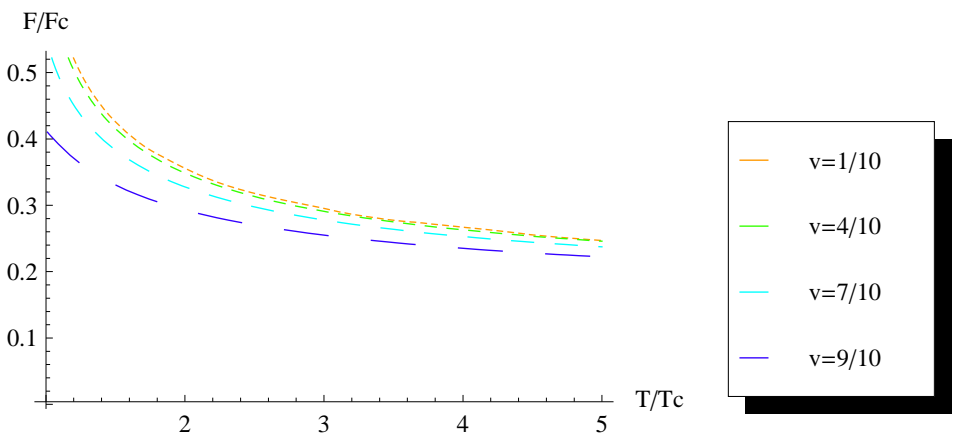

Figure 19: In this figure the ratio of the drag force in improved holographic QCD to the drag force in $\mathcal{N}=4 \mathrm{SYM}$ is shown. The ratio is computed for different velocities as a function of temperature. The 't Hooft coupling for the $\mathcal{N}=4 \mathrm{SYM}$ theory is taken to be 5.5. We chose this value as it is considered in the central region of possible values for the 't Hooft coupling. It is seen that as the velocity increases the value of the ratio decreases.

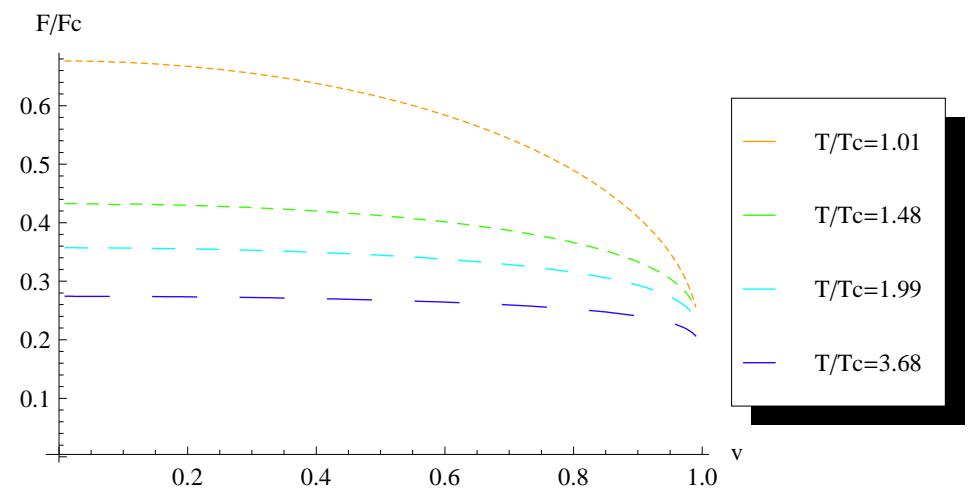

Figure 20: In this figure the ratio of the drag force in improved holographic QCD to the drag force in $\mathcal{N}=4 \mathrm{SYM}$ is shown. The ratio is computed for different temperatures as a function of velocity. The 't Hooft coupling for the $\mathcal{N}=4$ SYM theory is taken to be 5.5. As temperature increases the value of the ratio decreases.

\subsection{The relativistic asymptotics}

When $v \rightarrow 1$ then $r_{s} \rightarrow 0$ and we approach the boundary. Near the boundary $(r \rightarrow 0)$ we have the following asymptotics of the scale factor and the 't Hooft coupling, [14]

$f(r) \simeq 1-\frac{\pi T e^{3 A\left(r_{h}\right)}}{\ell^{3}} r^{4}\left[1+\mathcal{O}\left(\frac{1}{\log (\Lambda r)}\right)\right]+\mathcal{O}\left(r^{8}\right) \quad, \quad e^{A(r)}=\frac{\ell}{r}\left[1+\mathcal{O}\left(\frac{1}{\log (\Lambda r)}\right)\right]+\cdots$

and

$$
\lambda(r)=-\frac{1}{\beta_{0} \log (r \Lambda)}+\mathcal{O}\left(\log (r \Lambda)^{-2}\right)
$$

where $r_{h}$ is the position of the horizon.

We therefore obtain for the turning point

$$
r_{s} \simeq\left[\frac{\ell^{3}\left(1-v^{2}\right)}{\pi T e^{3 A\left(r_{h}\right)}}\right]^{\frac{1}{4}}\left[1+\mathcal{O}\left(\frac{1}{\log \left(1-v^{2}\right)}\right)\right] \quad, \quad \lambda\left(r_{s}\right) \simeq-\frac{4}{\beta_{0} \log \left[1-v^{2}\right]}+\cdots
$$


and the drag force

$$
F_{\text {drag }} \simeq-\frac{\sqrt{\pi T \ell b^{3}\left(r_{h}\right) \lambda^{\frac{8}{3}}\left(r_{s}\right)}}{2 \pi \ell_{s}^{2}} \frac{v}{\sqrt{1-v^{2}}}+\cdots
$$

We also use

$$
e^{3 A\left(r_{h}\right)}=\frac{s(T)}{4 \pi M_{p}^{3} N_{c}^{2}}=\frac{45 \pi \ell^{3} s(T)}{N_{c}^{2}}
$$

where $s(T)$ the entropy per unit three-volume, and we write the relativistic asymptotics of the drag force as,

$$
\begin{gathered}
F_{\text {drag }} \simeq-\frac{\sqrt{\pi T \ell b^{3}\left(r_{h}\right)}}{2 \pi \ell_{s}^{2}} \frac{v}{\sqrt{1-v^{2}}\left(-\frac{\beta_{0}}{4} \log \left[1-v^{2}\right]\right)^{\frac{4}{3}}}+\cdots \\
=-\frac{\ell^{2}}{\ell_{s}^{2}} \sqrt{\frac{45 T s(T)}{4 N_{c}^{2}}} \frac{v}{\sqrt{1-v^{2}}\left(-\frac{\beta_{0}}{4} \log \left[1-v^{2}\right]\right)^{\frac{4}{3}}}+\cdots
\end{gathered}
$$

The force is proportional to the relativistic momentum combination $v / \sqrt{1-v^{2}}$ modulo a power of $\log \left[1-v^{2}\right]$. This factor is present because, as argued in [15] the asymptotic metric is AdS in the Einstein frame instead of the string frame. Its effects are not important phenomenologically.

\subsection{The non-relativistic asymptotics}

We now consider the opposite limit, $v \rightarrow 0$. In this case the turning point asymptotes to the horizon, $r_{s} \rightarrow r_{h}$ and we have the expansion

$$
f(r) \simeq 4 \pi T\left(r_{h}-r\right)+\mathcal{O}\left(\left(r_{h}-r\right)^{2}\right) \quad, \quad r_{s}=r_{h}-\frac{v^{2}}{4 \pi T}+\mathcal{O}\left(v^{4}\right)
$$

and

$$
\begin{gathered}
F_{\text {drag }} \simeq-\frac{e^{2 A\left(r_{h}\right)} \lambda\left(r_{h}\right)^{\frac{4}{3}}}{2 \pi \ell_{s}^{2}} v\left[1-\frac{v^{2}}{2 \pi T} A^{\prime}\left(r_{h}\right)-\frac{v^{2}}{3 \pi T} \frac{\lambda^{\prime}\left(r_{h}\right)}{\lambda\left(r_{h}\right)}+\mathcal{O}\left(v^{4}\right)\right] \\
\simeq-\frac{\ell^{2}}{\ell_{s}^{2}}\left(\frac{45 \pi s(T)}{N_{c}^{2}}\right)^{\frac{2}{3}} \frac{\lambda\left(r_{h}\right)^{\frac{4}{3}}}{2 \pi} v+\mathcal{O}\left(v^{3}\right)
\end{gathered}
$$

where primes are derivatives with respect to the conformal coordinate $r$.

\subsection{The diffusion time}

For a heavy quark with mass $M_{q}$ we may rewrite (7.19) as

$$
F_{\text {conf }} \equiv \frac{d p}{d t}=-\frac{1}{\tau} p \quad, \quad p=\frac{M_{q} v}{\sqrt{1-v^{2}}}
$$

where the first equation defines the diffusion time $\tau$. In the conformal case, the diffusion time is constant,

$$
\tau_{\text {conf }}=\frac{2 M_{q}}{\pi \sqrt{\lambda} T^{2}}
$$


This is not anymore the case in QCD, where $\tau$ defined as above is momentum dependent. We may still define it as in (7.30) in which case we obtain the following limits

$$
\begin{gathered}
\lim _{p \rightarrow \infty} \tau=M_{q} \frac{\ell_{s}^{2}}{\ell^{2}} \sqrt{\frac{4 N_{c}^{2}}{45 T s(T)}}\left(\frac{\beta_{0}}{4} \log \frac{p^{2}}{M_{q}^{2}}\right)^{\frac{4}{3}}+\cdots \\
\lim _{p \rightarrow 0} \tau=M_{q} \frac{\ell_{s}^{2}}{\ell^{2}}\left(\frac{N_{c}^{2}}{45 \pi s(T)}\right)^{\frac{2}{3}} \frac{2 \pi}{\lambda\left(r_{h}\right)^{\frac{4}{3}}}+\cdots
\end{gathered}
$$

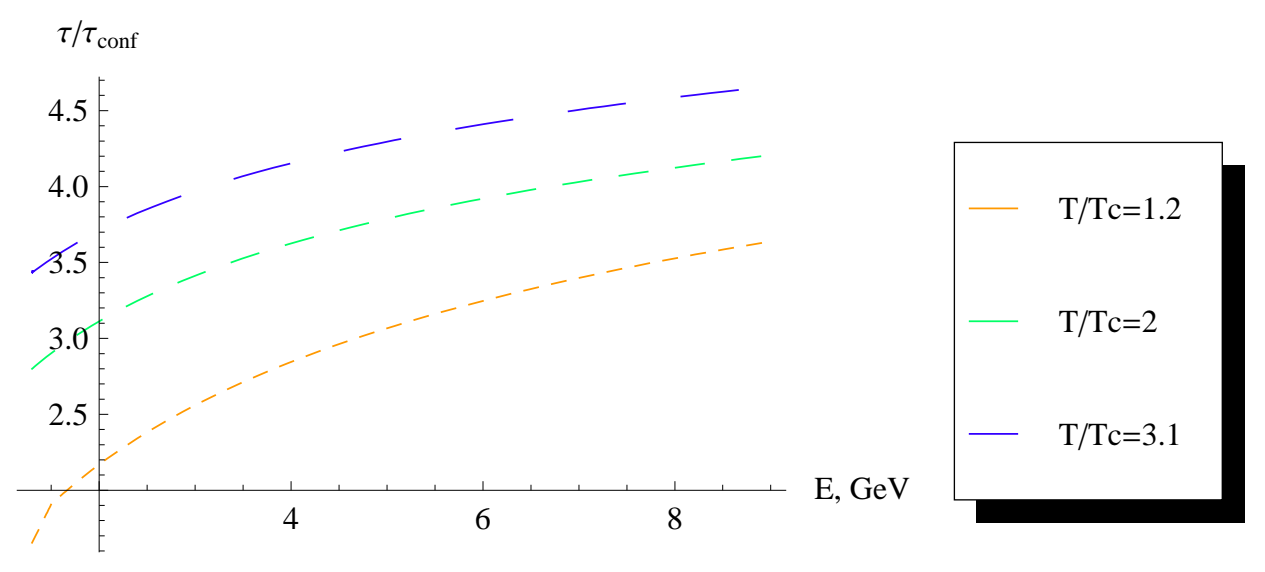

Figure 21: In this figure the ratio of the diffusion time in the Improved Holographic QCD model to the diffusion time in $\mathcal{N}=4 \mathrm{SYM}$ is shown. The 't Hooft coupling for $\mathcal{N}=4 \mathrm{SYM}$ is taken to be $\lambda=5.5$. The heavy quark has a mass of $M_{q}=1.3 \mathrm{GeV}$. Note that with the definition of the diffusion time in (7.30) the ratio is the inverse of the ratio of the forces. A similar plot is valid for the bottom quark as well, as the mass drops out of the ratio. although the energy scales are different. In this plot the $\mathrm{x}$-axis is taken to be in $\mathrm{MeV}$ units. As temperature increases the ratio also increases.

\subsection{Including the medium-induced correction to the quark mass}

In order to estimate the diffusion time of a quark of finite rest mass, we must take into account the fact that the mass of the quark receives medium-induced corrections. In other words, the mass appearing in equation (7.30) is a temperature-dependent quantity, $M_{q}(T) \neq M_{q}(T=0)$. The ratio $M_{q}(T) / M_{q}(0)$ can be estimated holographically by representing a static quark of finite mass by a static, straight string ${ }^{27}$ stretched along the radial direction starting at a point $r=r_{q} \neq 0$. At zero temperature, the IR endpoint of the string can be taken as the "confinement" radius, $r_{*}$, where the string frame metric reaches its minimum value; At finite temperature, the string ends in the IR at the $\mathrm{BH}$ horizon ${ }^{28}$. The

\footnotetext{
${ }^{27}$ This representation ignores the fact that the kinetic mass of a moving quark may be different from the static mass [9].

${ }^{28}$ It would stop at the confinement radius if the latter were closer to the boundary than the horizon, i.e. if $r_{*}(T)<r_{h}(T)$. However, in the model we are considering, in the large BH branch we find that the relation $r_{h}<r_{*}$ is always satisfied.
} 
masses of the quark at zero and finite $T$ are related to the world-sheet action evaluated on the static solution $(\tau=t, \sigma=r)$ :

$$
M_{q}(0)=\frac{\ell}{2 \pi \ell_{s}^{2}} \int_{r_{q}}^{r_{*}} d r e^{2 A_{o}(r)} \lambda_{o}^{4 / 3}(r), \quad M_{q}(T)=\frac{\ell}{2 \pi \ell_{s}^{2}} \int_{r_{q}}^{r_{h}} d r e^{2 A(r)} \lambda^{4 / 3}(r) .
$$

The value $r_{q}$ can be fixed numerically by matching $M_{q}(0)$ to the physical quark mass, and translating the fundamental string tension in physical units by using the relation (7.20), with $\sigma_{c}=(440 M e V)^{2}$. This makes $M_{q}(T)$ a function of $M_{q}(0)$. The ratios $M_{q}(T) / M_{q}(0)$ we found numerically in the model under consideration is shown in figure 22 for the Charm $(M(0)=1.5 \mathrm{GeV})$ and Bottom $(M(0)=4.5 \mathrm{GeV})$ quarks. The fact that, in the deconfined plasma, the quark mass decreases with increasing temperature is a direct consequence of the holographic framework ${ }^{29}$, since for higher temperature, the distance to the horizon is smaller. An indication that this result may be in the right direction comes from the lattice computation of the shift in the position of the quarkonium resonance peak at finite temperature 85]: in the deconfined phase the charmonium peak moves to lower mass at higher temperature. Our result for the medium-induced shift in the constituent quark mass is consistent with these observations.

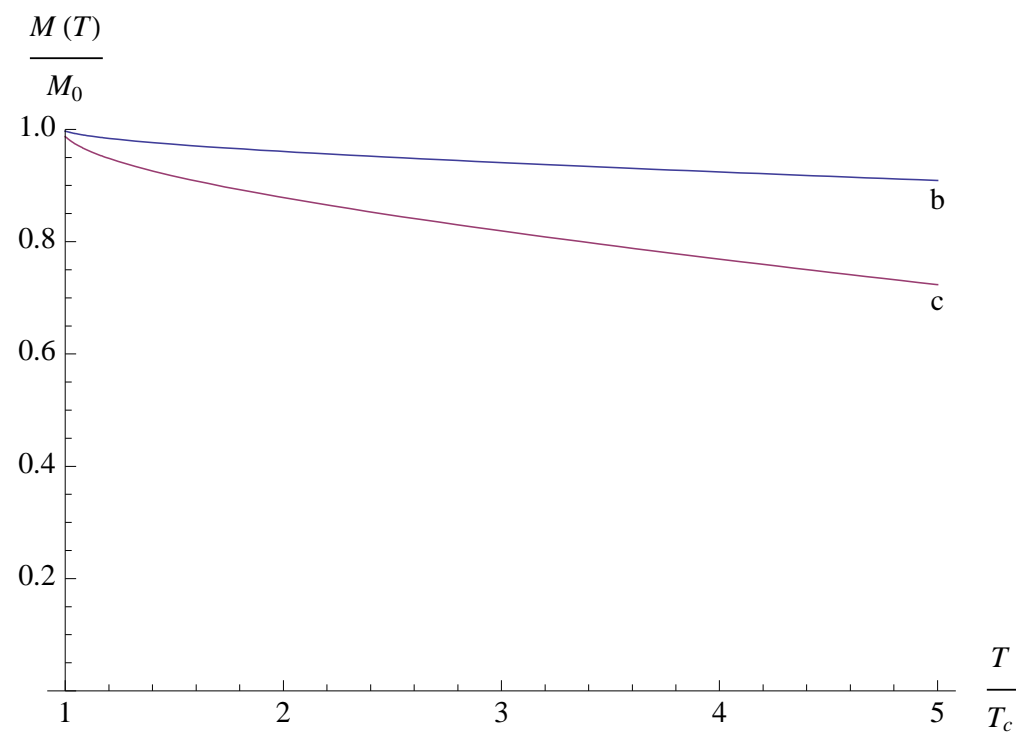

Figure 22: Ratios between the thermal mass and the rest mass of the Charm (curve labeled "c") and Bottom (curve labeled "b" ) quarks, as a function of temperature.

We can now write the diffusion time from eqs. (7.17) and (7.30) as:

$$
\tau(T, v)=\frac{M_{q}(T)}{\sigma_{c} \sqrt{1-v^{2}}}\left(\frac{\lambda_{o}\left(r_{*}\right)}{\lambda\left(r_{s}\right)}\right)^{4 / 3} e^{2 A_{o}\left(r_{*}\right)-2 A\left(r_{s}\right)},
$$

where once again we have eliminated the fundamental string length using equation (7.20). Given a set of zero- and finite-temperature solutions, equation (7.35) can be evaluated

\footnotetext{
${ }^{29}$ For a possible field theoretical explanation of this phenomenon, see 84 .
} 
numerically for different values of the velocity and different quark masses. The results for the Charm $\left(M_{q}(0)=1.5 \mathrm{GeV}\right)$ and Bottom $(M=4.5 \mathrm{GeV})$ quarks are displayed in figure 23.
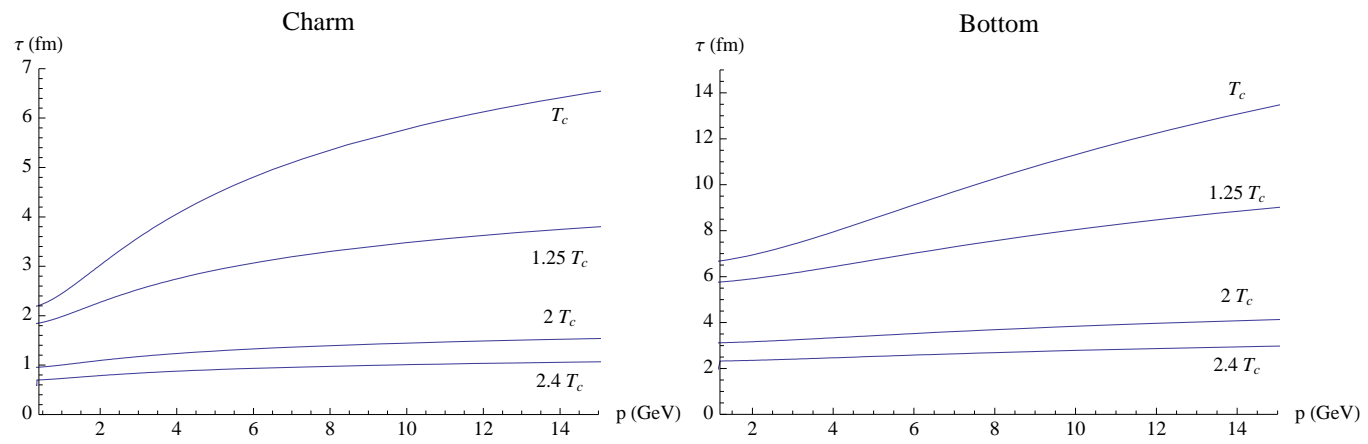

Figure 23: Diffusion time for the Charm and Bottom quarks, as a function of energy, for different ratios of the temperature to the IHQCD transition temperature $T_{c}$.

\subsection{Temperature matching and diffusion time estimates}

An important question is how we should choose the temperature in our holographic model in order to compare our results with heavy-ion collision experiments. This is nontrivial, since our setup is designed to describe pure $S U\left(N_{c}\right)$ Yang-Mills, whereas at RHIC temperatures there are 3 light quark flavors that become relevant. As a consequence, the critical temperatures and the number of degrees of freedom of the two theories are not the same: for pure $S U\left(N_{c}\right)$ Yang Mills we have $N_{c}^{2}-1$ degrees of freedom and a critical temperature around $260 \mathrm{MeV}$; For $S U\left(N_{c}\right)$ QCD with $N_{f}$ flavors the number of degrees of freedom is $N_{c}^{2}-1+N_{c} N_{f}$, and the transition temperature is lower, around $180 \mathrm{MeV}$.

In IHQCD, the transition temperature in physical units was calculated to be $T_{c}=$ $247 \mathrm{MeV}$ [22], i.e. close to the lattice result for the pure YM deconfining temperature. From now on, this is the value we will mean when we refer to $T_{c}$. This is also close to the temperature of QGP at RHIC, which we will denote $T_{Q G P}$, and is estimated to be around $250 \mathrm{MeV}$. Since this value is uncertain, below we give our results for a range of temperatures between $200 \mathrm{MeV}$ and $400 \mathrm{MeV}$. The higher temperatures will be relevant for the LHC heavy-ion collision experiments (see e.g. [86]).

Based on these considerations, there are different ways of fixing the temperature (see e.g. the recent review 38]): one direct and two alternative schemes (that we call the energy and entropy scheme).

- Direct scheme: The temperature of the holographic model is identified with the temperature of the QGP in the experimental situation (at RHIC or LHC), $T_{i h q c d}^{(d i r)}=$ $T_{Q G P}$.

- Energy scheme: One matches the energy densities, rather than the temperatures. The energy density at RHIC is approximately (treating the QCD plasma as a free 
$\operatorname{gas}^{30}$.) $\epsilon_{Q G P} \simeq\left(\pi^{2} / 15\right)\left(N_{c}^{2}-1+N_{c} N_{f}\right)\left(T_{Q G P}\right)^{4}$. For $N_{c}=N_{f}=3$, asking that our energy density matches this value requires us to consider the holographic model at temperature $T_{i h q c d}^{(\epsilon)}$ given by

$$
\epsilon_{i h q c d}\left(T_{i h q c d}^{(\epsilon)}\right) \simeq 11.2\left(T_{Q G P}\right)^{4}
$$

- Entropy scheme: Instead of matching the energy densities, alternatively one can match the entropy density $s$, which for the QGP, in the free gas approximation, is given by $\sigma_{Q G P} \simeq 4 \pi^{2} / 45\left(N_{c}^{2}-1+N_{c} N_{f}\right)\left(T_{Q G P}\right)^{4}$. This leads to the identification:

$$
s_{i h q c d}\left(T_{i h q c d}^{(s)}\right)=14.9\left(T_{Q G P}\right)^{3}
$$

\begin{tabular}{||c|c||c|c||c|c||}
\hline$T_{Q G P}(\mathrm{MeV})$ & $T_{Q G P} / T_{c}$ & $T_{i h q c d}^{(\epsilon)}(\mathrm{MeV})$ & $T_{i h q c d}^{(\epsilon)} / T_{c}$ & $T_{i h q c d}^{(s)}(\mathrm{MeV})$ & $T_{i h q c d}^{(s)} / T_{c}$ \\
\hline 190 & 0.77 & 259 & 1.05 & 274 & 1.11 \\
\hline 220 & 0.89 & 290 & 1.18 & 302 & 1.23 \\
\hline 250 & 1.01 & 325 & 1.31 & 335 & 1.35 \\
\hline 280 & 1.13 & 361 & 1.46 & 368 & 1.49 \\
\hline 310 & 1.26 & 398 & 1.61 & 402 & 1.63 \\
\hline 340 & 1.38 & 434 & 1.76 & 437 & 1.77 \\
\hline 370 & 1.50 & 471 & 1.90 & 472 & 1.91 \\
\hline 400 & 1.62 & 508 & 2.06 & 507 & 2.05 \\
\hline
\end{tabular}

Table 3: Translation table between different temperature identification schemes. The first two columns display temperatures in the direct scheme, (in which the temperature of the holographic model matches the physical QGP temperature) and the corresponding ratio to the IHQCD critical temperature, that was fixed by YM lattice results at $T_{c}=247 \mathrm{MeV}$ [22]. The third and fourth columns display the corresponding temperatures (and respective ratios to $T_{c}$ ) in the energy scheme, and the last two in the entropy scheme.

The temperature translation table between the various schemes is shown in Table 3 . In that table, $T_{c}=247 \mathrm{MeV}$ is the deconfining temperature of the holographic model.

In Figure 24 we show the comparison between the diffusion times, as a function of initial quark momentum, in the different schemes for the Charm and Bottom quarks, at the temperature $T_{Q G P}=250 \mathrm{MeV}$.

The results for the diffusion times at different temperatures, computed at a reference heavy quark initial momentum $p \approx 10 \mathrm{GeV}$, are displayed in Tables 4 and 5 . We see that there is little practical difference between the entropy and energy schemes; on the other hand the difference between the direct scheme and the two alternative schemes can be quite substantial.

\footnotetext{
${ }^{30}$ This is itself an approximation, since as we know both from experiment and in our holographic model, the plasma is strongly coupled up to temperatures of a few $T_{c}$
} 


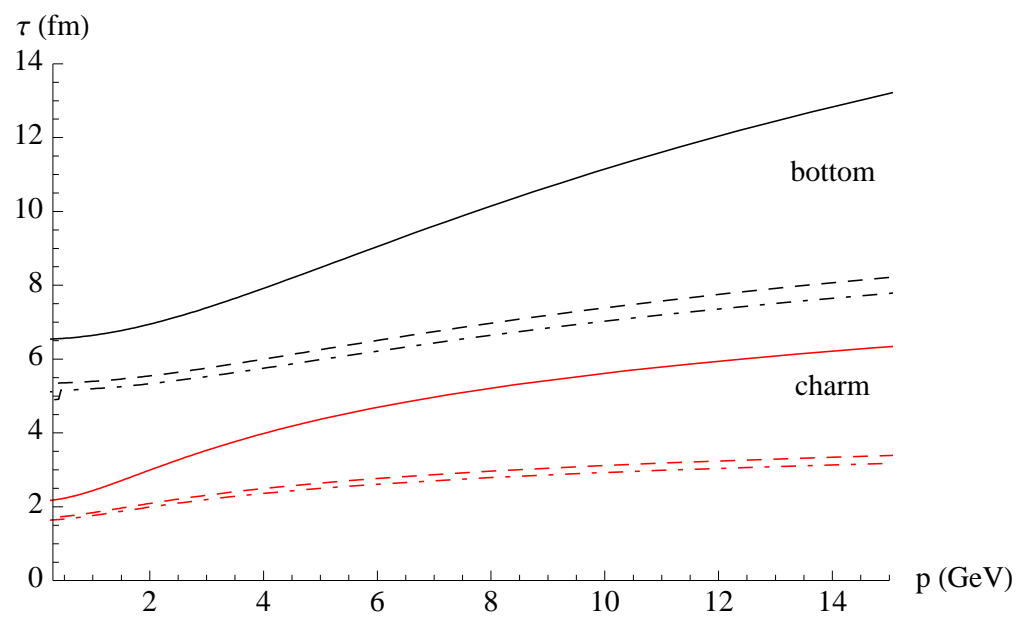

Figure 24: Diffusion times for the Charm and Bottom quarks, as a function of initial momentum, at $T_{Q G P}=250 \mathrm{MeV}$. The different lines represent the in the direct scheme (solid), energy scheme (dashed) and entropy scheme (dash-dotted), all corresponding to the same temperature $T_{Q G P}=250$ $\mathrm{MeV}$.

\begin{tabular}{|c|ccc|}
\hline$T_{Q G P}, M e V$ & $\begin{array}{c}\tau_{\text {charm }}(\mathrm{fm} / \mathrm{c}) \\
\text { (direct) }\end{array}$ & $\begin{array}{c}\tau_{\text {charm }}(\mathrm{fm} / \mathrm{c}) \\
(\text { energy })\end{array}$ & $\begin{array}{c}\tau_{\text {charm }}(\mathrm{fm} / \mathrm{c}) \\
(\text { entropy })\end{array}$ \\
\hline 220 & - & 4.0 & 3.6 \\
\hline 250 & 5.7 & 3.1 & 3.0 \\
\hline 280 & 4.3 & 2.6 & 2.5 \\
\hline 310 & 3.5 & 2.1 & 2.1 \\
\hline 340 & 2.9 & 1.8 & 1.8 \\
\hline 370 & 2.5 & 1.5 & 1.5 \\
\hline 400 & 2.1 & 1.3 & 1.3 \\
\hline
\end{tabular}

Table 4: The diffusion times for the charm quark are shown for different temperatures, in the three different schemes. Diffusion times have been evaluated with a quark initial momentum fixed at $p \approx 10 \mathrm{GeV}$.

\section{Jet quenching parameter}

In this Section we discuss the jet quenching parameter in the class of holographic models under consideration, and we estimate its numerical value for the concrete model with potential (4.1) and parameters fixed as in [22]. For the holographic computation, we will follow [39, 40]. There is another method available [45], but we will not use it here.

The jet-quenching parameter $\hat{q}$ provides a measure of the dissipation of the plasma and it has been associated to the behavior of a Wilson loop joining two light-like lines. We consider two light-like lines which extend for a distance $L^{-}$and are situated distance $L$ apart in a transverse coordinate. Then $\hat{q}$ is given by the large $L^{+}$behavior of the Wilson loop

$$
W \sim e^{-\frac{1}{4 \sqrt{2}} \hat{q} L^{-} L^{2}}
$$




\begin{tabular}{|c|ccc|}
\hline$T_{Q G P}(\mathrm{MeV})$ & $\begin{array}{c}\tau_{\text {bottom }}(\mathrm{fm} / \mathrm{c}) \\
\text { (direct) }\end{array}$ & $\begin{array}{c}\tau_{\text {bottom }}(\mathrm{fm} / \mathrm{c}) \\
(\text { energy })\end{array}$ & $\begin{array}{c}\tau_{\text {bottom }}(\mathrm{fm} / \mathrm{c}) \\
(\text { entropy })\end{array}$ \\
\hline 220 & - & 8.9 & 8.4 \\
\hline 250 & 11.4 & 7.5 & 7.1 \\
\hline 280 & 10.1 & 6.3 & 6.1 \\
\hline 310 & 8.6 & 5.4 & 5.3 \\
\hline 340 & 7.5 & 4.7 & 4.7 \\
\hline 370 & 6.6 & 4.1 & 4.1 \\
\hline 400 & 5.8 & 3.6 & 3.6 \\
\hline
\end{tabular}

Table 5: Diffusion times for the bottom quark are shown for different temperatures, in the three different schemes. Diffusion times have been evaluated with a quark initial momentum fixed at $p \approx 10 \mathrm{GeV}$.

We consider the bulk string frame metric

$$
d s^{2}=e^{2 A_{s}(r)}\left(-f(r) d t^{2}+d \vec{x}^{2}+\frac{d r^{2}}{f(r)}\right) .
$$

To address the problem of the Wilson loop we make a change of coordinates to light cone coordinates for the boundary theory

$$
x^{+}=x_{1}+t \quad x^{-}=x_{1}-t
$$

for which the metric becomes

$$
d s^{2}=e^{2 A_{s}}\left(d x_{2}^{2}+d x_{3}^{2}+\frac{1}{2}(1-f)\left(d x_{+}^{2}+d x_{-}^{2}\right)+(1+f) d x_{+} d x_{-}+\frac{d r^{2}}{f}\right) .
$$

The Wilson loop in question stretches across $x_{2}$, and lies at a constant $x_{+}, x_{3}$. It is convenient to choose a world-sheet gauge in which

$$
x_{-}=\tau, \quad x_{2}=\sigma
$$

Then the action of the string stretching between the two lines is given by

$$
S=\frac{1}{2 \pi \ell_{s}^{2}} \int d \sigma d \tau \sqrt{-\operatorname{det}\left(g_{M N} \partial_{\alpha} X^{M} \partial_{\beta} X^{N}\right)}
$$

and assuming a profile of $r=r(\sigma)$ we obtain

$$
S=\frac{L^{-}}{2 \pi \ell_{s}^{2}} \int d x_{2} e^{2 A_{s}} \sqrt{\frac{(1-f)}{2}\left(1+\frac{r^{\prime 2}}{f}\right)} .
$$

The integrand does not depend explicitly on $x_{2}$, so there is a conserved quantity, $c$ :

$$
r^{\prime} \frac{\partial \mathcal{L}}{\partial r^{\prime}}-\mathcal{L}=\frac{c}{\sqrt{2}}
$$




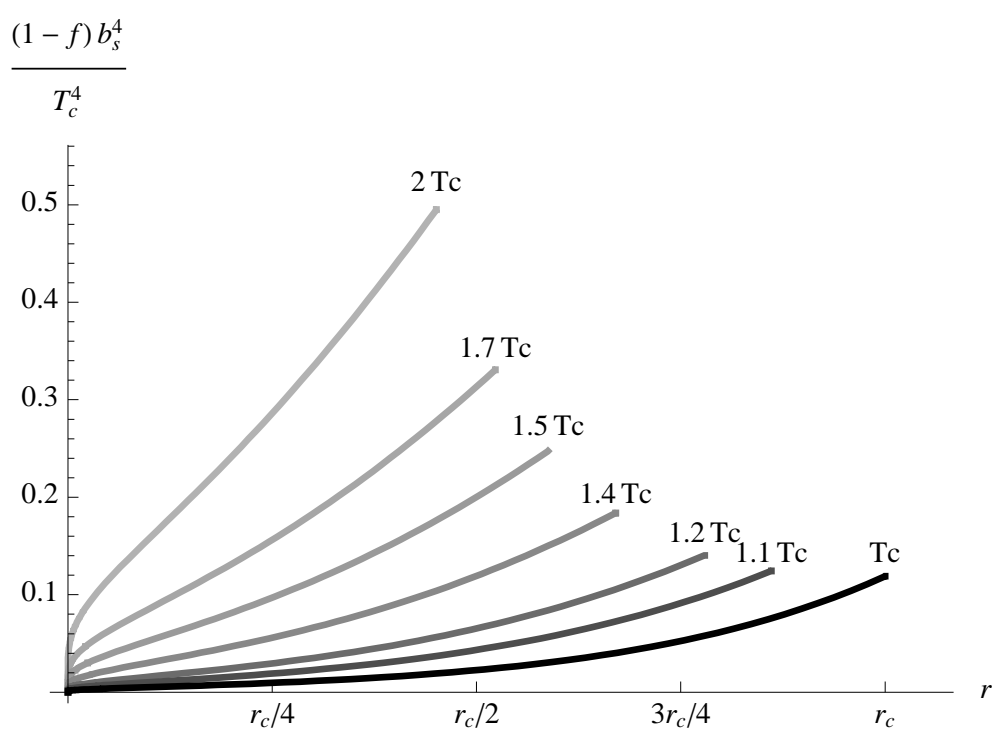

Figure 25: In this figure the combination $(1-f) e^{4 A_{s}}$ is plotted as a function of the radial distance, for several temperatures. The radial distance is given in units of the horizon position $r_{c}$ for the black hole at the critical temperature $T_{c}$. All curves stop at the corresponding horizon position.

which leads to

$$
r^{\prime 2}=f\left(\frac{e^{4 A_{s}}(1-f)}{c^{2}}-1\right) .
$$

A first assessment of this relation involves determining the zeros and the region of positivity of the right-hand side. $f$ is always positive and vanishes at the horizon. For the second factor we need the asymptotics of $e^{4 A_{s}}(1-f)$. This factor remains positive and bounded from below in the interior and up to the horizon. It vanishes however logarithmically near the boundary as

$$
e^{4 A_{s}}(1-f)=\pi T \ell e^{3 A\left(r_{h}\right)}\left(-\frac{1}{\beta_{0} \log (\Lambda r)}\right)^{\frac{8}{3}}\left[1+\mathcal{O}\left(\frac{1}{\log (\Lambda r)}\right)\right]
$$

This is unlike the conformal case where we obtain a constant

$$
\left.e^{4 A_{s}}(1-f)\right|_{\text {conformal }}=(\pi T \ell)^{4}
$$

Because of this, for fixed $c$, there is a region near the boundary where $r^{2}$ becomes negative. At this stage we will avoid this region, by using a modified boundary at $r=\epsilon$. We will later show that this gymnastics will be irrelevant for the computation of the jet quenching parameter, as it involves effectively the limit $c \rightarrow 0$.

We will place the modified boundary $r=\epsilon$ a bit inward from the place $r=r_{\text {min }}$ where the factor $\frac{e^{4 A_{s}}(1-f)}{c^{2}}-1$ vanishes:

$$
e^{4 A_{s}\left(r_{\text {min }}\right)}\left(1-f\left(r_{\text {min }}\right)\right)=c^{2}
$$

Therefore we choose $r_{\min }<\epsilon$. 
Then, in the range $\epsilon<r<r_{h}$ the factor $\frac{e^{4 A_{s}}(1-f)}{c^{2}}-1$ is positive for sufficiently small $c$. In this same range, $r^{\prime}$ vanishes only at $r=r_{h}$. This is the true turning point of the string world-sheet. This is also what happens in the conformal case. It is also intuitively obvious that the relevant Wilson loop must sample also the region near the horizon.

The constant $c$ is determined by the fact that the two light-like Wilson loops are a $x_{2}=L$ distance apart.

$$
\frac{L}{2}=\int_{\epsilon}^{r_{h}} \frac{c d r}{\sqrt{f\left(e^{4 A_{s}}(1-f)-c^{2}\right)}} .
$$

The denominator vanishes at the turning point. The singularity is integrable ${ }^{31}$. Therefore, as we are interested in the small $L$ region, it is obvious from the expression above that that $c$ must also be small in the same limit.

This relation can then be expanded in powers of $c$ as

$$
\frac{L}{2 c}=\int_{\epsilon}^{r_{h}} \frac{e^{-2 A_{s}} d r}{\sqrt{f(1-f)}}+\frac{c^{2}}{2} \int_{\epsilon}^{r_{h}} \frac{e^{-6 A_{s}} d r}{\sqrt{f(1-f)^{3}}}+\mathcal{O}\left(c^{4}\right) .
$$

Therefore to leading order in $L$

$$
c=\frac{L}{2 \int_{\epsilon}^{r_{h}} \frac{e^{-2 A_{s} d r}}{\sqrt{f(1-f)}}}+\mathcal{O}\left(L^{3}\right)
$$

We are now ready to evaluate the Nambu-Goto action of the extremal configuration we have found. Starting from (8.7), we substitute $r^{\prime}$ from (8.9), and change integration variable from $x_{2} \rightarrow r$ to obtain

$$
S=\frac{2 L^{-}}{2 \pi \ell_{s}^{2}} \int_{\epsilon}^{r_{h}} d r \frac{e^{4 A_{s}}(1-f)}{\sqrt{2 f\left(e^{4 A_{s}}(1-f)-c^{2}\right)}} .
$$

As in [39, 40], we subtract from equation (8.16) the action of two free string straight world-sheets that hang down to the horizon. To compute this action a convenient choice of gauge is $x_{-}=\tau, \quad r=\sigma$. The action of each sheet is

$$
S_{0}=\frac{L^{-}}{2 \pi \ell_{s}^{2}} \int_{\epsilon}^{r_{h}} d r \sqrt{g_{--} g_{r r}}=\frac{L^{-}}{2 \pi \ell_{s}^{2}} \int_{\epsilon}^{r_{h}} d r e^{2 A_{s}} \sqrt{\frac{1-f}{2 f}}
$$

The subtracted action is therefore:

$$
S_{r}=S-2 S_{0}=\frac{L^{-} c^{2}}{2 \pi \ell_{s}^{2}} \int_{\epsilon}^{r_{h}} \frac{d r}{e^{2 A_{s}} \sqrt{f(1-f)}}+\mathcal{O}\left(c^{4}\right),
$$

Using now (8.15) to substitute $c$ we finally obtain

$$
S_{r}=\frac{L^{-} L^{2}}{8 \pi \ell_{s}^{2}} \frac{1}{\int_{\epsilon}^{r_{h}} \frac{d r}{e^{2 A_{s}} \sqrt{f(1-f)}}}+\mathcal{O}\left(L^{4}\right)
$$

\footnotetext{
${ }^{31}$ Even if we choose $\epsilon=r_{\text {min }}$, the new singularity at $r=r_{\min }$ is also integrable as suggested from (8.10).
} 
So far we have evaluated the relevant Wilson loop in the fundamental representation (by using probe quarks). On the other hand, the Wilson loop that defines the jet-quenching parameter is an adjoint one. We can obtain it in the large- $N_{c}$ limit from the fundamental using $t r_{\text {Adjoint }}=t r_{\text {Fundamental }}^{2}$. We finally extract the jet-quenching parameter as

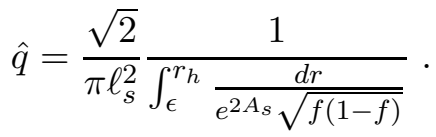

We are now ready to remove the cutoff. As the integral appearing is now well-defined up to the real boundary $r=0$ we may rewrite it as

$$
\int_{\epsilon}^{r_{h}} \frac{e^{-2 A_{s}} d r}{\sqrt{f(1-f)}}=\int_{0}^{r_{h}} \frac{e^{-2 A_{s}} d r}{\sqrt{f(1-f)}}-I(\epsilon) \quad, \quad I(\epsilon)=\int_{0}^{\epsilon} \frac{e^{-2 A_{s}} d r}{\sqrt{f(1-f)}}
$$

In [76] we obtain the small $\epsilon$ estimate of $I(\epsilon)$ that vanishes as $\sim \epsilon(\log \epsilon)^{\frac{4}{3}}$. We may finally write ${ }^{32}$

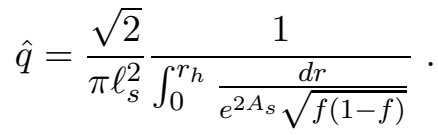

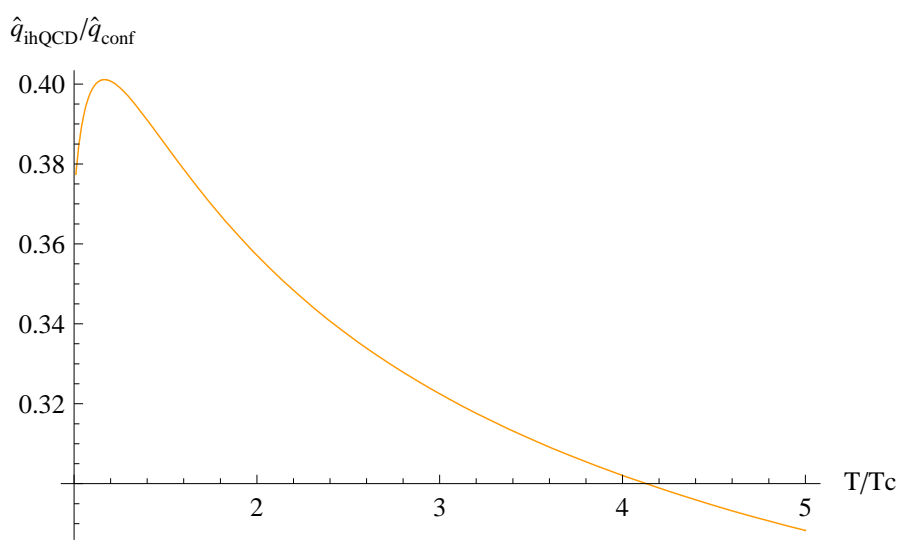

Figure 26: In this figure the ratio of the jet quenching parameter in our model to the jet quenching parameter in $\mathcal{N}=4$ is shown. The integral present in equation 8.20 ) has been numerically calculated from an effective cutoff at $r=r_{h} / 1000$. The jet quenching parameter in $\mathcal{N}=4 \mathrm{SYM}$ has been calculated with $\lambda_{\prime \prime H o o f t}=5.5$.

From equation (8.22) we obtain, in the conformal case:

$$
\hat{q}_{\text {conformal }}=\frac{\Gamma\left[\frac{3}{4}\right]}{\Gamma\left[\frac{5}{4}\right]} \sqrt{2 \lambda} \pi^{\frac{3}{2}} T^{3}
$$

The conformal value, for the median value of $\lambda=5.5$ and $T \simeq 250 \mathrm{MeV}$ gives $\hat{q}_{\text {conformal }} \simeq$ $1.95 \mathrm{GeV}^{2} / \mathrm{fm}$ where we used the conversion $1 \mathrm{GeV} \simeq 5 \mathrm{fm}^{-1}$.

\footnotetext{
${ }^{32}$ In practise, the previous discussion including regularizing the UV is academic. The numerical calculation is done with a finite cutoff where the boundary conditions for the couplings are imposed.
} 


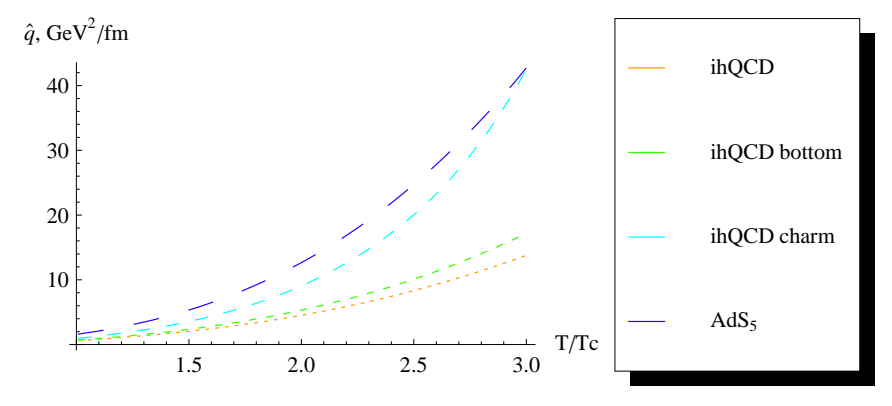

Figure 27: The jet quenching parameter $\hat{q}$ for the Improved Holographic QCD model and $\mathcal{N}=4$ SYM is shown in units of $\mathrm{GeV}^{2} / \mathrm{fm}$ for a region close to $T=T_{c}$. The smallest dashed curve is the ihQCD result with an effective cutoff of $r_{\text {cutof } f}=r_{h} / 1000$. The small dashed curve is the ihQCD result with the cutoff from the mass of the Bottom quark. The medium dashed curve has a cutoff coming from the Charm mass and and largest dashed curve is the $\mathcal{N}=4 \mathrm{SYM}$ result.

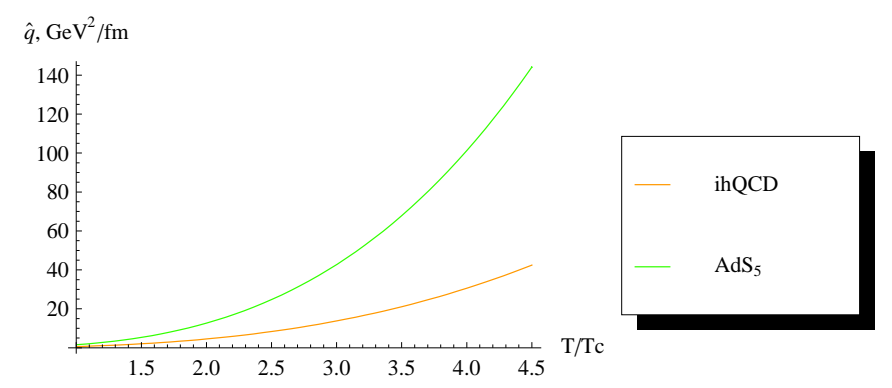

Figure 28: The jet quenching parameter $\hat{q}$ for the Improved Holographic QCD model (lower curve) and $\mathcal{N}=4 \mathrm{SYM}$ (upper curve) are shown in units of $\mathrm{GeV}^{2} / \mathrm{fm}$ for temperatures up to $T=4 T_{c}$.

\begin{tabular}{|c|cc|}
\hline$T_{Q G P}, \mathrm{MeV}$ & $\begin{array}{c}\hat{q}\left(\mathrm{GeV}^{2} / \mathrm{fm}\right) \\
\text { (direct) }\end{array}$ & $\begin{array}{c}\hat{q}_{1}\left(\mathrm{GeV}^{2} / \mathrm{fm}\right) \\
\text { (direct) }\end{array}$ \\
\hline 220 & - & - \\
\hline 250 & 0.5 & 0.6 \\
\hline 280 & 0.8 & 0.8 \\
\hline 310 & 1.1 & 1.1 \\
\hline 340 & 1.4 & 1.4 \\
\hline 370 & 1.8 & 1.8 \\
\hline 400 & 2.2 & 2.2 \\
\hline
\end{tabular}

Table 6: This table shows the jet quenching parameter $\hat{q}$ computed with different cutoffs for the different temperatures shown in the first column. The computation is done in the direct scheme. The second column shows $\hat{q}$ with a cutoff at $r_{\text {cutoff }}=r_{h} / 1000$, where $r_{h}$ is the location of the horizon. In accordance with the conclusions of appendix $\hat{q}$ does not change significantly as we vary the cutoff from $r_{h} / 1000$ to $r_{h} / 100$.

Numerical evaluation of equation (8.22) in the non-conformal IHQCD setup ${ }^{33}$ gives us a value of $\hat{q}$ which is lower (at a given temperature) than the conformal value, as

\footnotetext{
${ }^{33}$ In this case, the value of $\ell_{s}$ appearing in equation 8.22 is fixed as explained in Section 4.
} 


\begin{tabular}{|c|c|c|c|}
\hline$T_{Q G P}, M e V$ & $\begin{array}{c}\hat{q}\left(G e V^{2} / f m\right) \\
\text { (direct) }\end{array}$ & $\begin{array}{c}\hat{q}\left(G e V^{2} / f m\right) \\
(\text { energy })\end{array}$ & $\begin{array}{c}\hat{q}\left(\mathrm{GeV}^{2} / \mathrm{fm}\right) \\
\text { (entropy) }\end{array}$ \\
\hline 220 & - & 0.9 & 1.0 \\
\hline 250 & 0.5 & 1.2 & 1.3 \\
\hline 280 & 0.8 & 1.6 & 1.7 \\
\hline 310 & 1.1 & 2.1 & 2.2 \\
\hline 340 & 1.4 & 2.7 & 2.8 \\
\hline 370 & 1.8 & 3.4 & 3.4 \\
\hline 400 & 2.2 & 4.2 & 4.2 \\
\hline
\end{tabular}

Table 7: This table displays the jet quenching parameter $\hat{q}$ using the three different comparison schemes. For lower temperatures the "entropy scheme" gives higher values. As energy is increased the energy and entropy schemes temperatures start to coincide and there is little difference in the jet quenching parameter as well.

\begin{tabular}{|c|ccc|}
\hline$T_{Q G P}, \mathrm{MeV}$ & $\begin{array}{c}\hat{q}_{\text {charm }}\left(\mathrm{GeV}^{2} / \mathrm{fm}\right) \\
\text { (direct) }\end{array}$ & $\begin{array}{c}\hat{q}_{\text {charm }}\left(\mathrm{GeV}^{2} / \mathrm{fm}\right) \\
\text { (energy) }\end{array}$ & $\begin{array}{c}\hat{q}_{\text {charm }}\left(\mathrm{GeV}^{2} / \mathrm{fm}\right) \\
\text { (entropy) }\end{array}$ \\
\hline 220 & - & 1.3 & 1.5 \\
\hline 250 & 0.8 & 1.8 & 2.0 \\
\hline 280 & 1.2 & 2.6 & 2.8 \\
\hline 310 & 1.7 & 3.5 & 3.6 \\
\hline 340 & 2.2 & 4.6 & 4.7 \\
\hline 370 & 2.8 & 5.9 & 6.0 \\
\hline 400 & 3.6 & 7.6 & 7.5 \\
\hline
\end{tabular}

Table 8: This table displays the jet quenching parameter $\hat{q}$ using the three different comparison schemes with an effective cutoff provided by the mass of the Charm quark. Again, for lower temperatures the "entropy scheme" gives higher values. As energy is increased the energy and entropy schemes temperatures start to coincide and there is little difference in the jet quenching parameter as well. Also when the temperature approaches the quark mass the picture of the heavy quark as a hanging string collapses and results are not reliable.

shown in Figures 26, 27 and 28. Tables 6 to 9 display the numerical values of the jet quenching parameter at different temperatures in the experimentally relevant range, in different temperature matching schemes.

\section{Discussion and outlook}

The construction presented in this paper offers a holographic description of large- $N_{c}$ YangMills theory that is both realistic and calculable, and in quite good agreement with a large number of lattice results both at zero and finite temperature.

It is a phenomenological model; as such it is not directly associated to an explicit string theory construction. In this respect it is in the same class as the models based on pure AdS backgrounds (with hard or soft walls) [7, 8, 87, 88], or on IR deformations of the AdS metric [89, 90]. In comparison to them however, the IHQCD approach has the advantage 


\begin{tabular}{|c|ccc|}
\hline$T_{Q G P}, \mathrm{MeV}$ & $\begin{array}{c}\hat{q}_{\text {bottom }}\left(\mathrm{GeV}^{2} / \mathrm{fm}\right) \\
\text { (direct) }\end{array}$ & $\begin{array}{c}\hat{q}_{\text {bottom }}\left(\mathrm{GeV}^{2} / \mathrm{fm}\right) \\
\text { (energy) }\end{array}$ & $\begin{array}{c}\hat{q}_{\text {bottom }}\left(\mathrm{GeV}^{2} / \mathrm{fm}\right) \\
\text { (entropy) }\end{array}$ \\
\hline 220 & - & 1.0 & 1.1 \\
\hline 250 & 0.6 & 1.4 & 1.5 \\
\hline 280 & 0.9 & 1.9 & 2.0 \\
\hline 310 & 1.2 & 2.5 & 2.6 \\
\hline 340 & 1.6 & 3.2 & 3.2 \\
\hline 370 & 2.0 & 4.0 & 4.0 \\
\hline 400 & 2.5 & 5.0 & 4.9 \\
\hline
\end{tabular}

Table 9: This table displays the jet quenching parameter $\hat{q}$ using the three different comparison schemes with an effective cutoff provided by the mass of the Bottom quark. The results are close to the $\hat{q}$ results computed in Table 7 since the mass of the Bottom quark is much larger than the temperatures we examine.

that the dynamics responsible for strong coupling phenomena (such as confinement and phase transitions) is made explicit in the bulk description, and it is tied to the fact that the coupling constant depends on the energy scale and becomes large in the IR. This makes the model consistent and calculable, once the 5D effective action is specified: the dynamics can be entirely derived from the bulk Einstein's equation. The emergence of an IR mass scale and the finite temperature phase structure are built-in: they need not be imposed by hand and do not suffer from ambiguities related to IR boundary conditions (as in hard wall models) or from inconsistencies in the laws of thermodynamics (as in non-dynamical soft wall models based on a fixed dilaton profile [8] or on a fixed metric [89, 90]). More specifically, in this approach it is guaranteed that the Bekenstein-Hawking temperature of the black hole matches the entropy computed as the derivative of the free energy with respect to temperature.

With an appropriate choice of the potential, a realistic and quantitatively accurate description of essentially all the static properties (spectrum and equilibrium thermodynamics) of the dynamics of pure Yang-Mills can be provided. The main ingredient responsible for the dynamics (the dilaton potential) is fixed through comparison with both perturbative QCD and lattice results. It is worth stressing that such a matching on the quantitative level was only possible because the class of holographic models we discuss generically provides a qualitatively accurate description of the strong Yang-Mills dynamics. This is a highly non-trivial fact, that strongly indicates that a realistic holographic description of real-world QCD might be ultimately possible.

Although the asymptotics of our potential is dictated by general principles, we base our choice of parameters by comparing with the lattice results for the thermodynamics. There are other physical parameters in the $5 \mathrm{D}$ description that do not appear in the potential: the 5D Planck scale, that was fixed by matching the free field thermodynamics in the limit $T \rightarrow \infty$; the coefficients in the axion kinetic term, that were set by matching the axial glueball spectrum and the topological susceptibility (from the lattice). The quantities that we use as input in our fit, as well as the corresponding parameters in the 5D model, are 
shown in the upper half of Table 2 .

The fact that our potential has effectively two free parameters depends on our choice of the functional form. This functional form contains some degree of arbitrariness, in that only the UV and IR asymptotics of $V(\lambda)$ are fixed by general considerations (matching the perturbative $\beta$-function in the UV, and a discrete linear glueball spectrum for the IR). Therefore the results presented in this paper offer more a description, rather than a prediction of the thermodynamics.

Nevertheless, there are several quantities that we successfully "postdict" (i.e. they agree with the lattice results) once the potential is fixed: apart from the good agreement of the thermodynamic functions over the whole range of temperature explored by the lattice studies (see Figures 5, 7 and 9), they are the lowest glueball mass ratios and the value of the critical temperature. The comparison of these quantities with the lattice results is shown in the lower half of Table 2, and one can see that the agreement is overall very good. Moreover the model predicts the masses of the full towers of glueball states in the $0^{+-}, 0^{++}, 2^{++}$families.

The fact that IHQCD is consistent with a large number of lattice results is clearly not the end of the story: its added value, and one of the main reasons for its interest lies in its immediate applicability beyond equilibrium thermodynamics, i.e. in the dynamic regimes tested in heavy-ion collision experiments. This is a generic feature of the holographic approach, in which there are no obstructions (as opposed to the lattice) to perform realtime computations and to calculate hydrodynamics and transport coefficients. IHQCD provides a framework to compute these quantities in a case where the static properties agree with the real-word QCD at the quantitative level. Therefore the bulk viscosity, drag force and jet quenching parameters were computed in IHQCD:

Bulk Viscosity: The bulk viscosity was computed by calculating the low frequency asymptotics of the appropriate stress tensor correlator holographically. The result is that the bulk viscosity rises near the phase transition but stays always below the shear viscosity. It floats somewhat above the Buchel bound, with a coefficient of proportionality varying between 1 and 2 . Therefore it is expected to affect the elliptic flow at the small percentage level [80, 33]. Knowledge of the bulk viscosity is important in extracting the shear viscosity from the data. This result is not in agreement with the lattice result near $T_{c}$. In particular the lattice result gives a value for the viscosity that is ten times larger.

The bulk viscosity keeps increasing in the black-hole branch below the transition point until the large $\mathrm{BH}$ turns into the small $\mathrm{BH}$ at a temperature $T_{\min }$. The bulk viscosity on the small $\mathrm{BH}$ background is always larger than the respective one in the large $\mathrm{BH}$ background. In particular, it can be shown that the $\mathrm{T}$ derivative of the quantity $\zeta / s$ diverges at $T_{\text {min }}$. This is the holographic reason for the presence of a peak in $\zeta / s$ near $T_{c}$. On the other hand, as it is shown in [19], the presence of $T_{\min }$ (i.e. a small $\mathrm{BH}$ branch) is in one-to-one correspondence with color confinement at zero $\mathrm{T}$. We arrive thus at the suggestion that in a (large N) gauge theory that confines color at zero $\mathrm{T}$, there shall be a rise in $\zeta / s$ near $T_{c}$.

An important ingredient here is the value of the viscosity asymptotically in the small $\mathrm{BH}$ branch. There the asymptotic value correlates to the IR behavior of the potential. 
Taking also into account the fact that this asymptotic value is very close to the value of the bulk viscosity near $T_{c}$, we can derive bounds that suggest that the bulk viscosity cannot increase a lot near $T_{c}$.

Drag Force: The drag force calculated from IHQCD has the expected behavior. Although it increases with temperature, it does so slower than in $\mathcal{N}=4 \mathrm{SYM}$, signaling the effects of asymptotic freedom.

Diffusion Time: Based on the drag force calculation the diffusion times can be computed for a heavy external quark. The numerical values obtained are in agreement with phenomenological models 37. To accommodate for the fact that IHQCD exhibits a phase transition around $T=247 \mathrm{MeV}$ (i.e. about 30\% higher than in QCD), the results are compared using alternative schemes, as proposed in [11. For example, for an external Charm quark of momentum $p=10 \mathrm{GeV}$ (in the alternative scheme) a diffusion time of $\tau=2.6 \mathrm{fm}$ at temperature $T=280 \mathrm{MeV}$ is found. Similarly, for a Bottom quark of the same momentum and at the same temperature, $\tau=6.3 \mathrm{fm}$. Generally the numbers obtained are close to those obtained by [37] and 442].

Jet Quenching: The jet quenching parameter of this model, has been also calculated, based on the formalism of [39, 40] by computing the appropriate light-like Wilson loop. $\hat{q}$ grows with temperature, but slower than the $T^{3}$ growth of $\mathcal{N}=4 \mathrm{SYM}$ result. Again this can be attributed to the incorporation of asymptotic freedom in IHQCD. Using the alternative scheme to compare with experiment, the results are close to the lower quoted values of $\hat{q}$. For example, for a temperature of $T=290 \mathrm{MeV}$, which in the alternative "energy scheme" corresponds to a temperature of $T=395 \mathrm{MeV}$ in our model, we find that $\hat{q} \approx 2 G e V^{2} / f m$.

However, the numbers obtained for this particular definition of jet quenching parameter seem rather low and indicate that this may not be the most appropriate definition in the holographic context. There are other ways to define $\hat{q}$, in particular using the fluctuations of the trailing string solution. This is gives a direct and more detailed input in the associated Langevin dynamics and captures the asymmetry between longitudinal and transverse fluctuations. This can be computed along the lines set in 445, 47, 48] and the results were recently reported in [91]. 


\section{Acknowledgments}

We would like to thank the numerous colleagues that have shared their insights with us via discussions and correspondence since 2007 that this line of research has started: O. Aharony, L. Alvarez-Gaumé, B. Bringoltz, R. Brower, M. Cacciari, J. Casalderrey-Solana, R. Emparan , F. Ferrari, B. Fiol, P. de Forcrand, R. Granier de Cassagnac, L. Giusti, S. Gubser, K. Hashimoto, T. Hertog, U. Heinz, D. K. Hong, G. Horowitz, E. Iancu, K. Intriligator, K. Kajantie, F. Karsch, D. Kharzeev, D. Kutasov, H. Liu, B. Lucini, M. Luscher, J. Mas, D. Mateos, H.B. Meyer, C. Morningstar, V. Niarchos, C. Nunez, Y. Oz, H. Panagopoulos, S. Pal, M. Panero, I. Papadimitriou, A. Paredes, A. Parnachev, G. Policastro, S. Pufu, K. Rajagopal, F.Rocha, P. Romatchke, C. Salgado, F. Sannino, M. Shifman, E. Shuryak, S. J. Sin, C. Skenderis, D. T. Son, J. Sonnenschein, S. Sugimoto, M. Taylor, M. Teper, J. Troost, A. Tseytlin, A. Vainshtein, G. Veneziano, A. Vladikas, L. Yaffe, A. Yarom and U. Wiedemann.

This work was partially supported by a European Union grant FP7-REGPOT-20081-CreteHEPCosmo-228644, and ANR grant NT05-1-41861. Work of LB has been partly

funded by INFN, Ecole Polytechnique (UMR du CNRS 7644), MEC and FEDER under grant FPA2008-01838, by the Spanish Consolider-Ingenio 2010 Programme CPAN (CSD2007-00042) and by Xunta de Galicia (Consellería de Educación and grant PGIDIT06PXIB206185PR).

E. K. thanks the organizers and especially E. Papantonopoulos for organizing a very interesting and stimulating school. 


\section{References}

[1] J. Adams et al. [STAR Collaboration], "Experimental and theoretical challenges in the search for the quark gluon plasma: The STAR collaboration's critical assessment of the evidence from RHIC collisions," Nucl. Phys. A 757 (2005) 102 [ArXiv:nucl-ex/0501009];

B. B. Back et al., "The PHOBOS perspective on discoveries at RHIC," Nucl. Phys. A $\mathbf{7 5 7}$ (2005) 28 [ArXiv:nucl-ex/0410022];

I. Arsene et al. [BRAHMS Collaboration], "Quark gluon plasma and color glass condensate at RHIC? The perspective from the BRAHMS experiment," Nucl. Phys. A 757 (2005) 1 [ArXiv:nucl-ex/0410020],

K. Adcox et al. [PHENIX Collaboration], "Formation of dense partonic matter in relativistic nucleus nucleus collisions at RHIC: Experimental evaluation by the PHENIX collaboration," Nucl. Phys. A 757 (2005) 184 [ArXiv:nucl-ex/0410003].

[2] M. Luzum and P. Romatschke, "Conformal Relativistic Viscous Hydrodynamics: Applications to RHIC results at $\sqrt{s_{N N}}=200 \mathrm{GeV}$," Phys. Rev. C 78 (2008) 034915 [ArXiv:0804.4015][nucl-th].

[3] G. Policastro, D. T. Son and A. O. Starinets, "The shear viscosity of strongly coupled $N=4$ supersymmetric Yang-Mills plasma," Phys. Rev. Lett. 87 (2001) 081601

[ArXiv:hep-th/0104066];

P. Kovtun, D. T. Son and A. O. Starinets, "Viscosity in strongly interacting quantum field theories from black-hole physics," Phys. Rev. Lett. 94 (2005) 111601 [arXiv:hep-th/0405231].

[4] E. Shuryak, "Physics of Strongly coupled Quark-Gluon Plasma," [ArXiv:0807.3033][hep-ph]; D. T. Son and A. O. Starinets, "Viscosity, Black Holes, and Quantum Field Theory," Ann. Rev. Nucl. Part. Sci. 57 (2007) 95 [ArXiv:0704.0240][hep-th]];

M. Natsuume, "String theory and quark-gluon plasma," [ArXiv:hep-ph/0701201].

[5] F. Karsch, D. Kharzeev and K. Tuchin, "Universal properties of bulk viscosity near the QCD phase transition," Phys. Lett. B 663 (2008) 217 [ArXiv:0711.0914][hep-ph].

[6] H. B. Meyer, "A calculation of the bulk viscosity in SU(3) gluodynamics," Phys. Rev. Lett. 100 (2008) 162001 [ArXiv:0710.3717][hep-lat]; "Energy-momentum tensor correlators and viscosity," [ArXiv:0809.5202][hep-lat].

[7] J. Erlich, E. Katz, D. T. Son and M. A. Stephanov, "QCD and a holographic model of hadrons," Phys. Rev. Lett. 95, 261602 (2005) [arXiv:hep-ph/0501128];

L. Da Rold and A. Pomarol, "Chiral symmetry breaking from five dimensional spaces," Nucl. Phys. B 721, 79 (2005) [ArXiv:hep-ph/0501218.

[8] A. Karch, E. Katz, D. T. Son and M. A. Stephanov, "Linear confinement and AdS/QCD," Phys. Rev. D 74 (2006) 015005 [ArXiv:hep-ph/0602229].

[9] C. P. Herzog, A. Karch, P. Kovtun, C. Kozcaz and L. G. Yaffe, "Energy loss of a heavy quark moving through $N=4$ supersymmetric Yang-Mills plasma," JHEP 0607 (2006) 013; [ArXiv:hep-th/0605158.

[10] H. Liu, K. Rajagopal and U. A. Wiedemann, "Calculating the jet quenching parameter from AdS/CFT," Phys. Rev. Lett. 97 (2006) 182301 [ArXiv:hep-ph/0605178].

[11] S. S. Gubser, "Drag force in AdS/CFT," Phys. Rev. D 74 (2006) 126005 [ArXiv:hep-th/0605182];

"Comparing the drag force on heavy quarks in $N=4$ super-Yang-Mills theory and QCD," Phys. Rev. D 76 (2007) 126003 [ArXiv:hep-th/0611272]. 
[12] J. Casalderrey-Solana and D. Teaney, "Heavy quark diffusion in strongly coupled $N=4$ Yang Mills," Phys. Rev. D 74 (2006) 085012 [ArXiv:hep-ph/0605199].

[13] J. Polchinski and M. J. Strassler, "Hard scattering and gauge/string duality," Phys. Rev. Lett. 88 (2002) 031601 [ArXiv:hep-th/0109174].

[14] U. Gursoy and E. Kiritsis, "Exploring improved holographic theories for QCD: Part I," JHEP 0802 (2008) 032 [ArXiv:0707.1324][hep-th];

U. Gursoy, E. Kiritsis and F. Nitti, "Exploring improved holographic theories for QCD: Part II," JHEP 0802 (2008) 019 [ArXiv:0707.1349] [hep-th].

[15] E. Kiritsis, "Dissecting the string theory dual of QCD," [ArXiv:0901.1772][hep-th].

[16] S. S. Gubser and A. Nellore, "Mimicking the QCD equation of state with a dual black hole," [ArXiv:0804.0434] [hep-th].

[17] R. Casero, E. Kiritsis and A. Paredes, "Chiral symmetry breaking as open string tachyon condensation," [ArXiv:hep-th/0702155.

[18] U. Gursoy, E. Kiritsis, L. Mazzanti and F. Nitti, "Deconfinement and Gluon Plasma Dynamics in Improved Holographic QCD, Phys. Rev. Lett. 101, 181601 (2008) [ArXiv:0804.0899][hep-th].

[19] U. Gursoy, E. Kiritsis, L. Mazzanti and F. Nitti, "Holography and Thermodynamics of 5D Dilaton-gravity, [ArXiv:0812.0792][hep-th].

[20] O. DeWolfe and C. Rosen, "Robustness of Sound Speed and Jet Quenching for Gauge/Gravity Models of Hot QCD," [ArXiv:0903.1458][hep-th].

$[21]$ E. Vicari and H. Panagopoulos, "Theta dependence of $S U(N)$ gauge theories in the presence of a topological term," [ArXiv:0803.1593][hep-th].

[22] U. Gursoy, E. Kiritsis, L. Mazzanti and F. Nitti, "Improved Holographic Yang-Mills at Finite Temperature: Comparison with Data," Nucl. Phys. B 820 (2009) 148 [ArXiv:0903.2859][hep-th].

[23] P. Kraus, "Dynamics of anti-de Sitter domain walls," JHEP 9912 (1999) 011 [ArXiv:hep-th/9910149].

[24] E. Kiritsis, "Supergravity, D-brane probes and thermal super Yang-Mills: A comparison," JHEP 9910 (1999) 010 [ArXiv:hep-th/9906206].

[25] A. Kehagias and E. Kiritsis, "Mirage cosmology," JHEP 9911 (1999) 022 [arXiv:hep-th/9910174].

[26] E. Kiritsis, "Holography and brane-bulk energy exchange," JCAP 0510 (2005) 014 [ArXiv:hep-th/0504219].

[27] R. Baier, P. Romatschke, D. T. Son, A. O. Starinets and M. A. Stephanov, "Relativistic viscous hydrodynamics, conformal invariance, and holography,” JHEP 0804 (2008) 100 [ArXiv:0712.2451][hep-th]; S. Bhattacharyya, V. E. Hubeny, S. Minwalla and M. Rangamani, "Nonlinear Fluid Dynamics from Gravity," JHEP 0802 (2008) 045 [ArXiv:0712.2456][hep-th].

[28] H. B. Meyer, "A calculation of the shear viscosity in SU(3) gluodynamics," Phys. Rev. D 76 (2007) 101701 [ArXiv:0704.1801][hep-lat]. 
[29] D. Kharzeev and K. Tuchin, "Bulk viscosity of QCD matter near the critical temperature," JHEP 0809 (2008) 093 [ArXiv:0705.4280][hep-ph].

[30] F. Karsch, D. Kharzeev and K. Tuchin, "Universal properties of bulk viscosity near the QCD phase transition," Phys. Lett. B 663 (2008) 217 [ArXiv:0711.0914][hep-ph].

[31] D. Teaney, "Finite temperature spectral densities of momentum and R-charge correlators in $N=4$ Yang Mills theory," Phys. Rev. D 74, 045025 (2006) [ArXiv:hep-ph/0602044;

P. Romatschke and D. T. Son, "Spectral sum rules for the quark-gluon plasma," [ArXiv:0903.3946][hep-ph].

[32] H. B. Meyer, "A calculation of the bulk viscosity in SU(3) gluodynamics," Phys. Rev. Lett. 100 (2008) 162001 [ArXiv:0710.3717][hep-lat].

[33] U. Heinz, talk at the "The Extra Strong Quark Gluon Plasma (ESQGP)", Stony Brook, 2008

[34] A. Adare et al. [PHENIX Collaboration], "Energy Loss and Flow of Heavy Quarks in Au $+A u$ Collisions at $\sqrt{s_{N N}}=200$ GeV," Phys. Rev. Lett. 98 (2007) 172301 [ArXiv:nucl-ex/0611018].

[35] N. Borghini and U. A. Wiedemann, "Predictions for the LHC heavy ion programme," J. Phys. G 35 (2008) 023001 [ArXiv:0707.0564][hep-ph].

[36] R. Baier, Y. L. Dokshitzer, A. H. Mueller, S. Peigne and D. Schiff, "Radiative energy loss of high energy quarks and gluons in a finite-volume quark-gluon plasma," Nucl. Phys. B 483 (1997) 291 [ArXiv:hep-ph/9607355];

"Radiative energy loss and $p($ T)-broadening of high energy partons in nuclei," Nucl. Phys. B 484 (1997) 265 [ArXiv:hep-ph/9608322];

B. G. Zakharov, "Radiative energy loss of high energy quarks in finite-size nuclear matter and quark-gluon plasma," JETP Lett. 65 (1997) 615 [ArXiv:hep-ph/9704255].

[37] Y. Akamatsu, T. Hatsuda and T. Hirano, "Heavy Quark Diffusion with Relativistic Langevin Dynamics in the Quark-Gluon Fluid," [ArXiv:0809.1499][hep-ph].

[38] S. S. Gubser, S. S. Pufu, F. D. Rocha and A. Yarom, "Energy loss in a strongly coupled thermal medium and the gauge-string duality," [ArXiv:0902.4041][hep-th].

[39] H. Liu, K. Rajagopal and U. A. Wiedemann, "Calculating the jet quenching parameter from AdS/CFT," Phys. Rev. Lett. 97 (2006) 182301 [ArXiv:hep-ph/0605178].

[40] H. Liu, K. Rajagopal and U. A. Wiedemann, "Wilson loops in heavy ion collisions and their calculation in AdS/CFT," JHEP 0703 (2007) 066; [ArXiv:hep-ph/0612168.

[41] B. Svetitsky, "Diffusion of Charmed Quark in the Quark Gluon PLasma," Phys. Rev. D 37 (1988) 2484.

[42] H. van Hees and R. Rapp, "Thermalization of Heavy Quarks in the Quark-Gluon Plasma," Phys. Rev. C 71 (2005) 034907 [ArXiv:nucl-th/0412015].

[43] G. D. Moore and D. Teaney, "How much do heavy quarks thermalize in a heavy ion collision?," Phys. Rev. C 71 (2005) 064904 [ArXiv:hep-ph/0412346].

[44] H. van Hees, V. Greco and R. Rapp, "Heavy-quark probes of the quark-gluon plasma at RHIC," Phys. Rev. C 73 (2006) 034913 [ArXiv:nucl-th/0508055].

[45] S. S. Gubser, "Momentum fluctuations of heavy quarks in the gauge-string duality," Nucl. Phys. B 790 (2008) 175 [ArXiv:hep-th/0612143. 
[46] J. de Boer, V. E. Hubeny, M. Rangamani and M. Shigemori, "Brownian motion in AdS/CFT," [ArXiv:0812.5112][hep-th].

[47] D. T. Son and D. Teaney, "Thermal Noise and Stochastic Strings in AdS/CFT," [ArXiv:0901.2338][hep-th].

[48] G. C. Giecold, E. Iancu and A. H. Mueller, "Stochastic trailing string and Langevin dynamics from AdS/CFT," [ArXiv:0903.1840][hep-th].

[49] F. Debbasch, K. Mallick and K. P. Rivet, J. of Stat. Phys., 88 (1997) 945.

C. Chevalier and F. Debbasch, J. Math. Phys. 49 (2008) 043303.

[50] S. Caron-Huot, P. Kovtun, G. D. Moore, A. Starinets and L. G. Yaffe, "Photon and dilepton production in supersymmetric Yang-Mills plasma," JHEP 0612 (2006) 015 [ArXiv:hep-th/0607237].

[51] S. J. Sin and I. Zahed, "Holography of radiation and jet quenching," Phys. Lett. B 608 (2005) 265 [ArXiv:hep-th/0407215];

"Ampere's Law and Energy Loss in AdS/CFT Duality," Phys. Lett. B 648 (2007) 318 [ArXiv:hep-ph/0606049].

[52] E. Witten, "Anti-de Sitter space, thermal phase transition, and confinement in gauge theories," Adv. Theor. Math. Phys. 2 (1998) 505 [ArXiv:hep-th/9803131];

[53] Y. Kinar, E. Schreiber and J. Sonnenschein, "Q anti-Q potential from strings in curved spacetime: Classical results," Nucl. Phys. B 566, 103 (2000) [ArXiv:hep-th/9811192].

[54] J. Heitger, H. Simma, R. Sommer and U. Wolff [ALPHA collaboration], "The Schroedinger functional coupling in quenched QCD at low energies," Nucl. Phys. Proc. Suppl. 106 (2002) 859 [ArXiv:hep-lat/0110201].

[55] J. Alanen, K. Kajantie and V. Suur-Uski, "A gauge/gravity duality model for gauge theory thermodynamics," Phys. Rev. D 80 (2009) 126008 [ArXiv:0911.2114][hep-ph].

[56] G. Boyd, J. Engels, F. Karsch, E. Laermann, C. Legeland, M. Lutgemeier and B. Petersson, "Thermodynamics of SU(3) Lattice Gauge Theory," Nucl. Phys. B 469, 419 (1996) [ArXiv:hep-lat/9602007].

[57] B. Lucini, M. Teper and U. Wenger, "The deconfinement transition in $S U(N)$ gauge theories," Phys. Lett. B 545 (2002) 197 [ArXiv:hep-lat/0206029];

B. Lucini, M. Teper and U. Wenger, "The high temperature phase transition in $S U(N)$ gauge theories," JHEP 0401 (2004) 061 [ArXiv:hep-lat/0307017]. B. Bringoltz and M. Teper, "The pressure of the $S U(N)$ lattice gauge theory at large- $N$," Phys. Lett. B 628, 113 (2005) [ArXiv:hep-lat/0506034.

[58] M. Panero, "Thermodynamics of the QCD plasma and the large-N limit," Phys. Rev. Lett. 103 (2009) 232001 [ArXiv:0907.3719][hep-lat]; "Thermodynamics of the strongly interacting gluon plasma in the large- $N$ limit," [ArXiv:0912.2448][hep-lat].

[59] B. Lucini, M. Teper and U. Wenger, "Properties of the deconfining phase transition in $S U(N)$ gauge theories," JHEP 0502, 033 (2005) [ArXiv:hep-lat/0502003].

[60] E. Kiritsis and F. Nitti, "On massless $4 D$ Gravitons from 5D Asymptotically AdS Space-times," Nucl. Phys. B 772 (2007) 67 [ArXiv:hep-th/0611344]. 
[61] C. J. Morningstar and M. J. Peardon, "The glueball spectrum from an anisotropic lattice study," Phys. Rev. D 60, 034509 (1999) [ArXiv:hep-lat/9901004];

Y. Chen et al., "Glueball spectrum and matrix elements on anisotropic lattices," Phys. Rev. D 73, 014516 (2006) [ArXiv:hep-lat/0510074].

[62] B. Lucini and M. Teper, "SU(N) gauge theories in four dimensions: Exploring the approach to $N=\infty$," JHEP 0106, 050 (2001) [ArXiv:hep-lat/0103027].

[63] H. B. Meyer, "Glueball Regge trajectories," [ArXiv:hep-lat/0508002].

[64] M. Cheng et al., "The Spatial String Tension and Dimensional Reduction in QCD," Phys. Rev. D 78 (2008) 034506 [ArXiv:0806.3264][hep-lat].

[65] Y. Schroder and M. Laine, "Spatial string tension revisited," PoS LAT2005 (2006) 180 ArXiv:hep-lat/0509104/.

[66] J. Alanen, K. Kajantie and V. Suur-Uski, "Spatial string tension of finite temperature QCD matter in gauge/gravity duality," Phys. Rev. D 80 (2009) 075017 [ArXiv:0905.2032][hep-ph].

[67] D. f. Zeng, "Heavy quark potentials in some renormalization group revised AdS/QCD models," Phys. Rev. D 78 (2008) 126006 [ArXiv:0805.2733][hep-th].

[68] B. Galow, E. Megias, J. Nian and H. J. Pirner, "Phenomenology of AdS/QCD and Its Gravity Dual," [ArXiv:0911.0627][hep-ph].

[69] J. Noronha, "Connecting Polyakov Loops to the Thermodynamics of $S U\left(N_{c}\right)$ Gauge Theories Using the Gauge-String Duality," [ArXiv:0910.1261][hep-th]; "Polyakov Loops in Strongly-Coupled Plasmas with Gravity Duals," [ArXiv:1001.3155][hep-th].

[70] L. Del Debbio, L. Giusti and C. Pica, "Topological susceptibility in the SU(3) gauge theory," Phys. Rev. Lett. 94, 032003 (2005) [ArXiv:hep-th/0407052].

[71] G. D. Moore and O. Saremi, "Bulk viscosity and spectral functions in QCD," JHEP 0809 (2008) 015 [ArXiv:0805.4201][hep-ph].

[72] G. Policastro, D. T. Son and A. O. Starinets, "The shear viscosity of strongly coupled $N=4$ supersymmetric Yang-Mills plasma," Phys. Rev. Lett. 87 (2001) 081601 [ArXiv:hep-th/0104066].

[73] T. Springer, "Sound Mode Hydrodynamics from Bulk Scalar Fields," Phys. Rev. D 79, 046003 (2009) [ArXiv:arXiv:0810.4354][hep-th].

[74] S. S. Gubser, S. S. Pufu and F. D. Rocha, "Bulk viscosity of strongly coupled plasmas with holographic duals," JHEP 0808 (2008) 085 [ArXiv:0806.0407][hep-th].

[75] P. Arnold, C. Dogan and G. D. Moore, "The bulk viscosity of high-temperature QCD," Phys. Rev. D 74 (2006) 085021 [arXiv:hep-ph/0608012].

[76] U. Gursoy, E. Kiritsis, G. Michalogiorgakis and F. Nitti, "Thermal Transport and Drag Force in Improved Holographic QCD," JHEP 0912 (2009) 056 [ArXiv:0906.1890][hep-ph].

[77] H. A. Chamblin and H. S. Reall, "Dynamic dilatonic domain walls," Nucl. Phys. B 562 (1999) 133 [arXiv:hep-th/9903225].

[78] A. Buchel, "Bulk viscosity of gauge theory plasma at strong coupling," Phys. Lett. B 663 (2008) 286 [ArXiv:0708.3459][hep-th]. 
[79] I. Kanitscheider and K. Skenderis, "Universal hydrodynamics of non-conformal branes," JHEP 0904 (2009) 062 [ArXiv:0901.1487][hep-th].

[80] H. Song and U. W. Heinz, "Extracting the QGP viscosity from RHIC data - a status report from viscous hydrodynamics," [ArXiv:0812.4274][nucl-th].

[81] A. Yarom, "Notes on the bulk viscosity of holographic gauge theory plasmas," [ArXiv:0912.2100][hep-th].

[82] A. Cherman, T. D. Cohen and A. Nellore, "A bound on the speed of sound from holography," Phys. Rev. D 80 (2009) 066003 [ArXiv:0905.0903][hep-th];

A. Cherman and A. Nellore, "Universal relations of transport coefficients from holography," Phys. Rev. D 80 (2009) 066006 [ArXiv:0905.2969][hep-th].

[83] P. M. Hohler and M. A. Stephanov, "Holography and the speed of sound at high temperatures," Phys. Rev. D 80 (2009) 066002 [ArXiv:0905.0900][hep-th].

[84] G. Beuf, C. Marquet and B. W. Xiao, "Heavy-quark energy loss and thermalization in a strongly coupled SYM plasma, [ArXiv:0812.1051][hep-ph].

[85] S. Datta, F. Karsch, P. Petreczky and I. Wetzorke, "Behavior of charmonium systems after deconfinement," Phys. Rev. D 69, 094507 (2004); [ArXiv:hep-lat/0312037].

[86] M. Luzum and P. Romatschke, "Viscous Hydrodynamic Predictions for Nuclear Collisions at the LHC," [ArXiv:arXiv:0901.4588][nucl-th].

[87] C. P. Herzog, "A holographic prediction of the deconfinement temperature," Phys. Rev. Lett. 98, 091601 (2007) [ArXiv:hep-th/0608151.

[88] C. A. Ballon Bayona, H. Boschi-Filho, N. R. F. Braga and L. A. Pando Zayas, Phys. Rev. D 77, 046002 (2008) [ArXiv:0705.1529][hep-th].

[89] O. Andreev, "Some Thermodynamic Aspects of Pure Glue, Fuzzy Bags and Gauge/String Duality," Phys. Rev. D 76 (2007) 087702 [ArXiv:0706.3120] [hep-ph].

[90] K. Kajantie, T. Tahkokallio and J. T. Yee, “Thermodynamics of AdS/QCD," JHEP 0701 (2007) 019 [ArXiv:hep-ph/0609254].

[91] U. Gursoy, E. Kiritsis, L. Mazzanti and F. Nitti, "Langevin diffusion of heavy quarks in non-conformal holographic backgrounds," [ArXiv:1006.3261][hep-th]. 